\title{
An Itô Formula for rough partial differential equations and some applications
}

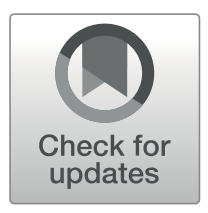

\author{
Antoine Hocquet $^{1}$ (D) Torstein Nilssen $^{2}$
}

Received: 6 February 2019 / Accepted: 24 January 2020 / Published online: 20 April 2020

(C) The Author(s) 2020

\begin{abstract}
We investigate existence, uniqueness and regularity for solutions of rough parabolic equations of the form $\partial_{t} u-A_{t} u-f=\left(\dot{X}_{t}(x) \cdot \nabla+\dot{Y}_{t}(x)\right) u$ on $[0, T] \times \mathbb{R}^{d}$. To do so, we introduce a concept of "differential rough driver", which comes with a counterpart of the usual controlled paths spaces in rough paths theory, built on the Sobolev spaces $W^{k, p}$. We also define a natural notion of geometricity in this context, and show how it relates to a product formula for controlled paths. In the case of transport noise (i.e. when $Y=0$ ), we use this framework to prove an Itô Formula (in the sense of a chain rule) for Nemytskii operations of the form $u \mapsto F(u)$, where $F$ is $C^{2}$ and vanishes at the origin. Our method is based on energy estimates, and a generalization of the Moser Iteration argument to prove boundedness of a dense class of solutions of parabolic problems as above. In particular, we avoid the use of flow transformations and work directly at the level of the original equation. We also show the corresponding chain rule for $F(u)=|u|^{p}$ with $p \geq 2$, but also when $Y \neq 0$ and $p \geq 4$. As an application of these results, we prove existence and uniqueness of a suitable class of $L^{p}$-solutions of parabolic equations with multiplicative noise. Another related development is the homogeneous Dirichlet boundary problem on a smooth domain, for which a weak maximum principle is shown under appropriate assumptions on the coefficients.
\end{abstract}

Keywords Rough paths · Rough PDEs · Energy method · Weak solutions · Itô formula · Maximum principle $\cdot$ Renormalized solutions

Mathematics Subject Classification (2010) 60L50 $\cdot 60 \mathrm{H} 15 \cdot 35 \mathrm{~A} 15 \cdot 35 \mathrm{~B} 50 \cdot 35 \mathrm{D} 30$

Financial support by the DFG via Research Unit FOR 2402 is gratefully acknowledged.

Antoine Hocquet

antoine.hocquet@wanadoo.fr

Torstein Nilssen

torstein.nilssen@uia.no

1 Institut für Mathematik, Technische Universität Berlin,

Straße des 17. Juni 136, D-10623, Berlin, Germany

2 Mathematical Institute, University of Agder, Universitetsveien 25, 4630 Kristiansand, Norway 


\section{Introduction}

Motivations Consider a stochastic partial differential equation with multiplicative noise of the form

$$
\mathrm{d} u_{t}-\Delta u_{t} \mathrm{~d} t=\partial_{i} u_{t} \mathrm{~d} X_{t}^{i}(x)+u_{t} \mathrm{~d} X_{t}^{0}(x), \quad \text { on } \quad(0, T] \times \mathbb{R}^{d}
$$

where $\partial_{i}=\frac{\partial}{\partial x_{i}}, T \in(0, \infty)$ denotes a fixed time horizon, $\left(X^{i}\right)_{i=0, \ldots, d}$ denotes some $Q$-Wiener process (sufficiently smooth in $x$ ), and throughout the paper we use Einstein's summation convention over repeated indices. For now the product with the above differentials is subject to different possible meanings (for instance Stratonovitch or Itô).

Equations such as (1.1) arise in a number of different stochastic models. To name a few, this s filtering theory [34], McKean-Vlasov equations [41], or pathwise stochastic control problems (see for instance [11, Example 2] and references therein). In the more general context of a degenerate left hand side, this type of noise appears in stochastic transport equations (with $X^{0}=0$ ), where a regularization by noise phenomenon is observed [12, 22, $52,54]$, or in stochastic conservation laws, see [33] for an overview. We also mention the works $[9,15]$ where the authors solve an equation similar to $(1.1)$, with the difference that they consider a vector field $X_{t}^{i}(x)$ which is rough with respect to the space-like variable.

The way (1.1) is usually dealt with is by definition of an appropriate functional setting, in which standard Itô calculus tools can be used. We refer for instance to the classical works of Pardoux, Krylov and Rozovskii [45, 56]. Although these approaches are quite sucessful, it is well-known that the solution map $X \mapsto u$ is not continuous in general. This constitutes an important motivation for introducing a rough paths formulation of (1.1) (in particular because the examples given above display a need for stability results, see [26]). Rough parabolic differential equations such as (1.1) have been investigated in $[10,11,24,26]$ where a viscosity formulation is proposed, based on ideas of Lions and Souganidis [48, 49]. Despite their success, these papers appeal to an extensive use of flow transformation techniques, which has some conceptual disadvantages. In particular, they have to make the assumption that the solutions are obtained as limits of approximations. To the best of our knowledge, the Feynmann-Kac representation technique used in [17], constitutes the first attempt to deal with (1.1) directly (there is also the semigroup approach of Gubinelli, Deya and Tindel [18, 31], but their results do not seem to cover the case of a gradient noise as above).

One of our main purposes in this paper is to pursue the variational approach initiated by Deya, Gubinelli, Hofmanová and Tindel in [19], by defining, among other things, a suitable functional setting for generalized versions of (1.1). In this sense, we will particularly emphasize the topological aspects associated with (1.1), for instance by introducing the controlled paths spaces $\mathcal{D}_{B}^{\alpha, p}$, as well as their parabolic counterpart $\mathcal{H}_{B}^{\alpha, p}$ (see sections 3 and 4). Working with classical PDE techniques such as energy estimates and maximum principles, our contribution can be seen as an attempt to extend Krylov's analytic approach [42] to the RPDE context. One of the key concepts we will use here is that of an unbounded rough driver, as introduced by Bailleul and Gubinelli in [4]. More specifically, we will introduce a notion of differential rough driver, which is a particular case of the former (see Definition 2.1). We will also provide a natural, intrinsic notion of geometricity for differential rough drivers. As shown in Lemma 2.1, geometric differential rough drivers display remarkable algebraic properties. In particular, they are simultaneously symmetric, closed and renormalizable in the sense of [4, definitions 5.3, $5.4 \& 5.7]$. In contrast with the previous works $[19,38,39]$, we will be able to consider these objects "as such", in the sense that we will not refer to any (geometric) finite-dimensional rough path. This observation, which can be 
seen as one of our main contributions, allows us to gain generality in the statements and, hopefully, to improve the clarity of the presentation.

The importance of geometricity and its relation to stochastic parabolicity In contrast with the recent developments on rough parabolic equations [3, 30, 31, 35-37, 55] (for results related to Itô Formula in this case, see $[6,60])$, the noise term in (1.1) is not singular with respect to the space-variable, so that in appearance (1.1) does not fall into the category of "singular PDEs". However, difficulties arise from the fact that for all times $t$ the operation $u \mapsto X_{t} \cdot \nabla u$ is unbounded. A side effect of this property is that the low time-regularity of solutions implies in turn low space-regularity, as can be seen by the scaling properties of the equation. In the case of $X=W$ being a Brownian motion and $X^{0}=0$, it is easily seen that for $\varepsilon>0$ the transform $(t, x) \rightarrow\left(\varepsilon^{2} t, \varepsilon x\right)$ leaves the equation invariant (using the scaling properties of $W$ ). Leaving aside mathematical rigor, this type of invariance indicates that (1.1) cannot be considered as a perturbation of a heat equation at small scales. In this sense, the (1.1) is not really parabolic and the use of semigroups and variation of constants formulae is inoperative (we nevertheless refer to the recent works [28, 29] in a similar but "subcritical" context). The situation can go even worse if $X=W^{H}$ is a fractional Brownian motion with hurst index $1 / 3<H<1 / 2$, a case that is covered by our results. In this case, the transport term $\partial_{t}-\dot{W}_{t}^{H} \cdot \nabla$ dominates, even though the drift term has two spatial derivatives. This might be a loose explanation why some of the arguments below seem to have a transport flavour (the bounds (4.18) which are needed in the tensorization argument of Section 4 can be understood as a "commutator lemma" à la Di Perna Lions [20]; see Appendix A.2). As a matter of fact, the fractional Brownian case enters the category of "supercritical" equations in the sense of [36, Section 8], and this is so regardless of the space dimension $d$.

In this context, the assumption that $X$ is geometric turns out to be essential. To illustrate why, let us go back to the standard Brownian motion case, more precisely let $d=1$, consider $X_{t}=b W_{t}, b \in \mathbb{R}$ being a constant, and for simplicity take $X^{0}=0$. Assume for a moment that (1.1) is understood in the sense of Itô, so that the corresponding rough path formulation would violate geometricity. Computing formally the Itô Formula for the square of the $L^{2}$-norm of the solution, one sees that the correction term is given by $\int_{\mathbb{R}^{d}} b^{2}\left(\partial_{x} u\right)^{2}$, which dangerously competes with the conservative term $-2 \int_{\mathbb{R}^{d}}\left(\partial_{x} u\right)^{2}$ brought by the Laplacian. In particular, the usual technique to obtain an a priori estimate for $u$ fails unless $1 / 2 b^{2}<1$, which is a condition known as strong parabolicity. This assumption is in fact necessary to ensure well-posedness as can be seen by taking the spatial Fourier transform in the equation (we refer the reader to [45, Section III.3]). If on the other hand (1.1) is understood in the Stratonovitch sense, the latter problem disappears, and this is to be related to the fact that a Stratonovitch equation satisfies a "standard" chain rule of the form

$$
\mathrm{d}(F(u))=F^{\prime}(u) \circ \mathrm{d} u
$$

(meaning in particular that no correction term of the previous form appears). Besides introducing a new functional framework for (1.1), our main objective in this paper is to investigate the chain rule (1.2), which will be systematically addressed in the transport-noise case, assuming "geometricity of the driving noise" (understood at the level of the differential rough driver, see Definition 2.2). In the stochastic setting, the geometricity assumption essentially means that the iterated integrals which define the second level $\mathbb{L}_{t}$ of $\mathbf{X}_{t}$ should be understood in the Stratonovitch sense. Nevertheless, we point out that (1.1) can always be translated in terms of an equivalent Stratonovitch equation. If strong parabolicity is 
assumed, it is straightforward to check that the corrected equation has still the parabolic form (1.3), and hence our main results still apply in this practical case.

Settings and summary of the results In this paper, we interpret (1.1) as the rough equation

$$
\left\{\begin{array}{l}
\mathrm{d} u_{t}-\left(A_{t} u+f_{t}(x)\right) \mathrm{d} t=\mathrm{d} \mathbf{B}_{t} u_{t}, \quad \text { on }(0, T] \times \mathbb{R}^{d} \\
u_{0} \text { given in } L^{p}\left(\mathbb{R}^{d}\right),
\end{array}\right.
$$

where the unknown $u_{t}(x)$ is seen as a path with values in the Lebesgue space $L^{p}\left(\mathbb{R}^{d}\right)$, for some $p \in[1, \infty]$. Here

$$
\mathbf{B}=\left(B^{1}, B^{2}\right)
$$

denotes some kind of two-step "enhancement" of the time-dependent family of differential operators

$$
B_{t}=B_{0 t}^{1}:=X_{t}^{i}(x) \partial_{i}+X_{t}^{0}(x), \quad t \in[0, T],
$$

for $\left(X_{t}^{i}(x)\right)_{0 \leq i \leq d}$ sufficiently regular in space. From the point of view of the coefficient path, it will be seen that $\left(t \mapsto X_{t}(x)\right)$ must be accompanied with an additional object

$$
\mathbb{L}_{s t}^{i}(x), \quad i=0, \ldots d, \quad 0 \leq s \leq t \leq T, \quad x \in \mathbb{R}^{d},
$$

akin to the usual Lévy area for two-step geometric rough paths with real-valued coordinates. The knowledge of $\mathbb{L}^{i}$ is necessary (and sufficient) to give a proper meaning for (1.3). As will be seen in the manuscript, it is heuristically filling the gaps in order to make sense of the (a priori ill-defined) iterated integral

$$
\begin{aligned}
B_{s t}^{2} & =\iint_{s<r_{1}<r_{2}<t} \mathrm{~d} B_{r_{2}} \circ \mathrm{d} B_{r_{1}} \\
& :=\frac{1}{2} X_{s t}^{i} X_{s t}^{j} \partial_{i j}+\left(\mathbb{L}_{s t}^{i}+X_{s t}^{i} X_{s t}^{0}\right) \partial_{i}+\mathbb{L}_{s t}^{0}+\frac{1}{2}\left(X_{s t}^{0}\right)^{2}, \\
& \text { for } \quad 0 \leq s \leq t \leq T \quad \text { and } \quad x \in \mathbb{R}^{d},
\end{aligned}
$$

where 'o' denotes the composition of linear operators. In particular, there is a one-to-one correspondence between $\mathbf{B}$ and the enhancement $(X, \mathbb{L})$ of its coefficient path. Throughout the paper, the pair $\mathbf{X}=(X, \mathbb{L})$ is therefore considered as part of the data, and so is $\mathbf{B}$ through (1.5). For simplicity, the path $X$ will be assumed to have bounded $q$-variation with $q=\frac{1}{\alpha}$ (including the $\alpha$-Hölder case), for some $\alpha>1 / 3$. It will be sometimes more convenient to rewrite (1.3) under the following form

$$
\left\{\begin{array}{l}
\mathrm{d} u-\left(A_{t} u+f_{t}\right) \mathrm{d} t=\left(\mathrm{d} \mathbf{X} \cdot \nabla+\mathrm{d} \mathbf{X}^{0}\right) u_{t} \quad \text { on }(0, T] \times \mathbb{R}^{d} \\
u_{0} \in L^{p}
\end{array}\right.
$$

which has the advantage of being more explicit.

In keeping with Gubinelli's approach [32], the integration map which is implicitly associated with the right hand side of (1.3), only makes sense on a set of paths $u:[0, T] \rightarrow L^{p}$ that are controlled by $B$, a notion that will be introduced in Section 2.3. Concerning the left hand side of (2.19), we will assume throughout the paper that $A_{t}$ is a time-dependent family of elliptic operators on divergence form

$$
A_{t} u(x)=\partial_{i}\left(a^{i j}(t, x) \partial_{j} u(x)\right),
$$

whith coefficients $a^{i j}$ being possibly discontinuous but bounded above and below (see assumption 2.1). Correspondingly, the free term $f$ will be an element of the Sobolev space 
$L^{2}\left(0, T ; H^{-1}\right)$. Our first main achievement is to prove well-posedness for (1.3), for a class of controlled paths $u:[0, T] \rightarrow L^{2}\left(\mathbb{R}^{d}\right)$ having finite energy

$$
\sup _{t \in[0, T]}\left|u_{t}\right|_{L^{2}}^{2}+\int_{0}^{T}\left|\nabla u_{t}\right|_{L^{2}}^{2} \mathrm{~d} t<\infty,
$$

in the case where $\mathbf{B}$ is geometric. This will be stated in Theorem 2.1, completing the results of [38].

Next, we will address the problem of writing an Itô formula for solutions of (1.3), where in addition of geometricity, we will assume that $\mathbf{B}$ is "transport-like", that is:

$$
X^{0}=0 \quad \text { in (1.4). }
$$

The problem of writing a chain rule for (1.3) arises in a very natural way when studying the well-posedness of (1.1), as illustrated by the previous paragraph and the search for an energy estimate (this corresponds to the choice $F(z)=z^{2}$ in (1.2)). The justification of the chain rule is also useful to establish comparison principles, where the corresponding choice of function would be for instance $F(z)=z^{ \pm}$, or a suitable regularized version thereof. Under the assumption (1.7), we will prove that a chain rule like (1.2) holds for any $F \in C^{2}(\mathbb{R}, \mathbb{R})$ with $F(0)=F^{\prime}(0)=0$ and $\left|F^{\prime \prime}\right|_{L^{\infty}}<\infty$. Concretely, we will see that

$$
\mathrm{d}(F(u))-F^{\prime}(u)\left(A_{t} u+f\right) \mathrm{d} t=\mathrm{d} \mathbf{X} \cdot \nabla(F(u))
$$

(see Theorem 2.2 for a precise statement). The formula (1.8) will be applied in particular to obtain a weak maximum principle for an appropriate subclass of problems of the form (1.3), as will be stated in Theorem 2.5. We insist on the fact that, because of the lack of spaceregularity of solutions, (1.8) is not a trivial statement. In particular, the solution $u$ fails in general to satisfy the hypotheses of [23, Proposition 7.6], see Remark 2.4. Note that in some sense, (1.8) can be seen as a parabolic analogue to the renormalization property for transport equations in Sobolev spaces [2, 16, 20]. Roughly speaking, renormalized solutions could be defined as elements $u$ of the controlled path space so that (1.8) holds for any $F$ as above; hence (1.8) shows that solutions of finite energy are renormalized. On the other hand, if $u$ is renormalized, taking $F=(\cdot)^{2}$ will show that $u$ is itself an $L^{2}$-solution, and hence (1.8) can be understood as the statement that the two notions are equivalent.

Regarding applications, the chain rule for the $L^{p}$-norm of solutions $u:[0, T] \rightarrow L^{p}$ (that is (1.8) with $F(z)=|z|^{p}$ ) is of particular interest for SPDE purposes. In the stochastic setting, this echoes the works of Krylov and Kim for stochastic equations in $L^{p}$ spaces [40, $43,44]$, where the corresponding Itô Formula is an essential tool. In this paper, we will investigate the analogue for rough paths, that is for every $L^{p}$-solution $u$ of (1.3), and under some mild assumptions on $f$ and $u_{0}$, we will see that

$$
\mathrm{d}|u|^{p}-p u|u|^{p-2}\left(A_{t} u+f\right) \mathrm{d} t=\left(\mathrm{d} \mathbf{X} \cdot \nabla+p \mathbf{X}^{0}\right)|u|^{p}
$$

as long as $p \geq 4$ (this can be relaxed to $p \geq 2$ when $X^{0}=0$ ). We note that, since $F^{\prime \prime}$ is not bounded, (1.9) is not a simple consequence of (1.8), even when the multiplicative part is zero. Nevertheless, using rough paths stability results that come for free with our formulation, it will be seen that (1.9) admits a relatively simple proof. In our way to prove this formula, we shall also address existence and uniqueness for a suitable class of $L^{p}$ solutions of parabolic equations with multiplicative noise.

Due to the relative length of this paper, and since this drastically complicates the algebra, we chose to postpone the treatment of a more general Itô Formula (taking for instance $X^{0} \neq$ 0 in (1.4), or even a non-geometric B) in a future work. Similarly, we could have considered 
an additional rough input of additive form. More general operators $A$ (for instance adding a perturbation $b^{i}(t, x) \partial_{i} u+c(t, x) u$ with integrability conditions on $b, c$, see [38]) and more general boundary problems, could also be investigated following the same ideas, but for the sake of simplicity we restrain from doing so.

Organization of the paper Our main results concerning existence, uniqueness, stability and the chain rule for (1.3), will be given in Section 2, where we also introduce notations and definitions. In particular, we introduce an intrinsic formulation of (1.3), in the spirit of [19]. We will complete our results by a criterion for boundedness of solutions, a chain rule for the $L^{p}$-norm of solutions, and a weak maximum principle for the Dirichlet problem on a bounded domain. In Section 3 we state some facts that will be used throughout the paper, such as the Sewing Lemma or the so-called "Rough Gronwall" argument (as stated in [19]). The main novelty of this section is that we introduce a notion of controlled path space $\mathcal{D}_{B}^{\alpha, p}$, with respect to a differential rough driver $\mathbf{B}$. We then state and re-prove the so-called "remainder estimates" as given by Deya, Gubinelli, Hofmanová and Tindel in [19, Theorem 2.5]. We provide an alternative formulation of this result, which has the conceptual advantage of being understood as an a priori estimate in $\mathcal{D}_{B}^{\alpha, p}$ (as in the usual finite-dimensional controlled path picture). In Section 4 , we define a suitable functional setting for rough parabolic equations by introducing the parabolic spaces $\mathcal{H}_{B}^{\alpha, p}$. We will then state one of the core arguments of this paper, which is the "product formula" (Proposition 4.1). By reiteration of the product, we will obtain the chain rule on monomials of any bounded solution, and on polynomials by linearity.

In Section 5, we use this result to solve a class of rough, non-degenerate parabolic equation with free terms in the space $L^{2}\left(H^{-1}\right)$. This is done via energy estimates, and the use of the Rough Gronwall Lemma. In Section 6 we show, using a Moser Iteration, that a "relatively large" class of solutions to rough parabolic problems of the form (1.3) is made of elements which are locally bounded. This observation, together with the fact that a chain rule holds for polynomials of a bounded solution, will then allow us to prove the claimed Itô formula in Section 7. The corresponding proof for the $L^{p}$-norm, as well as the solvability for an appropriate class of $L^{p}$-solutions, will be dealt with at the end of Section 7 . It is based on a different argument using approximation and stability results for rough partial differential equations.

Section 8 is devoted to the proof of Theorem 2.5. After proving the solvability of the homogeneous Dirichlet problem on a smooth, bounded domain, we show, using our Itô Formula, that the solutions satisfy a weak maximum principle.

In Appendix A, we shall give the proof of some technical facts verified by any geometric differential rough driver, generalizing [19, Section 3.2]. Finally, Appendix B will be devoted to a quick discussion on the uniqueness of the Gubinelli derivative, and on the "non-commutative brackets" $[\mathbf{B}]_{s t}=B_{s t}^{2}-\frac{1}{2} B_{s t}^{1} \circ B_{s t}^{1}$.

\section{Preliminaries and main results}

\subsection{Notation}

Throughout the paper, the notation $K \subset \subset \mathbb{R}^{d}$ stands for " $K$ is a compact set in $\mathbb{R}^{d}$ ". The symbol $T>0$ refers to a finite, fixed time-horizon.

By $\mathbb{N}$, we denote the set of natural integers $1,2, \ldots$, and we let $\mathbb{N}_{0}:=\mathbb{N} \cup\{0\}$, while $\mathbb{Z}:=\mathbb{N}_{0} \cup(-\mathbb{N})$. Real numbers are denoted by $\mathbb{R}$, and we let moreover $\mathbb{R}_{+}:=[0, \infty)$. 
Given Banach spaces $X, Y$, we will denote by $\mathscr{L}(X, Y)$ the space of linear, continuous maps from $X$ to $Y$, endowed with the operator norm. For $f$ in $X^{*}:=\mathscr{L}(X, \mathbb{R})$, we denote the dual pairing by

$$
X^{*}\langle f, g\rangle_{X}
$$

(i.e. the evaluation of $f$ at $g \in X$ ). When they are clear from the context, we will simply omit the underlying spaces and write $\langle f, g\rangle$ instead.

Sobolev spaces and scales For an open smooth domain $U \subset \mathbb{R}^{d}$, we will consider the usual Lebesgue and Sobolev spaces in the space-like variable: $L^{p}(U), W^{k, p}(U)$, for $(k, p) \in \mathbb{Z} \times(1, \infty]$ or $p=1$ and $k \in \mathbb{N}_{0}$, and we distinguish the case $p=2$ by writing $H^{k}(U):=W^{2, k}(U)$; the corresponding norms will be simply denoted by $|\cdot|_{L^{p}(U)},|\cdot|_{W^{k, p}(U)},|\cdot|_{H^{k}(U)}$. With the exception of Section 3.2, the notations $L^{p}, W^{k, p}$ and $H^{k}$ refer to the whole space scenario $U=\mathbb{R}^{d}$. These spaces have local (resp. weak) analogues $L_{\mathrm{loc}}^{p}, W_{\mathrm{loc}}^{k, p}, H_{\mathrm{loc}}^{k}$ (resp. $L_{\mathrm{w}}^{p}, W_{\mathrm{w}}^{k, p}, H_{\mathrm{w}}^{k}$ ) which are defined as usual. When $k$ is negative, we adopt the convention that $W^{k, 1}$ is the range of the linear operator

$$
\left(\mathbf{f}^{\gamma}\right) \in L^{p}\left(\mathbb{R}^{d} ; \mathbb{R}^{\sum_{|\gamma| \leq-k}|\gamma|}\right) \mapsto\left(\partial_{\gamma} \mathbf{f}^{\gamma}\right)|\gamma| \leq-k
$$

where $|\gamma|:=\gamma_{1}+\cdots+\gamma_{d}$, and the derivatives are understood in distributional sense. Correspondingly, the norm of $f \in W^{k, 1}$ is defined as the infimum of the $L^{1}$-norms of any possible antiderivative $\mathbf{f}^{\gamma}$ of $f$. Note that with this convention, $W^{k, 1}$ identifies only with a proper subspace of the dual $\left(W_{0}^{|k|, \infty}\right)^{*}$, however this is coherent with the case $p>1$ (see for instance [8]). If $U \subset \mathbb{R}^{d}$ is a domain whose boundary is smooth and if $p \in[1, \infty]$, we define the spaces $W_{0}^{k, p}$ as

$$
W_{0}^{k, p}(U):=\left\{f \in W^{k, p} \text { s.t. }(v \cdot \nabla)^{j} f=0 \text { for } j \in \mathbb{N}_{0}, j<k-1 / p\right\} .
$$

where $v$ denotes the outward unit vector associated to $\partial U$.

In the sequel, we call a scale any graded family of topological vector spaces of the form $\left(E_{k},|\cdot|_{k}\right)_{k \in I}$ with $I \subset \mathbb{Z}$ such that $E_{k}$ is continuously embedded into $E_{k-1}$, for each $k \in I$. Note that, in the paper the set $I:=\{-3,-2,-1,0,1,2,3\}$ will be sufficient for our purposes.

For $0 \leq s \leq t \leq T$ and $f=f_{r}(x)$ we use the notation

$$
\|f\|_{L^{r}\left(s, t ; L^{q}\right)}:=\left(\int_{s}^{t}\left(\int_{\mathbb{R}^{d}}\left|f_{\tau}(x)\right|^{q} \mathrm{~d} x\right)^{r / q} \mathrm{~d} \tau\right)^{1 / r},
$$

and for simplicity we will sometimes write $\|f\|_{L^{r}\left(L^{q}\right)}$ as a shorthand for $\|f\|_{L^{r}\left(0, T ; L^{q}\right)}$. Furthermore, the space of continuous functions with values in a Fréchet space $E$ will be denoted by $C(0, T ; E)$. It is itself a Fréchet space, equipped with the family of semi-norms $\|f\|_{C(0, T ; E), \gamma}:=\sup _{r \in I} \gamma\left(f_{r}\right)$, for any semi-norm $\gamma$ of $E$.

Controls and $p$-variation spaces We will denote by $\Delta, \Delta_{2}$ the simplices

$$
\begin{aligned}
& \Delta:=\left\{(s, t) \in[0, T]^{2}, s \leq t\right\}, \\
& \Delta_{2}:=\left\{(s, \theta, t) \in[0, T]^{3}, s \leq \theta \leq t\right\} .
\end{aligned}
$$

If $E$ is a vector space and $g:[0, T] \rightarrow E$, we define a two-parameter element $\delta g$ as

$$
\delta g_{s t}:=g_{t}-g_{s}, \quad \text { for }(s, t) \in \Delta .
$$


Similarly, we define another operation $\tilde{\delta}$ by letting, for any $g: \Delta \rightarrow E, \tilde{\delta} g$ be the quantity

$$
\tilde{\delta} g_{s \theta t}:=g_{s t}-g_{s \theta}-g_{\theta t}, \quad \text { for }(s, \theta, t) \in \Delta_{2},
$$

and we recall that $\operatorname{Ker} \tilde{\delta}=\operatorname{Im} \delta$. As usual in the framework of controlled paths, we will omit the symbol $\sim$ on the second operation, and write $\delta$ instead of $\tilde{\delta}$.

We call control on $[0, T]$ any continuous, superadditive map $\omega: \Delta \rightarrow \mathbb{R}_{+}$, namely $\omega$ is such that for all $(s, \theta, t) \in \Delta_{2}$

$$
\omega(s, \theta)+\omega(\theta, t) \leq \omega(s, t)
$$

(this implies in particular that $\omega(t, t)=0$ for any $t \in[0, T]$ ).

If $E$ is equipped with a family of semi-norms, and $\alpha>0$, we denote by $\mathcal{V}_{1}^{\alpha}(0, T ; E)$ the set of continuous paths $g:[0, T] \rightarrow E$, such that for each semi-norm $\gamma$, there exist a control $\omega_{\gamma}: \Delta \rightarrow \mathbb{R}_{+}$with

$$
\gamma\left(\delta g_{s t}\right) \leq \omega_{\gamma}(s, t)^{\alpha},
$$

for every $(s, t) \in \Delta$. Similarly, we denote by $\mathcal{V}_{2}^{\alpha}(0, T ; E)$ the set of 2-index maps $g: \Delta \rightarrow$ $E$ such that $g_{t t}=0$ for every $t \in[0, T]$ and

$$
\gamma\left(g_{s t}\right) \leq \omega_{\gamma}(s, t)^{\alpha},
$$

for all $(s, t) \in \Delta$, and some family of controls $\omega_{\gamma}$. If $E$ is a Banach space and $\gamma=|\cdot|_{E}$, one defines a norm $\llbracket \cdot \rrbracket \mathcal{V}_{2}^{\alpha}$ on $\mathcal{V}_{2}^{\alpha}(0, T ; E)$ by taking the infimum of $\omega(0, T)^{\alpha}$ over every possible control $\omega$ such that (2.4) holds. This quantity is in fact equal to the usual $q$-variation norm where $q:=\frac{1}{\alpha}$, as seen for instance in [38, Lemma 3.2].

By $\mathcal{V}_{2, \mathrm{loc}}^{\alpha}(0, T ; E)$ we denote the space of maps $g: \Delta \rightarrow E$ such that there exists a countable covering $\left\{I_{k}\right\}_{k}$ of $I$ satisfying $g \in \mathcal{V}_{2}^{\alpha}\left(I_{k} ; E\right)$ for any $k$. We also define the set $\mathcal{V}_{2}^{1+}(0, T ; E)$ of "negligible remainders" as

$$
\mathcal{V}_{2}^{1+}(0, T ; E):=\bigcup_{\alpha>1} \mathcal{V}_{2}^{\alpha}(0, T ; E),
$$

and similarly for $\mathcal{V}_{2, \text { loc }}^{1+}(0, T ; E)$.

\subsection{Rough drivers}

Before giving definitions, let us quickly explain our approach. For simplicity, let $A=0$, assume that $f$ is smooth and consider a family $B_{t}:=\left(X_{t}(x) \cdot \nabla+X_{t}^{0}(x)\right)$ of first-order differential operators, where for each $i=0 \ldots d, X_{t}^{i}(x)$ is smooth with respect to $x$ (for fixed $t$ ), and $\alpha$-Hölder in $t$ for each $x$, while $\alpha>1 / 3$. Integrating formally (1.3) in time, we have

$$
\begin{aligned}
u_{t}-u_{s}-\int_{s}^{t} f_{r} \mathrm{~d} r= & \left(B_{t}-B_{s}\right) u_{s}+\int_{s}^{t} \mathrm{~d} B_{r}\left(u_{r}-u_{s}\right) \\
= & \delta B_{s t} u_{s}+\iint_{s<r_{1}<r<t} \mathrm{~d} B_{r}\left[\mathrm{~d} B_{r_{1}} u_{r_{1}}+\mathrm{d} r_{1} f_{r_{1}}\right] . \\
= & B_{s t}^{1} u_{s}+\left(\iint_{s<r_{1}<r<t} \mathrm{~d} B_{r} \circ \mathrm{d} B_{r_{1}}\right) u_{s} \\
& +\left(\iint_{s<r_{1}<r<t} \mathrm{~d} B_{r} \circ \mathrm{d} B_{r_{1}}\right)\left[u_{r_{1}}-u_{s}\right]+o(t-s) .
\end{aligned}
$$


One expects any "reasonable" solution to satisfy an estimate of the form $\left|u_{t}-u_{S}\right|_{W^{-1, p}} \lesssim$ $(t-s)^{\alpha}$, so that in particular

$$
\left|\left(\iint_{s<r_{1}<r<t} \mathrm{~d} B_{r} \circ \mathrm{d} B_{r_{1}}\right)\left(u_{r_{1}}-u_{s}\right)\right|_{W^{-3, p}} \lesssim(t-s)^{3 \alpha}=o(t-s) .
$$

Combined with the above, we thus find the Euler-Taylor type expansion

$$
u_{s t}^{\natural}:=\delta u_{s t}-\int_{s}^{t} f_{r} \mathrm{~d} r-\left(B_{s t}^{1}+B_{s t}^{2}\right) u_{s} \in o(t-s),
$$

where we introduce the two-index map $\mathbf{B}=\left(B^{1}, B^{2}\right)$ defined as

$$
\left\{\begin{array}{l}
B_{s t}^{1}:=B_{t}-B_{s}, \\
B_{s t}^{2}:=\iint_{s<r_{1}<r<t} \mathrm{~d} B_{r} \circ \mathrm{d} B_{r_{1}}, \quad 0 \leq s \leq t \leq T .
\end{array}\right.
$$

When $\alpha>1 / 2$, the operators $B_{s t}^{2}$ are canonically defined via an immediate noncommutative generalization of Young Theorem [59]. This is in contrast with the case $\alpha \leq 1 / 2$, where (2.6) does not make sense in general. Indeed, while the definition of $B_{s t}^{1}$ seems not problematic for $B$ continuous (just let $B_{s t}^{1}:=\delta B_{s t}$ ), this is not the case of the second component in general. If $B(n) \rightarrow B$ uniformly on $[0, T]$, a limit point of $\left\{\iint_{s<r_{1}<r_{2}<t} \mathrm{~d} B_{r_{2}}(n) \circ \mathrm{d} B_{r_{1}}(n), n \in \mathbb{N}\right\}$, if it exists, will depend on the choice of the approximating sequence. On the other hand, any limit ought to satisfy the constraint

$$
B_{s t}^{2}-B_{s \theta}^{2}-B_{\theta t}^{2}=B_{\theta t}^{1} \circ B_{s \theta}^{1}, \quad \text { for any } 0 \leq s \leq \theta \leq t \leq T,
$$

which reflects the linearity of the integral, and its additivity with respect to the domain of integration. An essential insight of rough paths theory is that, assuming that $B_{s t}^{2}$ is given with (2.14) together with suitable analytic conditions, then one can simply define the solution $u$ to (1.3) by the Euler-Taylor expansion (2.5). Following Davie's interpretation of rough differential equations [14], we will therefore say that $u$ is a solution to (2.19) if (2.5) holds. The fact that such expansion is sufficient to fully caracterize the solution $u$ is not obvious, and is in fact a consequence of the so-called "Sewing Lemma", which for convenience will be stated in Proposition 3.1.

The previous discussion depicts a non-commutative generalization of the usual rough paths theory, which has been already discussed e.g. in $[4,5,13,21]$. In this picture, real numbers - in which the coordinates of a path $Z:[0, T] \rightarrow \mathbb{R}^{m}$ live - are substituted by elements of an algebra (here a space of differential operators), and the constraint (2.7) corresponds to Chen's relations. What plays here the role of the driving rough path for controlled differential equations is the pair $\mathbf{B}=\left(B^{1}, B^{2}\right)$. It is called an unbounded rough driver (URD), and was first considered by Bailleul and Gubinelli [4] (see also [19, 38, 39]). In the present work, we chose to restrict our attention to a subclass of URDs that are given by differential operators. Such objects will be referred to as differential URDs (or simply "differential rough drivers"). In the sequel we will denote by $\mathbb{D}_{i}, i=1,2$, the space of differential operators of order $i$, that is:

$$
\left[\begin{array}{r}
\mathbb{D}_{1}:=\left\{X^{i}(x) \partial_{i}+Y(x), \text { such that }(X, Y) \in W^{3, \infty} \times W^{2, \infty}\right\}, \\
\mathbb{D}_{2}:=\left\{\mathbb{X}^{i j}(x) \partial_{i j}+\mathbb{Y}^{i}(x) \partial_{i}+\mathbb{Z}(x),\right. \\
\text { such that } \left.(\mathbb{X}, \mathbb{Y}, \mathbb{Z}) \in W^{3, \infty} \times W^{2, \infty} \times W^{1, \infty}\right\} .
\end{array}\right.
$$


The space-regularity of the above coefficients is precisely enough to make sense of (1.3) and obtain energy estimates for it. It is indeed easily seen that the composition of two elements of $\mathbb{D}_{1}$ is an element of $\mathbb{D}_{2}$, while we also have the property that

$$
\mathbb{D}_{i} \subset \bigcap_{k=-3+i}^{3} \mathscr{L}\left(W^{k, p}, W^{k-i, p}\right) \quad \text { for } i=1,2 \text { and } p \in[1, \infty] .
$$

These properties which will be extensively used in the sequel.

We have the following definition.

Definition 2.1 (unbounded rough driver) Let $\alpha>1 / 3$.

A 2-index family $\boldsymbol{B}_{s t} \equiv\left(B_{s t}^{1}, B_{s t}^{2}\right)_{(s, t) \in \Delta}$ of linear operators in $L^{2}\left(\mathbb{R}^{d}\right)$ is called a $\mathcal{V}^{\alpha}$ unbounded rough driver if and only if:

(URD1) $\quad B^{i}$ takes values in $\cap_{k=-3+i}^{3} \mathscr{L}\left(H^{k}, H^{k-i}\right)$ for $i=1,2$, and there exists a control $\omega_{B}: \Delta \rightarrow \mathbb{R}_{+}$such that

$$
\left|B_{s t}^{i}\right|_{\mathscr{L}\left(H^{k}, H^{k-i}\right)} \leq \omega_{B}(s, t)^{i \alpha},
$$

for every $(s, t) \in \Delta$, any $i \in\{1,2\}$ and $k=-3+i, \ldots, 3$.

(URD2) Chen's relations hold true, namely, for every $(s, \theta, t) \in \Delta_{2}$, we have in the sense of linear operators:

$$
\delta B_{s \theta t}^{1}=0, \quad \delta B_{s \theta t}^{2}=B_{\theta t}^{1} \circ B_{s \theta}^{1} .
$$

Moreover, we will say that

(URD $\star) \quad \boldsymbol{B}$ is differential if $\boldsymbol{B}$ is an unbounded rough driver such that

$$
B_{s t}^{i} \in \mathbb{D}_{i}, \quad \text { for } i=1,2 \text { and }(s, t) \in \Delta .
$$

Finally, let $\left(E_{k}\right)_{k \in I}$ be a scale such that there exists $p \in[1, \infty]$ with the property that $E_{k} \hookrightarrow W^{k, p}$ for each $k \in I$. We will say that

(URD $\star \star)$ B acts on the scale $\left(E_{k}\right)_{-3 \leq k \leq 3}$ if

$$
B_{s t}^{i} E_{k} \subset E_{k-i}, \quad-3 \leq k-i \leq k \leq 3, \quad(s, t) \in \Delta,
$$

and if the estimate (2.9) is satisfied with $\left(H^{k}\right)$ being replaced by $\left(E_{k}\right)$.

Remark 2.1 Regarding the definition of $\mathbb{D}_{i}$ for $i=1,2$, any differential, unbounded rough driver can in fact be extended to a family of differential operators acting on the Sobolev scale $\left(W^{k, p}\right)_{-3 \leq k \leq 3}$, for each $p \in[1, \infty]$. For simplicity, in the following we will use the same symbol $\mathbf{B}$ for every such extension.

Note that, if $B:[0, T] \rightarrow \mathbb{D}_{1}$ is a continuous path with finite variation (with respect to the operator-norm of $\cap_{k=-2}^{3} \mathscr{L}\left(H^{k}, H^{k-1}\right)$ ), one can always define the canonical lift $S_{2}(B)$ as the differential rough driver $\mathbf{B} \equiv\left(B^{1}, B^{2}\right)$ given by

$$
S_{2}(B):=\mathbf{B} \text { with }\left\{\begin{array}{l}
B_{s t}^{1}:=B_{t}-B_{s} \in \mathbb{D}_{1} \text { and } \\
B_{s t}^{2}:=\int_{s}^{t} \mathrm{~d} B_{r} \circ\left(B_{r}-B_{s}\right) \in \mathbb{D}_{2} .
\end{array}\right.
$$

The above integral is well-defined in the sense of Riemann-Stieltjes, in the space $\mathbb{D}_{2}$ endowed with the natural operator-norm topology.

This basic observation leads us to the following definition. 
Definition 2.2 (Geometric differential rough driver) Let $p \in[1, \infty]$. Given a differential rough driver $\boldsymbol{B}$ with regularity $\alpha>1 / 3$, we will say that $\boldsymbol{B}$ is geometric if there exists a sequence of paths $B(n) \in C^{1}\left(0, T ; \mathbb{D}_{1}\right), n \geq 0$, such that letting

$$
\boldsymbol{B}(n):=S_{2}(B(n)),
$$

it holds

$$
\begin{aligned}
\rho_{\alpha}(\boldsymbol{B}(n), \boldsymbol{B}):= & \sum_{k=-2}^{3}\|B(n)-B\|_{L^{\infty}\left(0, T ; \mathscr{L}\left(H^{k}, H^{k-1}\right)\right)} \\
& +\sum_{i=1}^{2} \sum_{k=-3+i}^{3} \llbracket B^{i}(n)-B^{i} \rrbracket_{\mathcal{V}^{i \alpha}\left(0, T ; \mathscr{L}\left(H^{k}, H^{k-i}\right)\right) \underset{n \rightarrow \infty}{\longrightarrow} 0 .} .
\end{aligned}
$$

Example 2.1 Recall that a continuous, $m$-dimensional, $q$-rough path with $q=\frac{1}{\alpha}$, is a pair

$$
\mathbf{Z} \equiv\left(Z_{s t}^{1, \mu}, Z_{s t}^{2, \mu \nu}\right)_{\substack{1 \leq \mu, \nu \leq m \\(s, t) \in \Delta}} \text { in } \mathcal{V}_{2}^{\alpha}\left(0, T ; \mathbb{R}^{m}\right) \times \mathcal{V}_{2}^{2 \alpha}\left(0, T ; \mathbb{R}^{m \times m}\right)
$$

such that Chen's relations hold, namely:

$$
\delta Z_{s \theta t}^{1, \mu}=0, \quad \delta Z_{s \theta t}^{2, \mu v}=Z_{s \theta}^{1, \mu} Z_{\theta t}^{1, v}, \quad \text { for }(s, \theta, t) \in \Delta_{2}, \quad 1 \leq \mu, v \leq m .
$$

Roughy speaking, the relations (2.14) indicate that $Z_{s t}^{1, \mu}$ has the form $Z_{t}^{\mu}-Z_{s}^{\mu} \equiv \int_{s}^{t} \mathrm{~d} Z_{r}^{\mu}$ for some path $Z:[0, T] \rightarrow \mathbb{R}^{m}$ while $Z_{s t}^{2, \mu \nu}$ should be thought of as a prescribed value for $\iint_{s<r_{2}<r_{1}<t} \mathrm{~d} Z_{r_{1}}^{v} \mathrm{~d} Z_{r_{2}}^{\mu}$. If $Z$ is smooth, we can define a canonical lift $\mathbf{Z}$ via (2.11), replacing the operation $\circ$ by the tensor product. By definition, the set of geometric rough paths corresponds to the closure of such canonical lifts, with respect to the natural $q$-variation metric. We refer the reader to the monographs $[23,27,51]$ for a thorough introduction to geometric rough paths.

Now, consider a rough path $\mathbf{Z}$, and let $\sigma \in W^{3, \infty}\left(\mathbb{R}^{d} ; \mathbb{R}^{m \times d}\right), \rho \in W^{2, \infty}\left(\mathbb{R}^{d}, \mathbb{R}^{m}\right)$, and for $(s, t) \in \Delta, i=1,2$, define $\mathbf{B} \equiv\left(B^{1}, B^{2}\right)$ as:

$$
\left\{\begin{array}{l}
B_{s t}^{1}:=Z_{s t}^{1, \mu}\left(\sigma_{j}^{\mu} \partial_{j}+\rho^{\mu}\right), \\
B_{s t}^{2}:=Z_{s t}^{2, \mu \nu}\left(\sigma_{j}^{\mu} \partial_{j}+\rho^{\mu}\right)\left(\sigma_{i}^{\nu} \partial_{i}+\rho^{v}\right),
\end{array}\right.
$$

for every $(s, t) \in \Delta$. It is straighforward to check that B satisfies (URD1)-(URD2). Hence it is a differential rough driver. Moreover, it is geometric if $\mathbf{Z}$ is geometric.

Given $B \in \mathcal{V}^{\alpha}\left(0, T ; \mathbb{D}_{1}\right)$, by definition of $\mathbb{D}_{1}$ it is always possible to write $B_{t}$ in terms of some family of bounded and measurable coefficients $X_{t}^{i}(x), i=0, \ldots d$ so that

$$
B_{t}:=X_{t}^{i}(x) \partial_{i}+X_{t}^{0}(x) .
$$

In Appendix A.1, we shall see that there is a one-to-one correspondence between coefficients and elements of $\mathbb{D}_{1}$, and that it yields a continuous isomorphism, see (A.1). In particular, we can assume without any loss of generality that $X^{i} \in \mathcal{V}^{\alpha}\left(0, T ; W^{3, \infty}\right), i=$ $1, \ldots, d$, while $X^{0} \in \mathcal{V}^{\alpha}\left(0, T ; W^{2, \infty}\right)$. For notational convenience, in the remainder of the paper we shall assume that $B$ has the form (2.15). Moreover, we will make use of the shorthand notation

$$
X_{s t}:=X_{t}-X_{s}
$$

hence blurring the difference between the value $X_{t}(x)$ of the coefficient path associated with $B_{t}$ and that of its increments $\delta X_{s t}(x)$.

It turns out that, for geometric differential rough drivers, there is an ensemble of very convenient algebraic rules, as illustrated in the following result. We insist on the fact that 
these rules are a consequence of the geometricity assumption: no further assumption is required on $\mathbf{B}$. The proof of the following lemma is rather simple and merely algebraic, hence we postpone it until Appendix A.1.

Lemma 2.1 Let $\boldsymbol{B}$ be a geometric differential rough driver such that $B_{s t}^{1}=\delta B_{s t}$ where $B_{t}$ is as before. The following assertions are true:

(1) (Weak geometricity I) There exist coefficients $\mathbb{L}^{i} \in \mathcal{V}_{2}^{2 \alpha}\left(W^{2, \infty}\right), i=0, \ldots d$ such that

$$
B_{s t}^{2}=\frac{1}{2} X_{s t}^{i} X_{s t}^{j} \partial_{i j}+\left(\mathbb{L}_{s t}^{i}+X_{s t}^{0} X_{s t}^{i}\right) \partial_{i}+\mathbb{L}_{s t}^{0}+\frac{1}{2}\left(X_{s t}^{0}\right)^{2}
$$

(2) (Generalized Chen's relations) For each $(s, \theta, t) \in \Delta_{2}$, a.e. in $\mathbb{R}^{d}$, it holds

$$
\delta \mathbb{L}_{s \theta t}^{i}=X_{\theta t}^{j} \partial_{j}\left(X_{s \theta}^{i}\right), \quad i=0, \ldots d .
$$

(3) (Weak geometricity II) We have

$$
B_{s t}^{2}=\frac{1}{2} B_{s t}^{1} \circ B_{s t}^{1}+[\boldsymbol{B}]_{s t}
$$

where the "bracket" $[\boldsymbol{B}]$ is a family of first-order differential operators, explicitly given by:

$$
[\boldsymbol{B}]_{s t}:=\left(\mathbb{L}_{s t}^{i}-\frac{1}{2} X_{s t}^{j} \partial_{j} X_{s t}^{i}\right) \partial_{i}+\mathbb{L}_{s t}^{0}-\frac{1}{2} X_{s t}^{j} \partial_{j} X_{s t}^{0}
$$

Notation 2.1 For convenience, we will summarize the above properties by using the shorthand notation

$$
\boldsymbol{B} \sim \boldsymbol{X}=\left(X^{i}, \mathbb{L}^{i}\right)_{i=0, \ldots, d} .
$$

Remark 2.2 If ( $t \mapsto X_{t} \in W^{3, \infty}$ ) has finite variation, $\mathbb{L}$ is explicitly given as

$$
\mathbb{L}_{s t}^{i}:=\int_{s}^{t} \mathrm{~d} X_{r} \cdot \nabla\left(X_{s r}^{i}\right), \quad i=0, \ldots d .
$$

Roughly speaking, $\mathbb{L}$ can be thought of as a differential rough driver analogue of the usual Lévy area for rough paths, in the sense that the knowledge of $\mathbb{L}$ is enough to compute the second level $B^{2}$ of $\mathbf{B}$, as is the case for a geometric rough path (see [27, Definition 13.2]).

In fact, if $\mathbf{B}$ is the pair defined in Example 2.1 with $\mathbf{Z}$ geometric and $\rho=0$, a routine calculation shows the identity

$$
\mathbb{L}_{s t} \cdot \nabla=\frac{1}{2} Z_{s t}^{\mu} Z_{s t}^{v}\left(\sigma^{\mu} \cdot \nabla \sigma^{\nu}\right) \cdot \nabla+\frac{1}{2} \mathbb{A}_{s t}^{\mu v}\left[\sigma^{\mu} \cdot \nabla, \sigma^{\nu} \cdot \nabla\right]
$$

where we denote by $\sigma^{\mu} \cdot \nabla:=\sigma^{\mu i} \partial_{i}$, while $\mathbb{A}_{s t}$ is the Lévy area of $\mathbf{Z}$, and $[\cdot, \cdot]$ denotes the usual Lie bracket of vector fields.

Remark 2.3 As for the usual geometric rough paths, the question may arise whether the algebraic constraints (2.1) and (2.1) imply the geometricity of $\mathbf{B}$ (see [27, Chapter 9]). We conjecture that, upon taking $\alpha$ slighlty smaller, and under "reasonable" conditions on the regularity of the coefficients, the answer should be positive. However, we prefer to leave this issue for future investigations. 


\subsection{Notions of solution}

In the whole paper, we consider an ansatz of the form

$$
\begin{array}{r}
\mathrm{d} v=\left(\partial_{i} f^{i}+f^{0}\right) \mathrm{d} t+\mathrm{d} \mathbf{B} g, \quad \text { on }[0, T] \times \mathbb{R}^{d}, \\
v_{0}=v^{0} \in L^{p},
\end{array}
$$

with $\mathbf{B} \sim\left(X^{i}, \mathbb{L}^{i}\right)_{i=0, \ldots, d}$ being geometric. The drift term $f^{i}, i=0, \ldots d$ is $p$-integrable as a mapping from $[0, T]$ into $L^{p}$ for some $p \in[1, \infty)$, and the derivation $\partial_{i}=\frac{\partial}{\partial x_{i}}$ is understood in distribution sense. By assumption, $g$ will be controlled by $B_{t}$, and so the solution $v$ should be. This means the following.

Definition 2.3 Given $g \in L^{\infty}\left(0, T ; L^{p}\right) \cap \mathcal{V}^{\alpha}\left(0, T ; W^{-1, p}\right)$ we will say that $g$ is controlled by $B$, if there exists $g^{\prime} \in L^{\infty}\left(0, T ; L^{p}\right) \cap \mathcal{V}^{\alpha}\left(0, T ; W^{-1, p}\right)$ such that the element $R^{g}$ of $\mathcal{V}_{2}^{\alpha}\left(0, T ; W^{-1, p}\right)$ defined as

$$
R_{s t}^{g}:=\delta g_{s t}-B_{s t}^{1} g_{s}^{\prime}, \quad \text { for every }(s, t) \in \Delta,
$$

verifies

$$
\left\|R^{g}\right\|_{\mathcal{V}_{2}^{2 \alpha}\left(0, T ; W^{-2, p}\right)}<\infty
$$

(notice the loss of a space-derivative in the above). Abusively, we call $g^{\prime}$ "the Gubinelli derivative" of $g$, though $g^{\prime}$ could be non-unique in principle (at least without any further assumption on B, see Section B.1).

That the unknown $v$ should be controlled by $B$ implies in particular boundedness for the path $v:[0, T] \rightarrow L^{p}$ and also weak-star continuity (hence allowing to give a meaning to the initial condition). In a large part of the sequel we will encounter the situation where $v=g=g^{\prime}$ but this fact is not needed in the definition of a solution, so we will keep things on the more general form (2.19) for the moment.

The following notion of solution was introduced in [4], see also [19].

Definition 2.4 (weak-solution) Let $T>0, \alpha \in(1 / 3,1 / 2]$ and fix $p \in[1, \infty]$. Assume that we are given $f^{i} \in L^{1}\left(0, T ; L^{p}\right), i=0, \ldots d$, and that $g$ is controlled by $B$ with Gubinelli derivative $g^{\prime}$, with $g, g^{\prime}$ both belonging to $L^{\infty}\left(0, T ; L^{p}\right)$. A mapping $v:[0, T] \rightarrow L^{p}$ is called an $L^{p}$-weak solution to the rough PDE (2.19) if it fulfills the following conditions

(1) $\quad v:[0, T] \rightarrow L^{p}$ is bounded as a path taking values in $L^{p}$; moreover, $v$ belongs to $\mathcal{V}^{\alpha}\left(0, T ; W^{-1, p}\right)$;

(2) for every $\phi \in L^{p^{\prime}}$ with $1 / p+1 / p^{\prime}=1, \lim _{t \rightarrow 0} \int_{\mathbb{R}^{d}}\left(v_{t}-v^{0}\right) \phi \mathrm{d} x=0$;

(3) for every $\phi \in W^{2, p^{\prime}}$ with $1 / p+1 / p^{\prime}=1$, and every $(s, t) \in \Delta$ :

$$
\begin{aligned}
\int_{\mathbb{R}^{d}} \delta v_{s t} \phi \mathrm{d} x= & \iint_{[s, t] \times \mathbb{R}^{d}}\left(-f^{i} \partial_{i} \phi+f^{0} \phi\right) \mathrm{d} x \mathrm{~d} r \\
& +\int_{\mathbb{R}^{d}}\left(g_{s} B_{s t}^{1, *} \phi+g_{s}^{\prime} B_{s t}^{2, *} \phi\right) \mathrm{d} x+\left\langle v_{s t}^{\natural}, \phi\right\rangle,
\end{aligned}
$$

for some $v^{\natural} \in \mathcal{V}_{2, \mathrm{loc}}^{1+}\left(0, T ; W^{-3, p}\right)$.

The notion of weak solution fulfills the minimal requirements under which remainder estimates (and thus estimates on rough integrals) can be obtained, see Proposition 3.3. In the sequel however, we will mostly work in a parabolic context, where solutions happen to 
live in a "better space" than the one described above. This motivates the introduction of the following.

Definition 2.5 (Energy solution) Letting $p, p^{\prime} \in[1, \infty]$ so that $1 / p+1 / p^{\prime}=1$, we will say that $v$ is an $L^{p}$-energy solution of (2.19) if it is a weak solution such that additionally

$$
v \in L^{p}\left(0, T ; W^{1, p}\right) .
$$

Similarly, we will say that $v$ is a $L_{\mathrm{loc}}^{p}$-energy solution (or $L^{p}(U)$-energy solution if $U \subset$ $\mathbb{R}^{d}$ ) if it fulfills the above properties, where each occurence of the Sobolev spaces in the space-like variable is replaced by its local counterpart.

\subsection{Rough parabolic equations}

In this section, we consider the rough parabolic equation

$$
\left\{\begin{array}{l}
\mathrm{d} u_{t}-\left(A_{t} u+f_{t}(x)\right) \mathrm{d} t=\left(\mathrm{d} \mathbf{X}_{t}^{i} \partial_{i}+\mathrm{d} \mathbf{X}_{t}^{0}\right) u_{t}, \quad \text { on }(0, T] \times \mathbb{R}^{d}, \\
u_{0}=u^{0} \in L^{p}\left(\mathbb{R}^{d}\right),
\end{array}\right.
$$

where

$$
A_{t}=\partial_{i}\left(a^{i j}(t, \cdot) \partial_{j} \cdot\right)
$$

is given, and we assume the following on $a$.

Assumption 2.1 The coefficients $a=\left(a^{i j}\right)_{1 \leq i, j \leq d}$ are measurable, symmetric in $i$ and $j$ and moreover there exists a constant $\lambda>0$ such that for a.e. $(t, x) \in[0, T] \times \mathbb{R}^{d}$ :

$$
\lambda \sum_{i=1}^{d} \xi_{i}^{2} \leq \sum_{1 \leq i, j \leq d} a^{i j}(t, x) \xi_{i} \xi_{j} \leq \lambda^{-1} \sum_{i=1}^{d} \xi_{i}^{2}, \text { for all } \xi \in \mathbb{R}^{d} .
$$

Concerning the rough part, the following hypotheses will be assumed throughout the paper.

Assumption 2.2 For some fixed $\alpha>1 / 3$, we are given a coefficient path

$$
\left(t \mapsto\left(X_{t}^{i}\right)_{i=0, \ldots, d}\right) \in \mathcal{V}^{\alpha}\left(0, T ; W^{2, \infty} \times\left(W^{3, \infty}\right)^{d}\right),
$$

while $\left(t \mapsto X^{0}\right) \in \mathcal{V}^{\alpha}\left(0, T ; W^{2, \infty}\right)$. These coefficients are given together with a two parameter family

$$
\left.\left((s, t) \mapsto\left(\mathbb{L}_{s t}^{i}\right)_{i=0, \ldots, d}\right)\right) \in \mathcal{V}_{2}^{2 \alpha}\left(0, T ; W^{1, \infty} \times\left(W^{2, \infty}\right)^{d}\right)
$$

which satisfies the generalized Chen's relation

$$
\delta \mathbb{L}_{s \theta t}^{i}=X_{\theta t}^{j} \partial_{j}\left(X_{s \theta}^{i}\right)
$$

for each $0 \leq s \leq \theta \leq t \leq T$ and $i=0, \ldots, d$.

We then let $B_{t}:=X_{t} \cdot \nabla+X_{t}^{0}$ and define a differential rough driver $\boldsymbol{B} \sim \boldsymbol{X}=(X, \mathbb{L})$ as in Lemma 2.1. Hence, it corresponds to the pair $\left(B^{1}, B^{2}\right)$ where

$$
\left[\begin{array}{l}
B_{s t}^{1}=B_{t}-B_{s}=X_{s t}^{i} \partial_{i}+X_{s t}^{0}, \\
B_{s t}^{2}=\frac{1}{2} X_{s t}^{i} X_{s t}^{j} \partial_{i j}+\left(\mathbb{L}_{s t}^{i}+X_{s t}^{0} X_{s t}^{i}\right) \partial_{i}+\mathbb{L}_{s t}^{0}+\frac{1}{2}\left(X_{s t}^{0}\right)^{2} \quad(s, t) \in \Delta,
\end{array}\right.
$$

where we recall that $X_{s t}:=X_{t}-X_{s}$.

Furthermore, we assume that $\boldsymbol{B}$ is geometric, in the sense of Definition 2.2. 
Our first result is about the solvability of (2.24), and completes the results obtained in the previous work [38]. The proof will be given in Section 5 .

Theorem 2.1 Let $f \in L^{2}\left(0, T ; H^{-1}\right)$, fix $u_{0} \in L^{2}$ and consider a geometric, differential rough driver $\boldsymbol{B} \sim\left(X^{i}, \mathbb{L}^{i}\right)_{i=0, \ldots, d}$ as in Assumption 2.2. There exists a unique $L^{2}$-energy solution $u=u\left(u_{0}, f ; \boldsymbol{B}\right)$ to $(2.24)$.

In addition, the solution map is continuous in the following sense

(1) for every $\left(u_{0}, f\right) \in L^{2} \times L^{2}\left(H^{-1}\right)$, the map $\boldsymbol{B} \mapsto u\left(u_{0}, f ; \boldsymbol{B}\right) \in L^{\infty}\left(L^{2}\right) \cap L^{2}\left(H^{1}\right)$ is weakly-star continuous with respect to the rough driver distance $\rho_{\alpha}$ introduced in (2.12).

(2) for B fixed the map $u(\cdot, \cdot ; \boldsymbol{B}): L^{2} \times L^{2}\left(H^{-1}\right) \rightarrow L^{\infty}\left(L^{2}\right) \cap L^{2}\left(H^{1}\right)$ is continuous, with respect to the strong topologies.

Before we state our second main result, we shall first define a set of admissible functions $F: \mathbb{R} \rightarrow \mathbb{R}$ for which right-composition with a solution is possible. We let

$$
C_{\mathrm{adm}}^{2}:=\left\{F \in C^{2}(\mathbb{R} ; \mathbb{R}) \text {, s.t. } F(0)=F^{\prime}(0)=0 \text { and }\left|F^{\prime \prime}\right|_{L^{\infty}}<\infty\right\} .
$$

With this definition, we have the following result.

Theorem 2.2 (Itô Formula) Let A satisfying Assumption 2.1, let $\boldsymbol{B} \sim(X, \mathbb{L})$ such that Assumption 2.2 holds with $X^{0}=0$. Let $u$ be an $L^{2}$-energy solution of (2.24). The following assertions are true.

(i) For every $F \in C_{\text {adm }}^{2}$ it holds the chain rule

$$
\mathrm{d} F(u)=F^{\prime}(u)(A u+f) \mathrm{d} t+\mathrm{d} \boldsymbol{B}[F(u)],
$$

in the sense that the path $[0, T] \rightarrow L^{1}, t \mapsto F\left(u_{t}\right)$ is controlled by $B$ with Gubinelli derivative $\left(F\left(u_{t}\right)\right)^{\prime}=F\left(u_{t}\right)$ and is an $L^{1}$-energy solution to the above equation. More explicitly, we have for any $\phi \in W^{3, \infty}$ and $0 \leq s \leq t \leq T$ :

$$
\begin{array}{r}
\int_{\mathbb{R}^{d}} \delta F(u)_{s t} \phi \mathrm{d} x+\iint_{[s, t] \times \mathbb{R}^{d}}\left[F^{\prime \prime}(u) a^{i j} \partial_{i} u \partial_{j} u \phi+F^{\prime}(u) a^{i j} \partial_{i} u \partial_{j} \phi\right] \mathrm{d} x \mathrm{~d} r \\
=\int_{\mathbb{R}^{d}} F\left(u_{s}\right)\left(B_{s t}^{2, *}+B_{s t}^{2, *}\right) \phi \mathrm{d} x+\left\langle F_{s t}^{\natural}, \phi\right\rangle
\end{array}
$$

for a uniquely determined remainder term $F^{\natural} \in \mathcal{V}_{2,1 \mathrm{loc}}^{1+}\left(0, T ; W^{-3,1}\right)$.

(ii) If $F \in C^{2}$, then (2.28) holds locally. Namely, $t \mapsto F\left(u_{t}\right)$ is controlled by $B$ in the $L_{\text {loc }}^{1}$-sense while (2.29) is true for any $\phi \in W_{\mathrm{loc}}^{2, \infty}$ and a remainder $F^{\natural}$ in $\mathcal{V}_{2, \mathrm{loc}}^{1+}\left(0, T ; W_{\mathrm{loc}}^{-3,1}\right)$.

Remark 2.4 The formula (2.28) is by no means trivial, no matter how smooth $F: \mathbb{R} \rightarrow \mathbb{R}$ is as a function. In fact, the rough integral

$$
\int_{s}^{t} D F\left(u_{r}\right)\left[\mathrm{d} u_{r}\right]
$$

is not even well-defined a priori for an $L^{2}$-energy solution $u$ of (2.24), and this is so regardless of the regularity of $F$.

To wit, note that the expression (2.30) implicitly assumes that $u:[0, T] \rightarrow L^{2}$ is enhanced to a rough path $\mathfrak{u}=\left(\mathfrak{u}^{1}, \mathfrak{u}^{2}\right)$. In particular, one aims to find a topological vector 
space $K$ such that $L^{2}$ is continuously embedded in $K$ and such that $\mathfrak{u}^{i}:[0, T]^{2} \rightarrow K^{\otimes i}$, for $i=1,2$. Leaving aside the question of the choice of tensor product for $K^{\otimes 2}$ (and whether a sense can be given to the rough integral $\mathfrak{u}_{s t}^{2} \equiv \int_{s}^{t} \delta u_{s r} \otimes \mathrm{d} u_{r}$ in $K^{\otimes 2}$ ), we see that $K$ must be chosen such that

$$
\mathfrak{u}^{1} \equiv \delta u \in \mathcal{V}_{2}^{\alpha}(0, T ; K) .
$$

For an $L^{2}$-energy solution $u$, we only expect that $\delta u \in \mathcal{V}_{2}^{\alpha}\left(0, T ; H^{-1}\right)$ (see Section 3), and hence the condition (2.31) imposes that $H^{-1} \hookrightarrow K$. In particular, this requires that the nonlinear operator

$$
\bar{F}: H^{-1} \rightarrow L^{1}, \quad u \mapsto \bar{F}(u):=F(u(\cdot))
$$

be of class $C^{1}$, which is cleary not the case of any smooth function $F$.

A core argument in the proof of Theorem 2.2 is the fact that for an appropriate subclass of free terms $f$, the solutions of (2.24) are bounded. This is stated in the following result.

\section{Theorem 2.3 Let}

$$
f \in L^{r}\left(0, T ; L^{q}\right),
$$

where the exponents $r \in(1, \infty]$ and $q \in\left(1 \vee \frac{d}{2}, \infty\right)$ are subject to the conditions

$$
\frac{1}{r}+\frac{d}{2 q}<1
$$

Then, the solution $u$ obtained from Theorem 2.1 is locally bounded, away from $t=0$. Precisely, for any $\tau>0$, and any compact set $K \subset \subset \mathbb{R}^{d}$, it holds the estimate

$$
\|u\|_{L^{\infty}([\tau, T] \times K)} \leq C\left(\tau, K,\left|u_{0}\right|_{L^{2}}, \lambda,\|f\|_{L^{r}\left(L^{q}\right)}, \omega_{B}, \alpha, r, q\right),
$$

where the above constant only depends on the indicated quantities.

Note that the chain rule given in Theorem 2.2 does not apply directly for the $L^{p}$-norm case since $F=|\cdot|^{p}$ is not admissible. Fortunately, we can show the following.

Corollary 2.1 Let $p \geq 2, \boldsymbol{B} \sim \boldsymbol{X}=(X, \mathbb{L})$ be as in Theorem 2.2, and take $f \in$ $L^{p}\left(0, T ; W^{-1, p}\right)$. Assume that $u$ is an $L^{p}$-energy solution of (2.24).

Then, $|u|^{p}$ is an $L^{1}$-energy solution of

$$
\mathrm{d}\left(|u|^{p}\right)=p u|u|^{p-2}(A u+f) \mathrm{d} t+\mathrm{d} \boldsymbol{X} \cdot \nabla\left(|u|^{p}\right) .
$$

In general, when $\mathbf{B}$ is geometric and such that $X^{0} \neq 0$, we can write a similar chain rule for the $L^{p}$-norm of $u$, assuming that $p \geq 4$. This is stated in the next result.

Theorem 2.4 Fix $p \geq 4$, and assume that $\boldsymbol{B} \sim(X, \mathbb{L})$ satisfies Assumption 2.2. For every $f \in L^{1}\left(0, T ; W^{-1, \bar{p}}\right) \cap L^{2}\left(0, T ; H^{-1}\right)$ and $u_{0} \in L^{p}$, there exists a unique $L^{2}$-energy solution $u$ to (2.24) such that $\iint_{[0, T] \times \mathbb{R}^{d}}|u|^{p-2}|\nabla u|^{2} \mathrm{~d} x \mathrm{~d} t<\infty$.

Moreover, it holds in the $L^{1}$-sense:

$$
\mathrm{d}\left(|u|^{p}\right)=p u|u|^{p-2}(A u+f) \mathrm{d} t+\mathrm{d} \boldsymbol{B}^{(p)}\left(|u|^{p}\right) .
$$

where $\boldsymbol{B}^{(p)}$ is given by

$$
\left\{\begin{array}{l}
B_{s t}^{(p), 1}:=X_{s t}^{i} \partial_{i}+p X_{s t}^{0} \\
B_{s t}^{(p), 2}:=\frac{1}{2} X_{s t}^{i} X_{s t}^{j} \partial_{i j}+\left(\mathbb{L}_{s t}^{i}+p X_{s t}^{0} X_{s t}^{i}\right) \partial_{i}+p \mathbb{L}_{s t}^{0}+\frac{p^{2}}{2}\left(X_{s t}^{0}\right)^{2} .
\end{array}\right.
$$


Remark 2.5 The previous theorem implies in particular that $L^{p}$-energy solutions are unique, since in that case, Hölder Inequality yields

$$
\left\||u|^{p-2}|\nabla u|^{2}\right\|_{L^{1}\left(0, T ; L^{1}\right)} \leq\left(\sup _{t \in[0, T]}\left|u_{t}\right|_{L^{p}}^{p-2}\right) \int_{0}^{T}\left|\nabla u_{t}\right|_{L^{p}}^{2} d t,
$$

and the above right hand side is finite by assumption. However the existence of $L^{p}$-energy solutions is not guaranteed without any additional assumption.

We now give a by-product of our results concerning the following homogeneous Dirichlet problem with transport noise

$$
\left\{\begin{array}{l}
\mathrm{d} u_{t}-A_{t} u \mathrm{~d} t=\mathrm{d} \mathbf{Z}_{t}^{\mu} \sigma^{\mu}(x) \cdot \nabla u_{t}, \quad \text { on } \mathbb{R}_{+} \times D, \\
u(0)=u_{0}, \\
\left.u_{t}\right|_{\partial D}=0 \quad \text { (trace sense), for all } t \geq 0,
\end{array}\right.
$$

where $\mathbf{Z}^{\mu} \sigma^{\mu}$ is given the enhancement of Example 2.1 with $\rho=0$ and where $\mathbf{Z}$ is geometric. Moreover, we assume that the coefficients $\sigma^{\mu}, \mu=1, \ldots, d$, have compact support in $D$. With this assumption, it is easily seen that $\mathbf{B}$ acts on the scales $\left(W_{0}^{k, p}(D)\right)_{-3 \leq k \leq 3}$ for any $p \in[1, \infty]$, in the sense of Definition 2.1-(URD $\star \star)$.

We have the following result.

Theorem 2.5 (weak maximum principle for (2.35)) Assume that $D \subset \mathbb{R}^{d}$ is an open domain which is smooth and bounded. Let A be as in Assumption 2.1 and define $\mathbf{Z} \sigma \cdot \nabla$ as above. Assume furthermore that

$$
\sigma \in W_{0}^{3, \infty}\left(D ; \mathbb{R}^{m \times d}\right) .
$$

There exists a unique solution $u$ of the Dirichlet problem (2.35), by which we mean that $u$ is an $L^{2}(D)$-energy solution with the following additional property

$$
u \in L^{2}\left(0, T ; W_{0}^{1,2}(D)\right) .
$$

Moreover, $u$ belongs to $\in L^{\infty}([0, T] \times D)$ and we have the following maximum principle for $u$ :

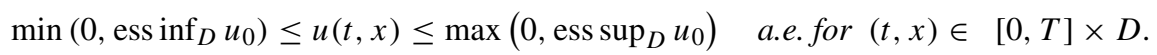

\section{Controlled paths}

\subsection{Some useful facts}

For pedagogical purposes, we first recall some elements of Rough Path Theory from the point of view adopted in [32]. The main problem addressed by this theory is, roughly speaking, to give a meaning to incremental equations of the form

$$
u_{t}-u_{s}=\int_{s}^{t} H, \quad \text { for }(s, t) \in \Delta, \quad\left(u_{0} \text { given }\right),
$$

where $\Delta \ni(s, t) \mapsto H_{s t}$ is a "jet" associated to the quantity one wishes to integrate. A concrete example is given by the Riemmann-Stieljes integral $\int_{s}^{t} H \equiv \int_{s}^{t} f_{r} \mathrm{~d} Z_{r}$ where $f$ and 
$Z$ are $\alpha$-Hölder with $\alpha>1 / 2$, an associated first order approximation of which is provided by the jet

$$
H_{s t}:=f_{s} \delta Z_{s t}
$$

The value of $\int_{s}^{t} f \mathrm{~d} Z$ is obtained by taking the limit of the Riemann sums $\sum_{i=1}^{n} H_{t_{i} t_{i+1}}$ as $n \rightarrow \infty$ and $\max \left|t_{i+1}-t_{i}\right| \rightarrow 0$. Suppose now that the integrand $f$ is itself expressed as an integral against $Z$, say $\delta f_{s t}:=\int_{s}^{t} g \mathrm{~d} Z$ for some $g \in C^{1}$. Then, a better approximation of the former integral is given by the Milstein-type jet

$$
\tilde{H}_{s t}:=f_{s} \delta Z_{s t}+g_{s} \int_{s}^{t} \delta Z_{s r} \mathrm{~d} Z_{r},
$$

as easily seen by Taylor formula. When $\alpha \leq 1 / 2$, the first choice (3.2) may generate divergent Riemann sums, which leads us to investigate generalizations of (3.3). If $Z$ is endowed with an enhancement to a rough path $\mathbf{Z} \equiv\left(Z^{1}, Z^{2}\right)$, and if we replace the iterated integral in (3.3) by its postulated value $Z_{s t}^{2}$, the expression (3.3) is still meaningful.

The so-called Sewing Lemma [32] asserts that if $\alpha>1 / 3$, then there is a unique couple $\left(u, u^{\natural}\right)$ such that $u_{t}-u_{s}=\tilde{H}_{s t}+u_{s t}^{\natural}$ and

$$
\left|u_{s t}^{\natural}\right| \lesssim(t-s)^{3 \alpha}[\delta \tilde{H}]_{3 \alpha},
$$

where $[\delta \tilde{H}]_{3 \alpha}$ is the generalized $3 \alpha$-Hölder seminorm of the 3-parameter quantity

$$
\delta \tilde{H}_{s \theta t} \equiv \tilde{H}_{s t}-\tilde{H}_{s \theta}-\tilde{H}_{\theta t}, \quad(s, \theta, t) \in \Delta_{2} .
$$

The quantity $\int_{s}^{t} H:=H_{s t}+u_{s t}^{\natural}$ is called the rough integral of $H$, and it is consistent with usual Riemann-Stieljes integration when $H_{s t}=f_{s} \delta Z_{s t}$.

The following result, which is of fundamental importance in this paper, summarizes what we discussed above. In the statement below, we assume for simplicity that $E$ is a Banach space, but it could easily be replaced by a Fréchet space (e.g. the Sobolev spaces $W_{\text {loc }}^{k, p}$, or the Schwartz distributions), with $\omega$ being dependent on the semi-norm considered.

Proposition 3.1 (Sewing Lemma) Let $H: \Delta \rightarrow E$ and $C>0$ be such that

$$
\left|\delta H_{s \theta t}\right| \leq C \omega(s, t)^{a}, \quad 0 \leq s \leq \theta \leq t \leq T
$$

for some $a>1$, and some control function $\omega$, and denote by $[\delta H]_{a, \omega}$ the smallest possible constant $C$ in the above bound.

There exists a unique pair $I:[0, T] \rightarrow E$ and $I^{\natural}: \Delta \rightarrow$ E satisfying

$$
\delta I_{s t}=H_{s t}+I_{s t}^{\natural}
$$

where for $0 \leq s \leq t \leq T$,

$$
\left|I_{s t}^{\natural}\right| \leq C_{a}[\delta H]_{a, \omega} \omega(s, t)^{a},
$$

for some constant $C_{a}$ only depending on a. In fact, I is defined via the Riemann type integral approximation

$$
I_{t}=\lim \sum_{i=1}^{n} H_{t_{i}^{n} t_{i+1}^{n}},
$$

the above limit being taken along any sequence of partitions $\left\{t^{n}, n \geq 0\right\}$ of $[0, t]$ whose mesh-size converges to 0 .

Besides rough integration, one of the main tools that we shall use in the sequel is a Gronwall-type argument which is well-adapted to incremental equations of the form (3.1), but in a more general, $q$-variation context. We will extensively make use of the following version of this result, whose proof is due to [19]. 
Lemma 3.1 (Rough Gronwall) Let $G:[0, T] \rightarrow \mathbb{R}_{+}$be a path such that there exist constants $\kappa, L>0$, a control $\omega$, and a superadditive map $\varphi$ with:

$$
\delta G_{s t} \leq\left(\sup _{s \leq r \leq t} G_{r}\right) \omega(s, t)^{\kappa}+\varphi(s, t),
$$

for every $(s, t) \in \Delta$ under the smallness condition $\omega(s, t) \leq L$.

Then, there exists a constant $\tau_{\kappa, L}>0$ such that

$$
\sup _{0 \leq t \leq T} G_{t} \leq \exp \left(\frac{\omega(0, T)}{\tau_{\kappa, L}}\right)\left[G_{0}+\sup _{0 \leq t \leq T}|\varphi(0, t)|\right] .
$$

\subsection{Integration in $\mathcal{D}_{B}^{\alpha, p}$}

In this paragraph we consider a smooth domain $U \subset \mathbb{R}^{d}$ and we fix $p, p^{\prime} \in[1, \infty]$ so that $1 / p+1 / p^{\prime}=1$. For notational simplicity, we will omit the domain of integrability and denote by $L^{p}=L^{p}(U), W^{k, p}=W^{k, p}(U)$, and so on. In the remainder of the section, we will assume that

$$
\mathbf{B} \equiv\left(B^{1}, B^{2}\right) \quad \text { is a } \mathcal{V}^{\alpha} \text {-unbounded rough driver acting on the scale }\left(W^{k, p}\right)_{k=-3}^{3},
$$

under the assumption that $\alpha>1 / 3$.

For $k \geq 0$, and $y \in \mathcal{V}_{2}^{k \alpha}\left(0, T ; W^{-k, p}\right)$, we shall use the notations

$$
\llbracket y \rrbracket_{-k}^{[k \alpha]}(s, t):=\|y\|_{\mathcal{V}_{2}^{k \alpha}\left(s, t ; W^{-k, p}\right)}, \quad \text { for }(s, t) \in \Delta,
$$

and

$$
\llbracket y \rrbracket_{-k}^{[k \alpha]}:=\llbracket y \rrbracket_{-k}^{[k \alpha]}(0, T) .
$$

These are motivated by the (tautological) fact that for $y$ as above the quantity $\omega(s, t):=$ $\llbracket y \rrbracket_{-k}^{[k \alpha]}(s, t)^{\frac{1}{k \alpha}}$ defines a control which is larger than $\left|\delta y_{s t}\right|_{W^{-k, p}}$ for each $(s, t) \in \Delta$ (it is in fact the smallest one).

We now introduce what in the context of unbounded rough drivers plays the role of the usual controlled path space. Note that the definition below only makes use of the first level $B=B_{0}^{1}$. of $\mathbf{B}$, which is why we write $\mathcal{D}_{B}^{\alpha, p}$ instead of $\mathcal{D}_{\mathbf{B}}^{\alpha, p}$.

Definition 3.1 (Controlled path space) Given $\alpha \in(1 / 3,1 / 2]$, we define the controlled path space $\mathcal{D}_{B}^{\alpha, p} \equiv \mathcal{D}_{B}^{\alpha, p}([0, T] \times U)$ as the linear space of couples

$$
\left(g, g^{\prime}\right) \in\left(L^{\infty}\left(0, T ; L^{p}\right) \cap \mathcal{V}^{\alpha}\left(0, T ; W^{-1, p}\right)\right)^{2}
$$

such that $g$ is controlled by $B$ with Gubinelli derivative $g^{\prime}$ (in the sense of Definition 2.3).

Furthermore, equipped with the norm

$$
\left\|\left(g, g^{\prime}\right)\right\|_{\mathcal{D}_{B}^{\alpha, p}([0, T] \times U)}:=\left\|\left(g, g^{\prime}\right)\right\|_{L^{\infty}\left(0, T ; L^{p}(U)\right)}+\llbracket R^{g} \rrbracket_{-2}^{[2 \alpha]}+\llbracket \delta g^{\prime} \rrbracket_{-1}^{[\alpha]},
$$

the space $\mathcal{D}_{B}^{\alpha, p}([0, T] \times U)$ forms a Banach space.

Consider $\left(g, g^{\prime}\right) \in \mathcal{D}_{B}^{\alpha, p}$ and let $f \in L^{1}\left(0, T ; W^{-3, p}\right)$. Applying Proposition 3.1 with the choices

$$
E:=W^{-3, p}, \quad H_{s t}:=\int_{s}^{t} f_{r} \mathrm{~d} r+B_{s t}^{1} g_{s}+B_{s t}^{2} g_{s}^{\prime},
$$


it is easily seen that there exists a unique couple $\left(u, u^{\natural}\right) \in C\left(0, T ; W^{-3, p}\right) \times$ $\mathcal{V}_{2}^{1+}\left(0, T ; W^{-3, p}\right)$ such that for any $(s, t) \in \Delta$ :

$$
u_{t}-u_{s}=\int_{s}^{t} f_{r} \mathrm{~d} r+B_{s t}^{1} g_{s}+B_{s t}^{2} g_{s}^{\prime}+u_{s t}^{\natural} .
$$

Indeed, we have using Chen's relations

$$
-\delta H_{s \theta t}=B_{\theta t}^{1} R_{s \theta}^{g}+B_{\theta t}^{2} \delta g_{s \theta}^{\prime}, \quad(s, \theta, t) \in \Delta_{2},
$$

and therefore

$$
\left|\delta H_{s \theta t}\right|_{W^{-3, p}} \lesssim \omega_{B}(s, t)^{\alpha} \llbracket R^{g} \rrbracket_{-2}^{[2 \alpha]}(s, t)+\omega_{B}(s, t)^{2 \alpha} \llbracket \delta g^{\prime} \rrbracket_{-1}^{[\alpha]}(s, t),
$$

which is finite by definition of the controlled path space. Hence, the sewing lemma (Proposition 3.1) applies, which shows existence and uniqueness of $\left(u, u^{\natural}\right)$ satisfying (3.11), as an equality in $W^{-3, p}$.

In the sequel, the following suggestive notation will be adopted

$$
\mathrm{d} u=f \mathrm{~d} t+\mathrm{d} \mathbf{B}\left(g, g^{\prime}\right) .
$$

or simply

$$
\mathrm{d} u=f \mathrm{~d} t+\mathrm{d} \mathbf{B} g
$$

if $g=g^{\prime}$. We point out that (3.13) does not necessarily mean that $u$ is a weak solution, because Definition 2.4 involves some assumptions on the regularity of $u$. The remainder of this section will address these regularity issues.

\subsection{Remainder estimates}

Conversely, starting from the relation (3.13) for some $g \in L^{\infty}\left(0, T ; L^{p}\right)$, one would like to know under which conditions on $f$ and $g$ does the solution $u$ belong to the controlled paths space $\mathcal{D}_{B}^{\alpha, p}$. A first observation in this direction is the following.

Proposition 3.2 Consider $f \in L^{1}\left(0, T ; W^{-2, p}\right)$ let $(g, g) \in \mathcal{D}_{B}^{\alpha, p}$, and assume that $v$ satisfies

$$
\mathrm{d} v=f \mathrm{~d} t+\mathrm{d} \boldsymbol{B} g,
$$

(see (3.11)). Then, $v$ is controlled by $B$ with Gubinelli derivative $v^{\prime}=g$. Moreover, the following estimate holds on $R_{s t}^{v} \equiv \delta v_{s t}-B_{s t}^{1} g_{s}$ :

$$
\begin{aligned}
\llbracket R^{v} \rrbracket_{-2}^{[2 \alpha]}(s, t) \leq C\left[\int_{s}^{t}\left|f_{r}\right|_{W^{-2, p}} \mathrm{~d} r\right. & \left.+\omega_{B}(s, t)^{2 \alpha}\|v, g\|_{L^{\infty}\left(s, t ; L^{p}\right)}\right] \\
& +\frac{1}{2}\left(\llbracket R^{g} \rrbracket_{-2}^{[2 \alpha]}(s, t)+\omega_{B}(s, t)^{\alpha} \llbracket \delta g \rrbracket_{-1}^{[\alpha]}(s, t)\right) .
\end{aligned}
$$

In particular, if one assumes $v=g$, this yields the bound

$$
\llbracket R^{v} \rrbracket_{-2}^{[2 \alpha]}(s, t) \leq 2 C\left[\int_{s}^{t}\left|f_{r}\right|_{W^{-2, p}} \mathrm{~d} r+\omega_{B}(s, t)^{2 \alpha}\|v\|_{L^{\infty}\left(s, t ; L^{p}\right)}+\omega_{B}(s, t)^{\alpha} \llbracket \delta v \rrbracket_{-1}^{[\alpha]}(s, t)\right] .
$$

Before we proceed to the proof of Proposition 3.2, let us observe the following. There exists a family $\left(J_{\eta}\right)_{\eta \in(0,1)}$ of bounded linear maps $J_{\eta} \in L\left(W^{k, p}, W^{k, p}\right), \eta \in(0,1), k \in \mathbb{Z}$ being arbitrary, such that:

$$
\text { - } J_{\eta} \text { maps } W^{k, p} \text { into } C^{\infty} \text {, for every } \eta \in(0,1) \text {. }
$$


For some constant $C_{J}>0$, for any $\ell \in \mathbb{N}_{0}$ with $|k-\ell| \leq 2$ : if $0 \leq k \leq \ell \leq 3$, then

$$
\left|J_{\eta}\right|_{\mathscr{L}\left(W^{k, p}, W^{\ell, p}\right)} \leq \frac{C_{J}}{\eta^{\ell-k}}, \quad \text { for all } \quad \eta \in(0,1) .
$$

Finally, if $0 \leq \ell \leq k \leq 3$, then

$$
\text { - } \quad \mid \text { id }-\left.J_{\eta}\right|_{\mathscr{L}\left(W^{k, p}, W^{\ell, p}\right)} \leq C_{J} \eta^{k-\ell}, \quad \text { for all } \eta \in(0,1) .
$$

In the case when $U \equiv \mathbb{R}^{d}$ and $p \in[1, \infty]$ it suffices to consider $J_{\eta} f:=\eta^{-d} \rho(\dot{\bar{\eta}}) * f$, where $\rho$ is a radially symmetric, smooth function integrating to one. For the general case, we refer for instance to [38, Appendix A.3])

From now on, we shall refer to $\left(J_{\eta}\right)_{\eta \in(0,1)}$ as a family of smoothing operators.

With this observation at hand, we can now proceed to the proof of the above result.

Proof of Proposition 3.2 Note that

$$
R_{s t}^{v}:=\delta v_{s t}-B_{s t}^{1} g_{s} \equiv \int_{s}^{t} f_{r} \mathrm{~d} r+B_{s t}^{2} g_{s}+v_{s t}^{\natural} .
$$

Using (3.16)-(3.17), we can interpolate these two different expressions for $R^{g}$, by writing

$$
\begin{aligned}
\left|R_{s t}^{v}\right|_{W^{-2, p}} \leq & \left|J_{\eta}\left(\int_{s}^{t} f \mathrm{~d} r+B_{s t}^{2} g_{s}+v_{s t}^{\natural}\right)\right|_{W^{-2, p}}+\left|\left(\mathrm{id}-J_{\eta}\right)\left[\delta v_{s t}-B_{s t}^{1} g_{s}\right]\right|_{W^{-2, p}} \\
\lesssim & \left|\int_{s}^{t} f_{r} \mathrm{~d} r\right|_{W^{-2, p}}+\left|B_{s t}^{2} g\right|_{W^{-2, p}}+\frac{\left|v_{s t}^{\natural}\right|_{W^{-3, p}}}{\eta} \\
& +\eta^{2} 2\|v\|_{L^{\infty}\left(L^{p}\right)}+\eta \omega_{B}(s, t)^{\alpha}\|g\|_{L^{\infty}\left(L^{p}\right) .}
\end{aligned}
$$

In order to estimate $v^{\natural}$, note that Chen's relations (2.10) imply $-\delta\left(B^{1} g+B^{2} g\right)_{s \theta t}=B_{\theta t}^{1}\left(\delta g_{s \theta}-B_{s \theta}^{1} g_{s}\right)+B_{\theta t}^{2} \delta g_{s \theta}=B_{\theta t}^{1} R_{s \theta}^{g}+B_{\theta t}^{2} \delta g_{s \theta}, \quad$ for $(s, \theta, t) \in \Delta_{2}$.

From this and the Sewing Lemma, we infer that

$$
\left|v_{s t}^{\natural}\right|_{W^{-3, p}} \leq C(\alpha)\left(\omega_{B}(s, t)^{\alpha} \llbracket R^{v} \rrbracket_{-2}^{[2 \alpha]}(s, t)+\omega_{B}(s, t)^{2 \alpha} \llbracket \delta v \rrbracket_{-1}^{[\alpha]}(s, t)\right) .
$$

Now, since (3.18) is true for arbitrary $\eta \in(0,1)$, we can choose $\eta:=\zeta \omega_{B}(s, t)^{\alpha}$ for some $\zeta>0$ big enough. We obtain from (3.19):

$$
\begin{aligned}
\left|R_{s t}^{v}\right|_{W^{-2, p}} \leq & \left(\int_{s}^{t}\left|f_{r}\right|_{W^{-2, p}} \mathrm{~d} r\right)+\omega_{B}(s, t)^{2 \alpha}\|v, g\|_{L^{\infty}\left(s, t ; L^{p}\right)}+\frac{\llbracket v^{\natural} \rrbracket_{-3}^{[3 \alpha]}(s, t)}{\zeta \omega_{B}(s, t)^{\alpha}} \\
\leq & \left(\int_{s}^{t}\left|f_{r}\right|_{W^{-2, p}} \mathrm{~d} r\right)+\omega_{B}(s, t)^{2 \alpha}\|v, g\|_{L^{\infty}\left(s, t ; L^{p}\right)} \\
& +\frac{1}{2}\left(\llbracket R^{g} \rrbracket_{-2}^{[2 \alpha]}(s, t)+\omega_{B}(s, t)^{\alpha} \llbracket \delta g \rrbracket_{-1}^{[\alpha]}(s, t)\right),
\end{aligned}
$$

provided that $\omega_{B}(s, t) \leq L(\alpha)$.

This shows the claimed property.

Consider an equation of the form $\mathrm{d} v=f \mathrm{~d} t+\mathrm{d} \mathbf{B} v$, with $f \in L^{1}\left(0, T ; W^{-2, p}\right)$, and define the remainder $u^{\natural} \in \mathcal{V}_{2}^{1+}\left(0, T ; W^{-3, p}\right)$ as in (3.11), namely

$$
v_{s t}^{\natural}:=\delta v_{s t}-\int_{s}^{t} f_{r} \mathrm{~d} r-\left(B_{s t}^{1}+B_{s t}^{2}\right) v_{s}, \quad(s, t) \in \Delta .
$$


As was observed in [19], it is possible in this case to obtain a priori estimates on $v^{\natural}$ in $\mathcal{V}^{3 \alpha}\left(W^{-3, p}\right)$, explicitly in terms of $\|f\|_{L^{1}\left(0, T ; W^{-2, p}\right)}$ and $\|v\|_{L^{\infty}\left(L^{p}\right)}$ only. This is the content of the following result, which will be an essential tool in the sequel.

Proposition 3.3 (Remainder estimates) Fix $\alpha \in(1 / 3,1 / 2], p \in[1, \infty]$ and let $v \in$ $L^{\infty}\left(0, T ; L^{p}\right)$ such that

$$
\mathrm{d} v=f \mathrm{~d} t+\mathrm{d} \boldsymbol{B} v
$$

for some $f \in L^{p}\left(0, T ; W^{-2, p}\right)$.

Then, the remainder $v^{\natural}$ defined by (3.20) has locally finite $\frac{1}{3 \alpha}$-variation. Moreover, there are constants $C, L>0$ depending only on $\alpha$, such that for each $(s, t) \in \Delta$ satisfying

$$
\omega_{B}(s, t) \leq L,
$$

it holds

$$
\llbracket v^{\natural} \rrbracket_{-3}^{[3 \alpha]}(s, t) \leq C\left(\omega_{B}(s, t)^{3 \alpha}\|v\|_{L^{\infty}\left(s, t ; L^{p}\right)}+\omega_{B}(s, t)^{\alpha} \int_{s}^{t}\left|f_{r}\right|_{W^{-2, p}} \mathrm{~d} r\right) .
$$

As a consequence, any $v$ satisfying the Euler-Taylor expansion (3.20) is controlled by $B$ with Gubinelli derivative $v^{\prime}=v$, that is $\|(v, v)\|_{\mathcal{D}_{B}^{\alpha, p}}<\infty$. In addition, it holds the a priori estimates

$$
\begin{aligned}
& \llbracket \delta v_{s t} \rrbracket_{-1}^{[\alpha]}(s, t) \leq C\left[\left(\int_{s}^{t}\left|f_{r}\right|_{W^{-2, p}} \mathrm{~d} r\right)^{\alpha}+\omega_{B}(s, t)^{\alpha}\|v\|_{L^{\infty}\left(s, t ; L^{p}\right)}\right] \\
& \llbracket R_{s t}^{v} \rrbracket_{-2}^{[2 \alpha]}(s, t) \leq C\left[\int_{s}^{t}\left|f_{r}\right|_{W^{-2, p}} \mathrm{~d} r+\omega_{B}(s, t)^{2 \alpha}\|v\|_{L^{\infty}\left(s, t ; L^{p}\right)}\right]
\end{aligned}
$$

for any $(s, t) \in \Delta$ such that $\omega_{B}(s, t)+\int_{S}^{t}\left|f_{r}\right|_{W^{-2, p}} \mathrm{~d} r \leq L$, where $L(\alpha)>0$.

Note that (3.22) is implicitly contained in [19]. Since our notations and settings are different, we provide a full proof.

Proof of Proposition 3.3. Proof of (3.22). By definition of a weak solution, there exists some $z \in(1,3 \alpha]$ such that $v^{\natural}$ has finite $1 / z$-variation, namely:

$$
\omega_{z}(s, t):=\llbracket v^{\natural} \rrbracket_{\mathcal{V}_{2}^{z}\left(s, t ; W^{-3, p}\right)}^{1 / z}<\infty .
$$

Furthermore, we recall the following property (see [38]): for any $(s, t) \in \Delta$,

$$
\omega_{z}(s, t)=\inf \left\{\omega(s, t), \omega: \Delta_{[s, t]} \rightarrow \mathbb{R}_{+} \text {control such that }(\omega)^{z} \geq\left|v^{\natural}\right|_{W^{-3, p}}\right\} .
$$

Applying $\delta$ to both sides of (3.20) and making use of Chen's relations (2.10), we have for $(s, \theta, t) \in \Delta_{2}$,

$$
\delta v_{s \theta t}^{\natural}=B_{\theta t}^{1}\left(\delta v_{s \theta}-B_{s \theta}^{1} v_{s}\right)+B_{\theta t}^{2} \delta v_{s \theta} \equiv B_{\theta t}^{1} R_{s \theta}^{v}+B_{\theta t}^{2} \delta v_{s \theta},
$$

by definition of $R^{v}$ in (2.20). Taking the $W^{-3, p}$-norm and then using (3.14), we obtain

$$
\begin{aligned}
& \left|\delta v_{s \theta t}^{\natural}\right|_{W^{-3, p}} \leq \omega_{B}(s, t)^{\alpha} \llbracket R^{v} \rrbracket_{-2}^{[2 \alpha]}(s, t)+\omega_{B}(s, t)^{2 \alpha} \llbracket \delta v \rrbracket_{-1}^{[\alpha]}(s, t) \\
& \lesssim \omega_{B}(s, t)^{\alpha} \int_{s}^{t}|f|_{W^{-2, p}} \mathrm{~d} r+\omega_{B}(s, t)^{3 \alpha}\|v\|_{L^{\infty}\left(s, t ; L^{p}\right)}+\omega_{B}(s, t)^{2 \alpha} \llbracket \delta v \rrbracket_{-1}^{[\alpha]}(s, t)
\end{aligned}
$$


so that the problem boils down to estimating the term $\llbracket \delta v \rrbracket_{-1}^{[\alpha]}(s, t)$. To obtain such an estimate, we proceed as in the proof of Proposition 3.3, writing

$$
\begin{aligned}
\delta v_{s t} & =\left(\mathrm{id}-J_{\eta}\right) \delta v_{s t}+J_{\eta} \delta v_{s t} \\
& =\left(\mathrm{id}-J_{\eta}\right) \delta v_{s t}+J_{\eta}\left(\int_{s}^{t} f_{r} \mathrm{~d} r+B_{s t}^{1} v_{s}+B_{s t}^{2} v_{s}+v_{s t}^{\natural}\right)
\end{aligned}
$$

where $J_{\eta}, \eta \in(0,1)$, denotes a family of smoothing operators. Making use of the properties (3.15)-(3.17) we obtain

$$
\begin{aligned}
\left|\delta v_{s t}\right|_{W^{-1, p}} \lesssim \eta\|v\|_{L^{\infty}\left(s, t ; L^{p}\right)}+ & \frac{1}{\eta} \int_{s}^{t}\left|f_{r}\right|_{W^{-2, p}} \mathrm{~d} r+\omega_{B}(s, t)^{\alpha}\left\|v_{s}\right\|_{L^{\infty} ; L^{p}} \\
& +\frac{\omega_{B}(s, t)^{2 \alpha}}{\eta}\left\|v_{s}\right\|_{L^{\infty}\left(s, t ; L^{p}\right)}+\frac{\omega_{z}(s, t)^{z}}{\eta^{2}}
\end{aligned}
$$

by definition of the control $\omega_{z}$. Going back to (3.26) and making the choice

$$
\eta:=\zeta \omega_{B}(s, t)^{\alpha}
$$

for some parameter $\zeta>0$ (to be fixed later), we obtain the inequality

$$
\begin{gathered}
\left|\delta v_{s \theta t}^{\natural}\right|_{W^{-3, p}} \leq C_{J}\left(\omega_{B}(s, t)^{\alpha} \int_{s}^{t}\left|f_{r}\right|_{W^{-2, p}} \mathrm{~d} r\left(1+\zeta^{-1}\right)\right. \\
\left.+\omega_{B}(s, t)^{3 \alpha}\|v\|_{L^{\infty}\left(L^{p}\right)}\left(1+\zeta+\zeta^{-1}\right)+\omega_{z}(s, t)^{z} \zeta^{-2}\right) .
\end{gathered}
$$

Observe further that in (3.28), $\eta$ must belong to the interval $(0,1)$ by definition of a family of smoothing operators, which will always be true if $(s, t) \in \Delta$ is chosen so that $\omega_{B}(s, t)<$ $L:=\zeta^{-1 / \alpha}$. If we fix $\zeta>0$ sufficiently large so that

$$
\frac{C_{\text {sewing }}(z) C_{J}}{\zeta^{2}} \leq \frac{1}{2}
$$

$C_{\text {sewing }}(z)$ being the constant of the Sewing Lemma, this leads to the smallness assumption:

$$
\omega_{B}(s, t) \leq L:=\left(C_{\text {sewing }}(z) C_{J}\right)^{-1 /(2 \alpha)} .
$$

Now, applying Proposition 3.1 and using (3.30), we see that for any $(s, t) \in \Delta$ with $\omega_{B}(s, t) \leq L$, it holds

$$
\left|v_{s t}^{\natural}\right|_{W^{-3, p}} \leq C_{z}\left(\omega_{B}(s, t)^{3 \alpha}\|v\|_{L^{\infty}\left(L^{p}\right)}+\omega_{B}(s, t)^{\alpha} \int_{s}^{t}\left|f_{r}\right|_{W^{-2, p}} \mathrm{~d} r\right)+\frac{1}{2} \omega_{z}(s, t)^{z},
$$

for some universal constant $C_{z}>0$. By the inequality $(a+b)^{\epsilon} \leq a^{\epsilon}+b^{\epsilon}$ for $a, b \geq 0$ and $\epsilon \in[0,1]$, we have

$$
\begin{aligned}
& \left|v_{s t}^{\natural}\right|_{W^{-3, p}}^{1 / z} \leq\left(C_{z}\right)^{1 / z}\left[\omega_{B}(s, t)^{3 \alpha / z}\|v\|_{L^{\infty}\left(L^{p}\right)}^{1 / z}\right. \\
& \left.+\omega_{B}(s, t)^{\alpha / z}\left(\int_{s}^{t}\left|f_{r}\right|_{W^{-2, p}} \mathrm{~d} r\right)^{1 / z}\right]+\frac{1}{2^{1 / z}} \omega_{z}(s, t)
\end{aligned}
$$

By [27, p.22], the above right hand side is a control, hence we infer from the property (3.25) that

$$
\begin{aligned}
& \omega_{z}(s, t) \leq\left(C_{z}\right)^{1 / z}\left[\omega_{B}(s, t)^{3 \alpha / z}\|v\|_{L^{\infty}\left(L^{p}\right)}^{1 / z}\right. \\
& \left.+\omega_{B}(s, t)^{\alpha / z}\left(\int_{s}^{t}\left|f_{r}\right|_{W^{-2, p}} \mathrm{~d} r\right)^{1 / z}\right]+\frac{1}{2^{1 / z}} \omega_{z}(s, t),
\end{aligned}
$$


which shows that for any $z \in(1,3 \alpha]$

$$
\begin{aligned}
\left|v_{s t}^{\natural}\right|_{W^{-3, p}}^{1 / z} \leq \omega_{z}(s, t) \leq\left(C_{z}\right)^{1 / z}(1 & \left.-\frac{1}{2^{1 / z}}\right)^{-1}\left[\omega_{B}(s, t)^{3 \alpha / z}\|v\|_{L^{\infty}\left(L^{p}\right)}^{1 / z}\right. \\
& \left.+\omega_{B}(s, t)^{\alpha / z}\left(\int_{s}^{t}\left|f_{r}\right|_{W^{-2, p}} \mathrm{~d} r\right)^{1 / z}\right] .
\end{aligned}
$$

Letting now $z=3 \alpha$ yields the inequality (3.22).

Proof of (3.23) Writing as before that $\delta v=\left(\mathrm{id}-J_{\eta}\right) \delta v+J_{\eta}\left(\int f \mathrm{~d} r+B^{1} v+B^{2} v+v^{\natural}\right)$, and then using (3.15)-(3.17), we have

$\llbracket \delta v \rrbracket_{-1}^{[\alpha]}(s, t) \lesssim\left(\eta+\omega_{B}(s, t)^{\alpha}+\frac{\omega_{B}(s, t)^{2 \alpha}}{\eta}\right)\|v\|_{L^{\infty}\left(s, t ; L^{p}\right)}+\frac{1}{\eta} \int_{s}^{t}\left|f_{r}\right|_{W^{-2, p}} \mathrm{~d} r+\frac{\llbracket v^{\natural} \rrbracket_{-3}^{[3 \alpha]}(s, t)}{\eta^{2}}$.

Combining with Proposition 3.3, this gives

$$
\begin{aligned}
\llbracket \delta v \rrbracket_{-1}^{[\alpha]}(s, t) \lesssim\left(\eta+\omega_{B}(s, t)^{\alpha}+\frac{\omega_{B}(s, t)^{2 \alpha}}{\eta}\right. & \left.+\frac{\omega_{B}(s, t)^{3 \alpha}}{\eta^{2}}\right)\|v\|_{L^{\infty}\left(s, t ; L^{p}\right)} \\
& +\left(\frac{1}{\eta}+\frac{\omega_{B}(s, t)^{\alpha}}{\eta^{2}}\right) \int_{s}^{t}\left|f_{r}\right|_{W^{-2, p}} \mathrm{~d} r .
\end{aligned}
$$

Upon choosing

$$
\eta:=\omega_{B}(s, t)^{\alpha}+\left(\int_{s}^{t}\left|f_{r}\right|_{W^{-2, p}} \mathrm{~d} r\right)^{\alpha}
$$

in (3.33), we obtain the estimate

$$
\begin{aligned}
& \left.\left|\delta v_{s t}\right|_{W^{-1, p}} \lesssim\left(\int_{s}^{t}\left|f_{r}\right|_{W^{-2, p}} \mathrm{~d} r\right)^{\alpha}+\omega_{B}(s, t)^{\alpha}\right)\|v\|_{L^{\infty}\left(s, t ; L^{p}\right)} \\
& +\left(\int_{s}^{t}\left|f_{r}\right|_{W^{-2, p}} \mathrm{~d} r\right)^{1-\alpha}+\omega_{B}(s, t)^{\alpha}\left(\int_{s}^{t}\left|f_{r}\right|_{W^{-2, p}} \mathrm{~d} r\right)^{1-2 \alpha}
\end{aligned}
$$

and the conclusion follows by the observation that $1-\alpha \geq \alpha$.

\section{The parabolic class $\mathcal{H}_{B}^{\alpha, p}$}

This section is devoted to the definition of a natural functional setting for rough partial differential equations of the form (2.24). In a second part, we will address the problem of obtaining an explicit equation for the product of two elements $u \in L^{\infty}\left(L^{p}\right)$ and $v \in$ $L^{\infty}\left(L^{p^{\prime}}\right)$, where $1 / p+1 / p^{\prime}=1$ and such that

$$
\mathrm{d} u=f \mathrm{~d} t+\mathrm{d} \mathbf{B} u
$$

while

$$
\mathrm{d} v=g \mathrm{~d} t+\mathrm{d} \mathbf{B} v
$$

on $[0, T] \times \mathbb{R}^{d}$, where $\mathbf{B}$ is a geometric, differential rough driver (here we consider $f$ and $g$ as given distributions). If $\mathbf{B}$ is "built over" a derivation-valued path, by which we mean that $B_{s t}^{1}=B_{t}-B_{s}$ for some $B_{t}=X_{t} \cdot \nabla$, one expects that $u v$ solves the problem

$$
\mathrm{d}(u v)=(u g+f v) \mathrm{d} t+\mathrm{d} \mathbf{B}(u v) .
$$

This indeed appears as a consequence of the Leibnitz-type identity $B_{t}(u v)=\left(B_{t} u\right) v+$ $u\left(B_{t} v\right)$, the geometricity of $\mathbf{B}$ and a formal application of [23, Proposition 7.6] (apply first the Itô formula on the square map, and then use polarization identities). For a more general 
geometric $\mathbf{B} \sim(X, \mathbb{L})$ (i.e. with a non-zero multiplicative term $\left.X_{s t}^{0}\right)$, a similar relation is expected, with the difference that $\mathbf{B}$ has to be "shifted" to a new object $\mathbf{B}^{(2)}$ of the same nature, but this time built over $X_{t} \cdot \nabla+2 X_{t}^{0}$. This fact will be made clear in the following paragraphs.

\subsection{A natural Banach space setting}

Let $p \in[1, \infty]$, fix a domain $U \subset \mathbb{R}^{d}$, and consider a $\mathcal{V}^{\alpha}$-differential rough driver $\mathbf{B}$ with $\alpha>1 / 3$. We define a space $\mathcal{H}_{B}^{\alpha, p}([0, T] \times U)$ as follows:

$$
\begin{aligned}
& \mathcal{H}_{B}^{\alpha, p}([0, T] \times U) \\
& :=\left\{\begin{aligned}
u \in L^{\infty}\left(L^{p}\right), & \text { such that }(u, u) \in \mathcal{D}_{B}^{\alpha, p}, \text { and there is } f \in L^{p}\left(W^{-1, p}(U)\right), \\
\text { satisfying } \mathrm{d} u=f \mathrm{~d} t & +\mathrm{d} \mathbf{B}(u, u), \text { and with the property that }
\end{aligned}\right. \\
& \|u\|_{\mathcal{H}_{B}^{\alpha, p}([0, T] \times U)}:=\|u\|_{L^{\infty}\left(L^{p}(U)\right)}+\|\nabla u\|_{L^{p}\left(L^{p}(U)\right)}+\|f\|_{L^{p}\left(W^{-1, p}(U)\right)} \\
& \left.+\|\delta u\|_{\mathcal{V}_{2}^{\alpha}\left(0, T ; W^{-1, p}(U)\right)}+\left\|R^{u}\right\|_{\mathcal{V}_{2}^{2 \alpha}\left(0, T ; W^{-2, p}(U)\right)}<\infty\right\},
\end{aligned}
$$

where we recall notation (3.12). As before, in the case when $U=\mathbb{R}^{d}$ we omit to indicate the domain, and we define local versions $\mathcal{H}_{B, \text { loc }}^{\alpha, p}$ of these spaces by the property

$$
\left.u \in \mathcal{H}_{B, \text { loc }}^{\alpha, p} \Leftrightarrow u\right|_{[0, T] \times K} \in \mathcal{H}_{B}^{\alpha, p}([0, T] \times K) \text { for every } K \subset \subset \mathbb{R}^{d} .
$$

One of the main interests in defining the above spaces is the next compactness-type result, which will be fundamental in the sequel.

Lemma $4.1\left(\mathcal{H}_{B}^{\alpha, p}\right.$-weak stability) Fix an open set $U \subset \mathbb{R}^{d}$, let $p \in[1, \infty]$ and consider a family $\{\boldsymbol{B}(n), n \in \mathbb{N}\} \cup\{\boldsymbol{B}\}$ of differential rough drivers such that $\rho_{\alpha}(\boldsymbol{B}(n), \boldsymbol{B}) \rightarrow 0$ where $\rho_{\alpha}$ is the distance introduced in (2.12). For each $n \geq 0$ consider $v(n) \in \mathcal{H}_{B(n)}^{\alpha, p}(U)$ and $f^{i}(n) \in L^{p}\left(L^{p}(U)\right), i=0, \ldots d$, such that

$$
\mathrm{d} v(n)=\left(\partial_{i} f^{i}(n)+f^{0}(n)\right) \mathrm{d} t+\mathrm{d} \boldsymbol{B}(n) v(n),
$$

weakly in $L^{p}$. Assume that the corresponding family is uniformly bounded in the sense that for every $n \geq 0$ :

for some constant $C>0$.

$$
\|v(n)\|_{\mathcal{H}_{B(n)}^{\alpha, p}(U)} \leq C,
$$

The following assertions are true.

(1) If $p>1$, there exists $n_{k} \nearrow \infty, k \rightarrow \infty$, some $f^{i} \in L^{p}\left(L^{p}\right), i=0, \ldots d$, and $v \in \mathcal{H}_{B}^{\alpha, p}$ so that

$$
\begin{aligned}
& v\left(n_{k}\right) \rightarrow v \quad \text { weakly-* in } L^{\infty}\left(0, T ; L^{p}(U)\right) \cap L^{2}\left(0, T ; W^{1, p}(U)\right), \\
& f\left(n_{k}\right) \rightarrow f \quad \text { weakly-* in } L^{p}\left(0, T ; W^{-1, p}(U)\right),
\end{aligned}
$$

while for any $\alpha^{\prime}<\alpha$ :

$$
\left(\delta v\left(n_{k}\right), R^{v\left(n_{k}\right)}\right) \rightarrow\left(\delta v, R^{v}\right) \quad \text { in } \mathcal{V}_{2}^{\alpha^{\prime}}\left(0, T ; W_{\mathrm{w}}^{-1, p}(U)\right) \times \mathcal{V}_{2}^{\alpha^{\prime}}\left(0, T ; W_{\mathrm{w}}^{-2, p}(U)\right)
$$

Moreover, $v$ satisfies

$$
\mathrm{d} v=\left(\partial_{i} f^{i}+f^{0}\right) \mathrm{d} t+\mathrm{d} \boldsymbol{B} v
$$

in the $L^{1}$-sense. 
(2) A similar conclusion holds for $p=1$ if the family $\{(v(n), f(n)), n \in \mathbb{N}\}$ is equiintegrable. Recall that $f(n)$ is said to be equi-integrable if it is bounded in $L^{1}$ and such that for any $\epsilon>0$, there exists $\delta_{\epsilon}>0$ and $\Omega_{\epsilon} \subset U$ with $\left|\Omega_{\epsilon}\right|<\infty$ so that uniformly in $n \geq 0$ :

$$
\iint_{[s, t] \times A}|f(n)| \mathrm{d} x \mathrm{~d} r \leq \epsilon
$$

for every $A \subset U$ measurable and $(s, t) \in \Delta$ such that $(t-s)|A| \leq \delta_{\epsilon}$, and

$$
\iint_{[0, T] \times(U \backslash \Omega)}|f(n)| \mathrm{d} x \mathrm{~d} r \leq \epsilon .
$$

Proof We first address the case $p>1$. In that case, the two first properties of (4.4) are just a consequence of Banach-Alaoglu Theorem, together with the definition of the spaces $\mathcal{H}_{B}^{\alpha, p}$. Concerning the last one, it relies on the following Aubin-Lions-type compactness result. The proof follows exactly the same steps as [39, Lemma A.2 \& Lemma A.3], and is therefore omitted.

Claim Let $\omega: \Delta \rightarrow \mathbb{R}_{+}$be a control function, let $p \in(1, \infty]$ and fix $L>0$. For $\kappa>0$, introduce the Banach space

$$
\begin{array}{r}
\mathfrak{X}^{\kappa}(\omega):=L^{\infty}\left(0, T ; L^{p}\right) \bigcap L^{p}\left(0, T ; L^{p}\right) \bigcap\left\{u \in \mathcal{V}^{\alpha}\left(0, T ; W^{-1, p}\right), \quad\left|\delta u_{s t}\right| \leq \omega(s, t)^{\kappa},\right. \\
\forall(s, t) \in \Delta \text { with } \omega(s, t) \leq L\},
\end{array}
$$

endowed with the norm

$$
\|u\|\left\|_{\kappa, \omega}:=\right\| u\left\|_{L^{\infty}\left(L^{p}\right)}+\right\| u \|_{L^{p}\left(W^{1, p}\right)}+\sup _{(s, t) \in \Delta} \frac{\left|\delta u_{s t}\right|_{W^{-1, p}}}{\omega(s, t)^{\kappa}} .
$$

Then,

$X^{\kappa}(\omega)$ is compactly embedded into

$$
L^{p}\left(0, T ; L_{\mathrm{loc}}^{p}\right) \cap L^{\infty}\left(0, T ; W_{\mathrm{loc}}^{-1, p}\right) \cap \mathcal{V}^{\kappa^{\prime}}\left(0, T ; W_{\mathrm{loc}}^{-2, p}\right) \text { for any } 0<\kappa^{\prime}<\kappa .(4.7)
$$

By definition of $\mathcal{H}_{B}^{\alpha, p}$, the norm of $v(n)$ in the controlled path space forms a uniformly bounded sequence. But thanks to Proposition 3.3, we also have the precise estimate

$$
\left|\delta v_{s t}(n)\right|_{W^{-1, p}} \leq C\left[\left(\int_{s}^{t}|f(n)|_{W^{-2, p}} \mathrm{~d} r\right)^{\alpha}+\omega_{B(n)}(s, t)^{\alpha}\right] \leq C^{\prime}(\alpha) \omega_{n}(s, t)^{\alpha}
$$

for any $(s, t) \in \Delta$ such that $\omega_{n}(s, t):=\omega_{B(n)}(s, t)+\int_{s}^{t}|f(n)|_{W^{-2, p}} \leq L$, where $L=L(\alpha)$ is independent of $n \in \mathbb{N}_{0}$. Though the estimate (4.8) suffers the fact that the control $\omega_{n}$ depends on $n \in \mathbb{N}_{0}$, we note that proceeding as in [47, Lemma 2.3], it is always possible to build a control $\varpi$ (depending on the whole sequence $\left\{\omega_{n}, n \in \mathbb{N}_{0}\right\}$ ) so that (4.8) holds with $\varpi$ for all $n \in \mathbb{N}_{0}$. For such $\varpi$, by definition of the space $\mathfrak{X}^{\alpha}(\varpi)$, we therefore obtain the uniform estimate:

$$
\left\|\delta v_{s t}(n)\right\|_{\alpha, \varpi} \leq C\|v(n)\|_{L^{\infty}\left(L^{p}\right)} \leq \widetilde{C},
$$

Hence property in (4.5) follows by the compact embedding (4.7), and the obvious inclusion $\mathfrak{X}^{\alpha}(\varpi) \subset \mathcal{V}^{\alpha}\left(0, T ; W^{-1, p}\right)$. 
Now, let $f(n) \in L^{p}\left(W^{-1, p}\right)$ such that $\mathrm{d} v(n)=f(n) \mathrm{d} t+\mathrm{d} \mathbf{B} v(n)$ for each $n \in \mathbb{N}$. Testing the equation against $\phi \in W^{3, p^{\prime}}(U)$ then yields for every $(s, t) \in \Delta$ :

$$
\left\langle\delta v_{s t}(n), \phi\right\rangle-\left\langle\left[B_{s t}^{1}(n)+B_{s t}^{2}(n)\right] v_{s}(n), \phi\right\rangle-\int_{s}^{t}\left\langle f_{r}(n), \phi\right\rangle \mathrm{d} r=\left\langle v_{s t}^{\natural}(n), \phi\right\rangle,
$$

where $v_{s t}^{\natural}(n) \in \mathcal{V}_{2}^{1+}\left(0, T ; W^{-3, p}(U)\right)$ denotes the remainder term.

We now show that $v$ belongs to $\mathcal{H}_{B}^{\alpha, p}$ and satisfies (4.6). In (4.9), the left hand side converges towards

$$
\left\langle\delta v_{s t}, \phi\right\rangle-\left\langle\left[B_{s t}^{1}+B_{s t}^{2}\right] v_{s}, \phi\right\rangle-\int_{s}^{t}\left\langle f_{r}, \phi\right\rangle \mathrm{d} r,
$$

for any $(s, t) \in \Delta$, as an obvious consequence of (4.4). Concerning the remainder term, it converges to some element $\left\langle v^{\natural}, \phi\right\rangle \in \mathcal{V}_{2}^{3 \alpha^{\prime}}(0, T ; \mathbb{R})$ for any $\alpha^{\prime}<\alpha$, as a consequence of (4.5) and the continuity part of the Sewing Lemma. Using the convergence of $\mathbf{B}(n)$ and Proposition 3.3, we see that $v^{\natural}$ defined above is actually an element of $\mathcal{V}_{2}^{3 \alpha}\left(0, T ; W^{-3, p}\right)$. By (3.23) and (3.24), one also obtains that $\left(\delta v, R^{v}\right)$ belongs to $\mathcal{V}_{2}^{\alpha}\left(W^{-1, p}\right) \times \mathcal{V}_{2}^{2 \alpha}\left(W^{-2, p}\right)$, showing that $v$ is indeed an element of $\mathcal{H}_{B}^{\alpha, p}$. This proves the first part.

Now, concerning the case $p=1$, as is well-known the Dunford-Pettis Theorem (see e.g. [1]) implies that a bounded family of $L^{1}$ is relatively weakly compact if and only if it is equi-integrable. Hence, the second assertion follows by the same argument as before, using a slight modification of the above compactness claim. We omit the details.

\subsection{Main result: product formula}

Let $u \in \mathcal{H}_{B}^{\alpha, p}$, and $v \in \mathcal{H}_{B}^{\alpha, p^{\prime}}$ with $1 / p+1 / p^{\prime}=1$. If $\mathbf{B}$ is geometric, it seems natural to expect that the pointwise product $u v$ belongs to $\mathcal{H}_{\tilde{B}}^{\alpha, 1}$ for some (possibly new) differential rough driver $\tilde{\mathbf{B}}$. The main result of this section gives a justification of this intuition, by showing a product formula for $u v$ (it could be alternatively thought of an "integration by parts" formula). By reiteration of the argument, a similar product formula will be shown on mononomials of bounded paths $u \in \mathcal{H}_{B}^{\alpha, 2}$, see Corollary 4.1.

In what follows, we consider a fixed open set $U \subset \mathbb{R}^{d}$.

Proposition 4.1 (Product formula, general case) Let $\boldsymbol{B}$ be a geometric, $\mathcal{V}^{\alpha}$-differential rough driver with $\alpha \in(1 / 3,1 / 2]$, fix $p, p^{\prime} \in[1, \infty]$ with $1 / p+1 / p^{\prime}=1$, and consider two elements $u, v \in \mathcal{H}_{B}^{\alpha, 1}(U)$ such that

$$
u \in L^{\infty}\left(0, T ; L^{p}(U)\right) \cap L^{p}\left(0, T ; W^{1, p}(U)\right)
$$

while

$$
v \in L^{\infty}\left(0, T ; L^{p^{\prime}}(U)\right) \cap L^{p^{\prime}}\left(0, T ; W^{1, p^{\prime}}(U)\right) .
$$

Let $f^{i}, g^{i} \in L^{1}\left(0, T ; L^{1}(U)\right), 0 \leq i \leq d$, such that on $[0, T] \times U$,

$$
\left[\begin{array}{l}
\mathrm{d} u=\left(\partial_{i} f^{i}+f^{0}\right) \mathrm{d} t+\mathrm{d} \boldsymbol{B} u, \\
\mathrm{~d} v=\left(\partial_{i} g^{i}+g^{0}\right) \mathrm{d} t+\mathrm{d} \boldsymbol{B} v, \quad \text { strongly in } L^{p}(U),
\end{array}\right.
$$

in the sense of Definition 2.4. Assume furthermore that for $i=0, \ldots, d$, the pointwise products $\partial_{i} u(\cdot) g^{i}(\cdot-a)$ and $f^{i}(\cdot-a) \partial_{i} v(\cdot)$ are in $L^{1}\left(0, T ; L^{1}(U)\right)$, for any $a \in \mathbb{R}^{d}$ with $|a| \leq 1$.

Then, the following holds: 
(i) The two-parameter mapping $\boldsymbol{B}^{(2)} \equiv\left(B^{(2), 1}, B^{(2), 2}\right)$, whose components are defined for $(s, t) \in \Delta$ as the differential operators

$$
\left\{\begin{array}{l}
B_{s t}^{(2), 1}:=B_{s t}^{1}+X_{s t}^{0}, \\
B_{s t}^{(2), 2}:=B_{s t}^{2}+X_{s t}^{0} X_{s t}^{i} \partial_{i}+\mathbb{L}_{s t}^{0}+\frac{3}{2}\left(X_{s t}^{0}\right)^{2},
\end{array}\right.
$$

is itself a geometric differential rough driver.

(ii) The pointwise product uv belongs to $\mathcal{H}_{B^{(2)}}^{\alpha, 1}(U)$ and is an $L^{1}(U)$-energy solution of

$$
\mathrm{d}(u v)=\left[u\left(\partial_{i} g^{i}+g^{0}\right)+\left(\partial_{i} f^{i}+f^{0}\right) v\right] \mathrm{d} t+\mathrm{d} \boldsymbol{B}^{(2)}(u v) .
$$

Regarding the definition of the spaces $\mathcal{H}_{B, \text { loc }}^{\alpha, p}$, we have the following immediate consequence of Proposition 4.1.

Corollary 4.1 (Product formula, transport case) Let $\boldsymbol{B} \sim(X, \mathbb{L})$ be as in Proposition 4.1 with $X^{0}=0$. Fix $p, p^{\prime} \in[1, \infty]$ so that $1 / p+1 / p^{\prime}=1$, and let $u \in \mathcal{H}_{B, \text { loc }}^{\alpha, p}$ be such that

$$
\mathrm{d} u=f \mathrm{~d} t+\mathrm{d} \boldsymbol{B} u \quad, \quad \text { on }[0, T] \times \mathbb{R}^{d},
$$

in the $L_{\text {loc }}^{p}$, strong sense, for some $f \in L^{p}\left(W^{-1, p}\right)$.

The following holds.

(I) Let $v \in \mathcal{H}_{B, \text { loc }}^{\alpha, p^{\prime}}$ be an $L_{\text {loc }}^{p^{\prime}}$-energy solution of

$$
\mathrm{d} v=g \mathrm{~d} t+\mathrm{d} \boldsymbol{B} v \quad \text { on }[0, T] \times \mathbb{R}^{d},
$$

with $g \in L^{p^{\prime}}\left(W^{-1, p^{\prime}}\right)$. Then, the product uv belongs to $\mathcal{H}_{B, \mathrm{loc}}^{\alpha, 1}$ and moreover $u v$ is an $L_{\text {loc }}^{1}$-energy solution of

$$
\mathrm{d}(u v)=(u g+f v) \mathrm{d} t+\mathrm{d} \boldsymbol{B}(u v) .
$$

(II) In the case where $p=2$ and $u$ belongs to $L_{\text {loc }}^{\infty}$, then for each $n \in \mathbb{N}_{0}$ we have $u^{n} \in \mathcal{H}_{B, \mathrm{loc}}^{\alpha, 1}$, and moreover:

$$
\mathrm{d}\left(u^{n}\right)=n u^{n-1} f \mathrm{~d} t+\mathrm{d} \boldsymbol{B}\left(u^{n}\right), \quad \text { on }[0, T] \times \mathbb{R}^{d}
$$

( $L_{\text {loc }}^{1}$ sense).

Remark 4.1 A similar conclusion as that of Corollary 4.1 holds when $\mathbf{B} \sim(X, \mathbb{L})$ with $X^{0} \neq 0$. In this case, it is easily seen by induction that for every $n \in \mathbb{N}$ :

$$
\mathrm{d}\left(u^{n}\right)=n u^{n-1} f \mathrm{~d} t+\mathrm{d} \mathbf{B}^{(n)}\left[u^{n}\right]
$$

in $L_{\text {loc }}^{1}$, where using the notation of Lemma $2.1, \mathbf{B}^{(n)}$ is the geometric differential rough driver defined as

$$
\left\{\begin{array}{l}
B_{s t}^{(n), 1}:=X_{s t}^{i} \partial_{i}+n X_{s t}^{0}, \\
B^{(n), 2}:=\frac{1}{2} X_{s t}^{i} X_{s t}^{j} \partial_{i j}+\left(\mathbb{L}_{s t}^{i}+n X_{s t}^{0} X_{s t}^{i}\right) \partial_{i}+n \mathbb{L}_{s t}^{0}+\frac{n^{2}}{2}\left(X_{s t}^{0}\right)^{2},
\end{array}\right.
$$

or making use of notation 2.1:

$$
\mathbf{B}^{(n)} \sim\left(\left(n X^{0}, X^{1}, \ldots, X^{d}\right) ;\left(n \mathbb{L}^{0}, \mathbb{L}^{1}, \ldots, \mathbb{L}^{d}\right)\right) .
$$


Before we proceed to the proof of Proposition 4.1, we need to introduce some additional notation. In what follows, we fix a bounded, open set $D \subset U$, such that

$$
\gamma:=1 \wedge \operatorname{dist}(D, \partial U)>0 .
$$

Notation 4.1 For $\epsilon \in(0,1]$ we will denote by $D_{\epsilon}$ the $\epsilon \gamma$-fattening of $D$, namely

$$
D_{\epsilon}:=\left\{x+\epsilon h \in \mathbb{R}^{d}, x \in D \text { and } h \in B_{\gamma}\right\} .
$$

For such $D$, we further define a set $\Omega_{\epsilon}^{D} \subset \mathbb{R}^{d} \times \mathbb{R}^{d}$ as follows:

$$
\Omega_{\epsilon}^{D}:=\left\{(x, y) \in U \times U, \frac{x+y}{2} \in D, \frac{x-y}{2} \in B_{\epsilon}\right\} .
$$

Notation 4.2 For $k \in I \subset \mathbb{Z}$ we define a linear, one-to-one transform $T_{\epsilon}$, by the formula

$$
T_{\epsilon} \Phi(x, y):=\frac{1}{(2 \epsilon)^{d}} \Phi\left(\frac{x+y}{2}+\frac{x-y}{2 \epsilon}, \frac{x+y}{2}-\frac{x-y}{2 \epsilon}\right),
$$

for all $\Phi \in W_{0}^{k, \infty}\left(\mathbb{R}^{d} \times \mathbb{R}^{d}\right)$. In particular, identifying $\Phi \in W_{0}^{k, \infty}\left(\Omega_{1}^{D}\right)$ with its extension by 0 outside its support, we have an isomorphism $T_{\epsilon}: W_{0}^{k, p}\left(\Omega_{1}^{D}\right) \rightarrow W_{0}^{k, p}\left(\Omega_{\epsilon}^{D}\right)$.

According to the terminology introduced in [19], any geometric differential rough driver is "renormalizable". This is the statement of the following Theorem, whose proof is rather technical and, for that reason, postponed in Appendix A.2.

Theorem 4.1 Let $\boldsymbol{B}$ be a geometric, differential rough driver with regularity $\alpha>1 / 3$.

Introduce the differential rough driver $\Gamma(\boldsymbol{B}) \equiv\left(\Gamma^{1}(\boldsymbol{B}), \Gamma^{2}(\boldsymbol{B})\right)$ given for every $(s, t) \in$ $\Delta$ by

$$
\left\{\begin{array}{l}
\Gamma_{s t}^{1}(\boldsymbol{B}):=B_{s t}^{1} \otimes \mathrm{id}+\mathrm{id} \otimes B_{s t}^{1} \\
\Gamma_{s t}^{2}(\boldsymbol{B}):=B_{s t}^{2} \otimes \mathrm{id}+B_{s t}^{1} \otimes B_{s t}^{1}+\mathrm{id} \otimes B_{s t}^{2}
\end{array}\right.
$$

(the fact that this is indeed a differential rough driver is elementary and hence left to the reader).

Then, for each $i=1,2$ and $k=-3+i, \ldots, 0$, the following uniform bound holds

$$
\left|T_{\epsilon}^{-1, *} \Gamma_{s t}^{i}(\boldsymbol{B}) T_{\epsilon}^{*}\right|_{\mathscr{L}\left(W^{1, k}\left(\Omega_{1}^{D}\right), W^{1, k-i}\left(\Omega_{1}^{D}\right)\right)} \leq C \omega_{B}(s, t)^{i \alpha}
$$

where $C>0$ denotes a constant which is independent of $\epsilon \in(0,1]$, while $\omega_{B}$ is the control introduced in Definition 2.1.

Before we proceed to the proof of the main result, let us observe that if $a \in W^{-k, p^{\prime}}$ and $b \in W^{k, p}$, then the product $a b$ has a well-defined meaning as an element of $a b \in W^{-1,1}$ (it suffices to write $a$ in terms of its antiderivatives, and to integrate by parts). Moreover, if $a, b$ are measurable functions (i.e. not distributions), then the adjoint of $T_{\epsilon}$ is given by the formula

$$
T_{\epsilon}^{*}[a(x) b(y)]=2^{-d} a\left(\frac{x+y}{2}+\epsilon \frac{x-y}{2}\right) b\left(\frac{x+y}{2}-\epsilon \frac{x-y}{2}\right) .
$$

Testing against $\Phi \in W_{0}^{k, \infty}\left(\Omega_{1}^{D}\right)$, and doing the change of variables $\left(x_{+}, x_{-}\right):=\chi(x, y) \equiv$ $\left(\frac{x+y}{2}, \frac{x-y}{2}\right)$, this gives the formula

$$
\left\langle T_{\epsilon}^{*} v, \Phi\right\rangle=\int_{B_{1} W^{-k, 1}(D)}\left\langle a\left(\cdot+\epsilon x_{-}\right) b\left(\cdot-\epsilon x_{-}\right), \Phi \circ \chi^{-1}\left(\cdot, x_{-}\right)\right\rangle_{W_{0}^{k, \infty}(D)} \mathrm{d} x_{-} .
$$


Now, in the general case where $a \in W^{-k, p^{\prime}}$ is a distribution, it is easily seen that (4.20) is still meaningful. This formula will be useful in the sequel.

We can now turn to the proof of the main result.

Proof of Proposition 4.1. Step 0: doubling of variables. In the sequel, we let for simplicity

$$
f:=\partial_{i} f^{i}+f^{0}, \quad g:=\partial_{i} g^{i}+g^{0},
$$

and denote by $u \otimes v$ the function of two variables

$$
(u \otimes v)_{t}(x, y):=u_{t}(x) v_{t}(y), \quad \text { for every }(x, y) \text { in } \Omega_{1}^{D} .
$$

For any $\epsilon \in(0,1)$ and $(s, t) \in \Delta$, we further introduce

$$
\left[\begin{array}{l}
(u \otimes v)_{s}^{\epsilon}:=T_{\epsilon}^{*}\left(\left.\left(u_{s} \otimes v_{s}\right)\right|_{\Omega_{\epsilon}^{D}}\right), \\
(f \otimes v+u \otimes g)_{s}^{\epsilon}:=T_{\epsilon}^{*}\left(\left.\left(f_{s} \otimes v_{s}+u_{s} \otimes g_{s}\right)\right|_{\Omega_{\epsilon}^{D}}\right), \\
\Gamma^{\epsilon}(\mathbf{B})=\left(\Gamma^{1, \epsilon}(\mathbf{B}), \Gamma^{2 \epsilon}(\mathbf{B})\right) \text { where }\left\{\begin{array}{l}
\Gamma_{s t}^{1, \epsilon}(\mathbf{B}):=T_{\epsilon}^{*} \Gamma_{s t}^{1}(\mathbf{B})\left(T_{\epsilon}^{*}\right)^{-1} \\
\Gamma_{s t}^{2, \epsilon}(\mathbf{B}):=T_{\epsilon}^{*} \Gamma_{s t}^{2}(\mathbf{B})\left(T_{\epsilon}^{*}\right)^{-1}
\end{array}\right.
\end{array}\right.
$$

Then, the following assertions are true.

(1) $(u \otimes v)^{\epsilon}$ belongs to $\mathcal{H}_{\Gamma(\mathbf{B})}^{\alpha, 1}\left(\Omega_{1}^{D}\right)$.

(2) the mapping $t \mapsto\left(f_{t} \otimes v_{t}+u_{t} \otimes g_{t}\right)^{\epsilon}$, is Bochner integrable in the space $W^{-1,1}\left(\Omega_{1}^{D}\right)$,

(3) $(u \otimes v)^{\epsilon}$ is an $L^{1}\left(\Omega_{1}^{D}\right)$-energy solution of the equation

$$
\mathrm{d}(u \otimes v)^{\epsilon}=(f \otimes v+u \otimes g)^{\epsilon} \mathrm{d} t+\mathrm{d} \Gamma^{\epsilon}(\mathbf{B})(u \otimes v)^{\epsilon} .
$$

The proof of the above properties is rather technical, but follows exactly the same pattern as that of [38, Section 5], hence we leave the details to the reader.

Step 1: uniform bound on the drift. If $\Phi \in W_{0}^{1, \infty}\left(\Omega_{1}^{D}\right)$ and $(s, t) \in \Delta$, we have by definition

$$
\left\langle\int_{s}^{t}\left(u_{r} \otimes g_{r}+f_{r} \otimes v_{r}\right)^{\epsilon} \mathrm{d} r, \Phi\right\rangle=\int_{s}^{t}\left\langle u_{r} \otimes g_{r}+f_{r} \otimes v_{r}, T_{\epsilon} \Phi\right\rangle \mathrm{d} r .
$$

Fix $r \in[s, t]$ such that $u \equiv u_{r}$ belongs to $W^{1, p}$, and let $\check{\Phi}\left(x_{+}, x_{-}\right):=\Phi \circ \chi^{-1}\left(x_{+}, x_{-}\right)=$ $\Phi\left(x_{+}+x_{-}, x_{+}-x_{-}\right)$. Making use of (4.20), we have for the first term in (4.25):

$$
\begin{aligned}
\left\langle u \otimes g, T_{\epsilon} \Phi\right\rangle= & \int_{B_{1} W^{-1, p^{\prime}}(D)}\left\langle g\left(\cdot-\epsilon x_{-}\right), u\left(\cdot+\epsilon x_{-}\right) \check{\Phi}\left(\cdot, x_{-}\right)\right\rangle_{W^{1, p}(D)} \mathrm{d} x_{-} \\
= & \iint_{B_{1} \times D}\left\{g^{i}\left(x_{+}+\epsilon x_{-}\right)(-1)^{i} \frac{\partial}{\partial x_{+}^{i}}\left[u\left(x_{+}+\epsilon x_{-}\right) \check{\Phi}\left(x_{+}, x_{-}\right)\right]\right. \\
& \left.+g^{0}\left(x_{+}+\epsilon x_{-}\right) u\left(x_{+}+\epsilon x_{-}\right) \check{\Phi}\left(x_{+}, x_{-}\right)\right\} \mathrm{d} x_{+} \mathrm{d} x_{-} .
\end{aligned}
$$


Hence, we have

$$
\begin{aligned}
\left\langle u \otimes g, T_{\epsilon} \Phi\right\rangle \leq & \iint_{B_{1} \times D}\left\{\left|g^{i}\left(x_{+}-\epsilon x_{-}\right)\right| \mid \partial_{i} u\left(x_{+}+\epsilon x_{-}\right)\right) \mid \\
& \left.\left.+\left|g^{0}\left(x_{+}-\epsilon x_{-}\right)\right| \mid u\left(x_{+}+\epsilon x_{-}\right)\right) \mid\right\}\left(|\check{\Phi}|+\left|\nabla_{+} \check{\Phi}\right|\right) \mathrm{d} x_{+} \mathrm{d} x_{-} \\
\leq & |\Phi|_{W^{1, \infty}} \int_{B_{1}} \mathrm{~d} x_{-} \int_{D+\epsilon x_{-}}\left\{\left|g^{i}\left(x_{+}-2 \epsilon x_{-}\right)\right|\left|\partial_{i} u\left(x_{+}\right)\right|\right. \\
& \left.\left.+\left|g^{0}\left(x_{+}-2 \epsilon x_{-}\right)\right| \mid u\left(x_{+}\right)\right) \mid\right\} \mathrm{d} x_{+} \\
\leq & |\Phi|_{W^{1, \infty}} \int_{B_{1}}\left(\left|g_{\left(\epsilon x_{-}\right)}^{i} \partial_{i} u\right|_{L^{1}\left(D_{\epsilon}\right)}+\left|g_{\left(\epsilon x_{-}\right)}^{0} u\right|_{L^{1}\left(D_{\epsilon}\right)}\right) \mathrm{d} x_{-},
\end{aligned}
$$

where for simplicity for $i=0, \ldots, d$, we denote by

$$
g_{\left(\epsilon x_{-}\right)}^{i}\left(x_{+}\right):=\left\{\begin{array}{l}
g^{i}\left(x_{+}-2 \epsilon x_{-}\right) \quad \text { if } x_{+}-2 \epsilon x_{-} \in D_{\epsilon} \\
0 \text { otherwise. }
\end{array}\right.
$$

(Note that, by assumption, the right hand side in (4.27) is finite.) Doing similar computations for the second term, and then integrating in time, we end up with the estimate

$$
\begin{aligned}
& \left|\int_{s}^{t}\left(u_{r} \otimes g_{r}+f_{r} \otimes v_{r}\right)^{\epsilon} \mathrm{d} r\right|_{W^{-1,1}\left(\Omega_{1}^{D}\right)} \\
& \leq \int_{B_{1}}\left(\left\|\partial_{i} u g_{\left(\epsilon x_{-}\right)}^{i}, u g_{\left(\epsilon x_{-}\right)}^{0}\right\|_{L^{1}\left(s, t ; L^{1}\left(D_{\epsilon}\right)\right)}+\left\|f_{\left(-\epsilon x_{-}\right)}^{i} \partial_{i} v, f_{\left(-\epsilon x_{-}\right)}^{0} v\right\|_{L^{1}\left(s, t ; L^{1}\left(D_{\epsilon}\right)\right)}\right) \mathrm{d} x_{-} \\
& =: \omega_{\mathscr{D}, D_{\epsilon}}(s, t),
\end{aligned}
$$

where we further observe that $\omega_{\mathscr{D}, D_{\epsilon}}$ is a control since positive linear combinations of controls are controls.

Step 2: convergence of the remainder term. For a.e. $r \in[s, t]$, it is straightforward to check the inequality

$$
\left|(u \otimes v)_{r}^{\epsilon}\right|_{L^{1}\left(\Omega_{1}^{D}\right)} \leq\left|D_{\epsilon}\right|\left|u_{r}\right|_{L^{p}\left(D_{\epsilon}\right)}\left|v_{r}\right|_{L^{p^{\prime}}\left(D_{\epsilon}\right)} .
$$

Therefore, by Theorem 4.1 together with Proposition 3.3 we obtain the following bound on the remainder $(u \otimes v)^{\epsilon, \natural}$ associated to (4.24):

$$
\begin{aligned}
& \left|(u \otimes v)_{s t}^{\epsilon, \natural}\right|_{W^{-3,1}\left(\Omega_{1}^{D}\right)} \leq C\left(\left|D_{\epsilon}\right|\left\|u_{r}\right\|_{L^{\infty}\left(L^{p}\left(D_{\epsilon}\right)\right)}\left\|v_{r}\right\|_{L^{\infty}\left(L^{p^{\prime}}\left(D_{\epsilon}\right)\right)} \omega_{B}(s, t)^{3 \alpha}\right. \\
& \left.+\omega_{\mathscr{D}, D_{\epsilon}}(s, t) \omega_{B}(s, t)^{\alpha}\right),
\end{aligned}
$$

for every $(s, t) \in \Delta$ such that $\omega_{B}(s, t) \leq L$ for some $L(\alpha)>0$, and every $\epsilon \in(0,1)$.

Fix

$$
\psi \in W_{0}^{3, \infty}\left(B_{1}\right), \quad \text { with } \quad \int_{B_{1}} \psi\left(x_{-}\right) \mathrm{d} x_{-}=1
$$

and for $(s, t)$ as above, denote by $\ell_{s t}^{\epsilon}$ the element of $W^{-3,1}(D)$ defined as

$$
\left\langle\ell_{s t}^{\epsilon}, \phi\right\rangle:=\left\langle(u \otimes v)_{s t}^{\epsilon, \natural},(\phi \otimes \psi) \circ \chi\right\rangle, \quad \text { for } \phi \in W_{0}^{3, \infty}(D) .
$$

By definition of $\ell^{\epsilon}$ and the estimate (4.29), we deduce that $\ell^{\epsilon}$ is uniformly bounded in $\mathcal{V}_{2, \text { loc }}^{3 \alpha}\left(0, T ; W_{\mathrm{w}}^{-3,1}(D)\right)$. Proceeding as in the proof of Lemma 4.1, we infer the existence 
of $\ell \in \mathcal{V}_{2, \text { loc }}^{3 \alpha}\left(0, T ;\left(W_{0}^{3, \infty}(D)\right)^{*}\right)$ and $\epsilon_{n} \searrow 0$ such that for any $\alpha^{\prime}<\alpha$ and every $\phi \epsilon$ $W_{0}^{3, \infty}(D)$

$$
\left\langle\ell^{\epsilon_{n}}, \phi\right\rangle \rightarrow\langle\ell, \phi\rangle \quad \text { in } \mathcal{V}_{2, \text { loc }}^{3 \alpha^{\prime}}(0, T ; \mathbb{R})
$$

which in particular implies convergence in the $C(\Delta ; \mathbb{R})$-sense.

It remains to show that $\ell_{s t}$ belongs to $W^{-3,1}(D)$ for any $(s, t) \in \Delta$. In (4.29), substitute $D$ with any $K \subset D$ and then take the limit as $\epsilon \rightarrow 0$. This yields

$$
\begin{aligned}
& \left|\ell_{s t}\right|_{\left(W_{0}^{3, \infty}(K)\right)^{*}} \leq C\left[|K|\|u\|_{L^{\infty}\left(L^{p}(K)\right)}\|v\|_{L^{\infty}\left(L^{\left.p^{\prime}(K)\right)}\right.} \omega_{B}(s, t)^{3 \alpha}\right. \\
& \left.+\left(\left\|\partial_{i} u g^{i}, u g^{0}\right\|_{L^{1}\left(s, t ; L^{1}(K)\right)}+\left\|f^{i} \partial_{i} v, f^{0} v\right\|_{L^{1}\left(s, t ; L^{1}(K)\right)}\right) \omega_{B}(s, t)^{\alpha}\right] .
\end{aligned}
$$

This implies that $\left|\ell_{s t}\right|_{\left(W_{0}^{3, \infty}(K)\right)^{*}}$ goes to 0 , as $|K| \rightarrow 0$. As is well-known (see e.g. [7, Proposition 4.4.2 p. $263 \&$ Proposition 1.3 .3 p. 9]) this implies that $\ell$ is an element of the subspace $W^{-3,1}(D)$. This proves the claimed property.

Step 3: passage to the limit in the equation Fix any $\phi \in W^{3, \infty}(U)$ with compact support in $D$, and test (4.24) against

$$
\Phi(x, y):=\phi\left(\frac{x+y}{2}\right) \psi\left(\frac{x-y}{2}\right), \quad(x, y) \in \Omega_{1}^{D},
$$

which is indeed an element of $W^{3, \infty}\left(\Omega_{1}^{D}\right)$. Observe furthermore that $T_{\epsilon} \Phi(x, y)=$ $\phi\left(\frac{x+y}{2}\right) \psi_{\epsilon}(x-y)$ where

$$
\psi_{\epsilon}(\cdot)=\psi_{\epsilon}(\cdot / 2)(2 \epsilon)^{-d}
$$

approximates the identity.

Hence, using Theorem 4.1 and dominated convergence, we find that

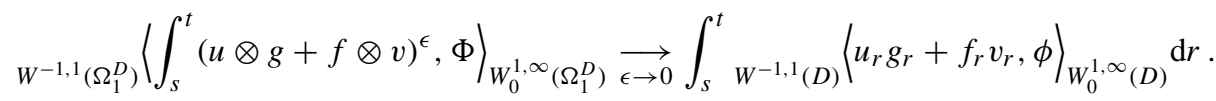

For the terms involving $\Gamma(\mathbf{B})$, we first note that by Lemma 2.1, the following Leibniz-type formulas are satisfied: for every $a, b \in C^{\infty}$ it holds

$$
\left\{\begin{array}{l}
B_{s t}^{1}(a b)=\left(B_{s t}^{1} a\right) b+a\left(B_{s t}^{1} b\right)-X_{s t}^{0} a b, \\
B_{s t}^{2}(a b)=\left(B_{s t}^{2} a\right) b+\left(B_{s t}^{1} a\right)\left(B_{s t}^{1} b\right)+a\left(B_{s t}^{2} b\right)-X_{s t}^{i} X_{s t}^{0} \partial_{i}(a b)-\left(\mathbb{L}_{s t}^{0}+\frac{3}{2}\left(X_{s t}^{0}\right)^{2}\right) a b .
\end{array}\right.
$$

Now, using dominated convergence and (4.33) yields for the first term

$$
\begin{aligned}
& W^{-1,1}\left(\Omega_{1}^{D}\right) \\
\longrightarrow & \left\langle\Gamma_{s t}^{1, \epsilon}(\mathbf{B})(u \otimes v)_{s}^{\epsilon}, \Phi\right\rangle_{W_{0}^{1, \infty}\left(\Omega_{1}^{D}\right)} \\
& { }_{W^{-1,1}(D)}\left\langle\left(B_{s t}^{1} u_{s}\right) v_{s}+v_{s} B_{s t}^{1} u_{s}, \phi\right\rangle_{W_{0}^{1, \infty}(D)} \\
= & \left\langle\left(B_{s t}^{1}+X_{s t}^{0}\right)(u v), \phi\right\rangle=\left\langle B_{s t}^{(2), 1}(u v), \phi\right\rangle,
\end{aligned}
$$

by definition of $\mathbf{B}^{(2), 1}$. Similarly, using the second equation in (4.33), it is easily seen that

$$
\begin{aligned}
& W^{-2,1}\left(\Omega_{1}^{D}\right) \\
& \underset{\epsilon \rightarrow 0}{\epsilon}\left\langle\left(\Gamma_{s t}^{2, \epsilon}(\mathbf{B})(u \otimes v)_{s}^{\epsilon}, \Phi\right\rangle_{W_{0}^{2, \infty}\left(\Omega_{1}^{D}\right)}\right) \\
= & \left.\left\langle\left(B_{s t}^{2}+X_{s t}^{0} X_{s t}^{i} \partial_{i}+\mathbb{L}_{s t}^{0}+\frac{3}{2}\left(X_{s t}^{1} u_{s}\right)\left(B_{s t}^{1} v_{s}\right)+u_{s}\left(B_{s t}^{2} v_{s}\right), \phi\right\rangle\right), \phi\right\rangle=\left\langle B_{s t}^{(2), 2}(u v), \phi\right\rangle .
\end{aligned}
$$


Finally, we have $\left\langle\delta(u \otimes v)_{s t}^{\epsilon}, \Phi\right\rangle \rightarrow_{\epsilon \rightarrow 0}\left\langle\delta(u v)_{s t}, \phi\right\rangle$, and hence using the previous step:

$$
\left\langle\delta(u v)_{s t}, \phi\right\rangle=\int_{s}^{t}\langle u g+f v, \phi\rangle \mathrm{d} r+\left\langle\left(B_{s t}^{(2), 1}+B_{s t}^{(2), 2}\right)(u v), \phi\right\rangle+\left\langle\ell_{s t}, \phi\right\rangle,
$$

for every $(s, t) \in$ such that $\omega_{B}(s, t) \leq L$. The (4.35) holds for any open and bounded $D \subset U$ with positive distance from $U$. Thus, it remains true for $U$ itself, which shows that $u v$ is an $L^{1}(U)$-weak solution of (4.11).

It remains to show that $\mathbf{B}^{(2)}$ is a differential rough driver, for which it suffices to check that Chen's relations (2.10) hold. But these are an immediate consequence of Lemma 2.1 and the linearity of $\delta$, since:

$$
\begin{aligned}
\delta B_{s \theta t}^{(2), 2} & \equiv \delta\left(B^{2}+X^{0} X^{i} \partial_{i}+\mathbb{L}^{0}+\frac{3}{2}\left(X^{0}\right)^{2}\right)_{s \theta t} \\
& =B_{\theta t}^{1} \circ B_{s \theta}^{1}+\left(X_{\theta t}^{0} X_{s \theta}^{i}+X_{s \theta}^{0} X_{\theta t}^{i}\right) \partial_{i}+X_{\theta t}^{i} \partial_{i} X_{s \theta}^{0}+3 X_{\theta t}^{0} X_{s \theta}^{0} \\
& =\left(B_{\theta t}^{1}+X_{\theta t}^{0}\right) \circ\left(B_{s \theta}^{1}+X_{s \theta}^{0}\right)=B_{\theta t}^{(2), 1} \circ B_{s \theta}^{(2), 1},
\end{aligned}
$$

for $(s, \theta, t) \in \Delta_{2}$. This shows that $\mathbf{B}^{(2)}$ is a differential rough driver. Moreover, $\mathbf{B}^{(2)}$ is obviously geometric since $\mathbf{B}$ is.

Finally, thanks to Proposition 3.3, we further see that $u v$ is controlled by $B^{(2)}$, and thus it belongs to $\mathcal{H}_{B^{(2)}, \text { loc }}^{\alpha, 1}$. This achieves the proof of (ii) and the proposition.

\section{Parabolic equations with free terms: proof of Theorem 2.1}

In this section we investigate existence, uniqueness and stability for parabolic rough partial differential equations of the form

$$
\begin{array}{r}
\mathrm{d} u=(A u+f) \mathrm{d} t+\mathrm{d} \mathbf{B} u, \quad \text { on }[0, T] \times \mathbb{R}^{d} \\
u_{0} \in L^{2}\left(\mathbb{R}^{d}\right),
\end{array}
$$

where $f$ belongs to the space $L^{2}\left(0, T ; H^{-1}\right)$. This completes the case treated in [38], where a more general elliptic operator $A$ was considered, but where the assumptions on $\mathbf{B}$ were more restrictive. For the reader's convenience, we now restate Theorem 2.1.

Theorem 5.1 Let $f \in L^{2}\left(0, T ; H^{-1}\right)$, fix $u_{0} \in L^{2}$ and consider a geometric, differential rough driver $\boldsymbol{B}$ with regularity $\alpha>1 / 3$. There exists a unique $L^{2}$-energy solution $u=$ $u\left(u_{0}, f ; \boldsymbol{B}\right)$ to $(5.1)$, and it belongs to the space $\mathcal{H}_{B}^{\alpha, 2}\left(\mathbb{R}^{d}\right)$.

Moreover, the solution map is continuous in the following sense

(C1) for every $\left(u_{0}, f\right) \in L^{2} \times L^{2}\left(H^{-1}\right)$, the map $\boldsymbol{B} \mapsto u\left(u_{0}, f ; \boldsymbol{B}\right)$ is continuous in the following sense: for any sequence $\left\{\boldsymbol{B}(n), n \in \mathbb{N}_{0}\right\}$ of geometric differential rough drivers such that $\rho_{\alpha}(\boldsymbol{B}(n), \boldsymbol{B}) \rightarrow 0$, denoting by $u(n)$ the solution of (5.1) obtained with $\boldsymbol{B}$ being replaced by $\boldsymbol{B}(n)$, it holds

$$
u(n) \rightarrow u \quad \text { weakly-* in } L^{\infty}\left(0, T ; L^{p}(U)\right) \cap L^{2}\left(0, T ; W^{1, p}(U)\right),
$$

and for any $\alpha^{\prime}<\alpha$ :

$$
\left(\delta u(n), R^{u(n)}\right) \rightarrow\left(\delta u, R^{u}\right) \quad \text { in } \mathcal{V}_{2}^{\alpha^{\prime}}\left(0, T ; W_{\mathrm{w}}^{-1, p}(U)\right) \times \mathcal{V}_{2}^{\alpha^{\prime}}\left(0, T ; W_{\mathrm{w}}^{-2, p}(U)\right) .
$$

(C2) for $\boldsymbol{B}$ fixed the map $u(\cdot, \cdot ; \boldsymbol{B}): L^{2} \times L^{2}\left(H^{-1}\right) \rightarrow \mathcal{H}_{B}^{\alpha, 2}$ is continuous, with respect to the strong topologies. 
Note that the above result obviously implies Theorem 2.1. Its proof essentially follows the lines of [38] but since our assumptions on $\mathbf{B}$ are more general, we provide a complete proof.

Proof of Theorem 5.1 Consider an $L^{2}$-energy solution $u \in \mathcal{H}_{B}^{\alpha, 2}$ of the (5.1). Applying Proposition 4.1 with $u=v$, we have that $u^{2} \in \mathcal{H}_{B^{(2)}}^{\alpha, 1}$ where $\mathbf{B}^{(2)}$ is the shifted differential rough driver defined in (4.10). Moreover, $u^{2}$ solves in the $L^{1}$-sense:

$$
\mathrm{d} u^{2}=2 u(A u+f) \mathrm{d} t+\mathrm{d} \mathbf{B}^{(2)}\left(u^{2}\right) .
$$

We want to test against $\phi=1$, and then apply Rough Gronwall, but for this we need first an estimate on $u^{2, \natural}$, which itself follows from Proposition 3.3, together with the estimate on the drift. The analysis of the linear part of the drift leads to the estimate:

$$
\left|\int_{s}^{t}(u A u) \mathrm{d} r\right|_{W^{-1,1}} \leq \lambda^{-1}\left(\|\nabla u\|_{L^{2}\left(s, t ; L^{2}\right)}^{2}+\|u \nabla u\|_{L^{1}\left(s, t ; L^{1}\right)}\right)
$$

whereas for the free term, considering anti-derivatives, we find

$$
\int_{s}^{t}|u f|_{W^{-1,1}} \mathrm{~d} r \leq\left(\|u\|_{L^{2}\left(s, t ; L^{2}\right)}+\|\nabla u\|_{L^{2}\left(s, t ; L^{2}\right)}\right)\|f\|_{L^{2}\left(s, t ; H^{-1}\right)} .
$$

The proof is then divided into 3 steps.

Step 1: Energy inequality and application to uniqueness. Letting $\omega_{\mathscr{D}}(s, t)$ be the sum of the right hand sides in (5.3) and (5.4), one can then apply Proposition 3.3 to obtain

$$
\left|u_{s t}^{2, \natural}\right|_{W^{-3,1}} \leq C\left(\omega_{B}(s, t)^{\alpha} \omega_{\mathscr{D}}(s, t)+\|u\|_{L^{\infty}\left(s, t ; L^{2}\right)}^{2} \omega_{B}(s, t)^{3 \alpha}\right) .
$$

for every $(s, t) \in \Delta$ with $\omega_{B}(s, t) \leq L$ for some absolute constant $L>0$.

Next, consider $f=\partial_{i} \mathbf{f}^{i}+\mathbf{f}^{0}$ where $\mathbf{f}^{i} \in L^{2}, i=0, \ldots, d$. One can take $\phi=1 \in W^{3, \infty}$ in (5.2), so that by Assumption 2.1 it holds for every $s, t$ as above:

$$
\begin{aligned}
\delta E_{s t}:= & \delta\left(|u|_{L^{2}}^{2}\right)_{s t}+\int_{s}^{t}\left|\nabla u_{r}\right|_{L^{2}}^{2} \mathrm{~d} r \\
& \lesssim \lambda \iint_{[s, t] \times \mathbb{R}^{d}}-\partial_{i} u_{r}(x) \mathbf{f}_{r}^{i}(x) \mathrm{d} x \mathrm{~d} r+\left\langle\left(B_{s t}^{(2), 1}+B_{s t}^{(2), 2}\right) u_{s}^{2}+u_{s t}^{2, \natural}, 1\right\rangle \\
& \lesssim \lambda\|\nabla u\|_{L^{2}\left(s, t ; L^{2}\right)}\|\mathbf{f}\|_{L^{2}\left(s, t ; L^{2}\right)}+\left|u_{s}\right|_{L^{2}}^{2}\left(\omega_{B}(s, t)^{\alpha}\right. \\
& \left.+\omega_{B}(s, t)^{2 \alpha}\right)+\left|u_{s t}^{2, \natural}\right|_{W^{-3,1}}, \\
& \lesssim \lambda\|\nabla u\|_{L^{2}\left(s, t ; L^{2}\right)}\|\mathbf{f}\|_{L^{2}\left(s, t ; L^{2}\right)}+\left(\omega_{B}(s, t)^{\alpha}\right. \\
& \left.+\omega_{B}(s, t)^{\alpha}+\omega_{B}(s, t)^{3 \alpha}\right) \sup _{r \in[s, t]} E_{r} \\
& +\omega_{B}(s, t)^{\alpha}\|f\|_{L^{2}\left(s, t ; H^{-1}\right)}\left(\|\nabla u\|_{L^{2}\left(s, t ; L^{2}\right)}+\|u\|_{L^{2}\left(s, t ; L^{2}\right)}\right)
\end{aligned}
$$

Making use of Young Inequality

$$
\|\nabla u\|_{L^{2}\left(s, t ; L^{2}\right)}\|\mathbf{f}\|_{L^{2}\left(s, t ; L^{2}\right)} \leq \frac{\epsilon}{2}\|\nabla u\|_{L^{2}\left(s, t ; L^{2}\right)}^{2}+\frac{1}{2 \epsilon}\|\mathbf{f}\|_{L^{2}\left(s, t ; L^{2}\right)}^{2}
$$

for $\epsilon(\lambda)>0$ sufficiently small, the first term in the right hand side can be absorbed to the left. Hence, taking $L$ smaller if necessary, we infer that for any $(s, t) \in \Delta$ with $\omega_{B}(s, t) \leq L$, it holds the incremental inequality

$$
\delta E_{s t} \leq \omega_{B}(s, t)^{\alpha}\left(\sup _{r \in[s, t]} E_{r}\right)+\|f\|_{L^{2}\left(s, t ; H^{-1}\right)}^{2} .
$$


By Lemma 3.1, we deduce the estimate

$$
\begin{aligned}
\|u\|_{L^{\infty}\left(0, T ; L^{2}\right)}^{2}+ & \|\nabla u\|_{L^{2}\left(0, T ; L^{2}\right)}^{2} \\
& \leq C(\lambda) \exp \left\{\frac{\omega_{B}(0, T)}{\tau_{\alpha, L}}\right\}\left[\left|u_{0}\right|_{L^{2}}^{2}+\|f\|_{L^{2}\left(0, T ; H^{-1}\right)}^{2}\right] .
\end{aligned}
$$

The uniqueness is now straightforward, because the difference $v \equiv u_{1}-u_{2}$ of two $L^{2}$-energy solutions to (5.1) ought to be itself an $L^{2}$-energy solution of (5.1), with $f=0$ and $v_{0}=0$, hence yielding from (5.6) that $v=0$.

Step 2: Existence. Existence and continuity rely mostly on the stability result shown in Lemma 4.1, together with the fact that $\mathbf{B}$ is geometric.

Consider a sequence $\mathbf{B}(n) \rightarrow \mathbf{B}$ as in Definition 2.2. By standard results on parabolic equations, there exists a unique $u(n)$ in the energy space $L^{\infty}\left(L^{2}\right) \cap$ $L^{2}\left(H^{1}\right)$, solving (5.1) in the sense of distributions. Using moreover the fact that $\mathbf{B}(n)=S_{2}(B(n))$, it is easily deduced from (5.1) that $u(n)$ is an $L^{2}$-energy solution of (5.1), in the sense of Definition 2.5. Consequently, the previous analysis shows that we have a uniform bound

$$
\|u(n)\|_{L^{\infty}\left(0, T ; L^{2}\right)}^{2}+\|\nabla u(n)\|_{L^{2}\left(0, T ; H^{1}\right)}^{2} \leq C\left(\lambda,\|f\|_{L^{2}\left(0, T ; H^{-1}\right)},\left|u_{0}\right|_{L^{2}}, T\right) .
$$

As a consequence of this bound and Proposition 3.3, we also obtain the uniform estimate

$$
\|u(n)\|_{\mathcal{H}_{B(n)}^{\alpha, 2}} \leq C^{\prime},
$$

for another such constant $C^{\prime}$. By Lemma 4.1 we see that $\{u(n), n \in \mathbb{N}\}$ has a (possibly non-unique) limit point $u \in \mathcal{H}_{B}^{\alpha, 2}$ such that the weak-type convergences of (4.4)-(4.5) hold, up to some subsequence $u\left(n_{k}\right) n_{k} \nearrow \infty$. In particular, each of the terms in the equation on $u\left(n_{k}\right)$ converges to the expected quantities associated to the limit $u$. This shows the claimed existence.

Step 3: Stability. We can now repeat the argument of Step 3 with any sequence $\mathbf{B}(n)$ of geometric, differential rough drivers (not necessarily defined as canonical lifts). This will imply the convergence of a subsequence $u\left(n_{k}\right) \rightarrow u$, in the sense of (4.4) and (4.5). From the uniqueness part, there can be at most one such limit $u$, and therefore every subsequence of $u(n)$ converges to $u$. This implies the convergence of the full sequence, and the claimed continuity $(\mathrm{C} 1)$.

To show (C2), note that if $u$ and $v$ are $L^{2}$-energy solutions of

$$
\begin{array}{ll}
\mathrm{d} u=(A u+f) \mathrm{d} t+\mathrm{d} \mathbf{B} u, & u_{0}=u^{0}, \\
\mathrm{~d} v=(A v+g) \mathrm{d} t+\mathrm{d} \mathbf{B} v, & v_{0}=v^{0},
\end{array}
$$

where $u^{0}, v^{0} \in L^{2}$, and $f, g \in L^{2}\left(H^{-1}\right)$, then $w:=u-v$ solves the problem

$$
\mathrm{d} w=(A w+f-g) \mathrm{d} t+\mathrm{d} \mathbf{B} w, \quad w_{0}=u^{0}-v^{0} .
$$

Therefore, the strong continuity of the solution map with respect to $\left(u_{0}, f\right)$ follows from the estimate (5.6), together with Proposition 3.3.

\section{Local boundedness of solutions}

In this section, we take a step further by investigating the boundedness, away from $t=0$ and on any compact set of the space variable, for solutions of parabolic RPDEs of previous 
form, namely

$$
\begin{array}{r}
\mathrm{d} u=(A u+f) \mathrm{d} t+\mathrm{d} \mathbf{B} u, \quad \text { in }[0, T] \times \mathbb{R}^{d}, \\
u_{0} \in L^{2}\left(\mathbb{R}^{d}\right),
\end{array}
$$

where the free term $f$ will be subject to additional conditions, see Assumption 6.1, and $A$ fulfills Assumption 2.1.

First, let us recall a classical interpolation inequality, the proof of which can be found in [46].

Proposition 6.1 For each $f$ in the space $L^{\infty}\left(0, T ; L^{2}\right) \cap L^{2}\left(0, T ; W^{1,2}\right), f$ belongs to $L^{\rho}\left(0, T ; L^{\sigma}\right)$ for every $\rho, \sigma$ such that

$$
\frac{1}{\rho}+\frac{d}{2 \sigma} \geq \frac{d}{4} \quad \text { and }\left\{\begin{array}{lll}
\rho \in[2, \infty], & \sigma \in\left[2, \frac{2 d}{d-2}\right] \text { for } d>2 \\
\rho \in(2, \infty], & \sigma \in[2, \infty) \text { for } d=2 \\
\rho \in[4, \infty], & \sigma \in[2, \infty] \text { for } d=1 .
\end{array}\right.
$$

In addition, there exists a constant $C_{\rho, \sigma}>0$ (not depending on $f$ in the above space) such that

$$
\|f\|_{L^{\rho}\left(0, T ; L^{\sigma}\right)} \leq C_{\rho, \sigma}\left(\|\nabla f\|_{L^{2}\left(0, T ; L^{2}\right)}+\underset{r \in[0, T]}{\operatorname{ess} \sup }\left|f_{r}\right|_{L^{2}}\right) .
$$

As an immediate consequence of (6.3), it can be checked that whenever $r, q \in[1, \infty]$ are numbers satisfying

$$
\frac{1}{r}+\frac{d}{2 q} \leq 1
$$

then it holds the inequality

$$
\|u\|_{L^{\frac{2 r}{r-1}\left(L^{\frac{2 q}{q-1}}\right)}} \leq C_{r, q}\|u\|_{L^{\infty}\left(L^{2}\right) \cap L^{2}\left(H^{1}\right)} .
$$

\subsection{Moser Iteration}

Recall the basic idea of Moser's iteration. If $u \in L^{\infty}\left(0, T ; L^{2}\right) \cap L^{2}\left(0, T ; H^{1}\right)$ solves a parabolic equation of the form (6.1) where the coefficients are smooth enough, the new unknown $|u|^{\varkappa}$ for $\varkappa \geq 2$ is, roughly speaking, solution of a similar equation. By a slight modification of the arguments of the Section 5 , it is possible thanks to the above interpolation inequality to find suitable moment bounds for $v:=|u|^{\varkappa / 2}$, the value of which depend on similar moments, but for a lower exponent. Thanks to (6.3), we will then obtain a recursive relation between these quantities, which will take the form of the following inequality

$$
\Phi_{n+1} \leq \gamma \tau^{n} \Phi_{n}^{1+\epsilon}, \quad \text { for any } n \geq 0,
$$

where $\epsilon, \gamma, \tau>0$ are constants. It is worth noting that the above inequality is non-linear, and that the coefficent $\tau^{n}$ will blow up unless $\tau$ is smaller than one. Hence, an upper bound of $\Phi_{n}$ may blow-up as well when $n \rightarrow \infty$. However, the next result shows that this explosion is "not too strong" for our purposes. The proof is immediate by induction, and therefore omitted.

Lemma 6.1 (Recursive estimate) Assume that we are given a sequence of non-negative numbers $\Phi_{n}, n \geq 0$, and constants $\epsilon, \gamma, \tau>0$ such that (6.6) holds. Then, the following 
estimate is true: for any $n \geq 0$ we have

$$
\Phi_{n} \leq \gamma^{\frac{(1+\epsilon)^{n}-1}{\epsilon}} \tau^{\frac{(1+\epsilon)^{n}-1}{\epsilon^{2}}-\frac{n}{\epsilon}} \Phi_{0}^{(1+\epsilon)^{n}} .
$$

Now, a classical result states that

$$
|f|_{L^{\varkappa}(X, \mathcal{M}, \mu)} \underset{\varkappa \rightarrow \infty}{\rightarrow}|f|_{L^{\infty}(X, \mathcal{M}, \mu)},
$$

for any $\sigma$-finite measure space $(X, \mathcal{M}, \mu)$ and every $f \in L^{\infty}$ such that $f \in L^{q}$ for some $q \in[1, \infty)$. Using that result and the fact that $\Phi_{n}$ will be taken below to be an appropriate sequence of moments with diverging exponents, we will be able to obtain an a priori estimate for the $L^{\infty}$-norm of $u$. This will prove the boundedness of solutions.

We need now to specify our conditions on $f$.

\section{Assumption 6.1 We assume that}

$$
f \in \mathscr{M}:=L^{r}\left(0, T ; W^{-1, q}\right) \cap L^{2 r}\left(0, T ; W^{-1,2 q}\right) \cap L^{1}\left(0, T ; W^{-1,1}\right) \cap L^{2}\left(0, T ; H^{-1}\right),
$$

where the exponents $r \in(1, \infty]$ and $q \in\left(1 \vee \frac{d}{2}, \infty\right)$ are subject to the conditions

$$
\frac{1}{r}+\frac{d}{2 q}<1 \text {. }
$$

Using Sobolev embeddings, it is easily checked that Assumption 6.1 is fulfilled for $f$ satisfying the assumptions of Theorem 2.3, i.e. $f \in L^{r}\left(0, T ; L^{q}\right)$, where $r$ and $q$ verify the condition (6.8). Hence, the following result implies Theorem 2.3.

Proposition 6.2 Let Assumption 6.1 hold, suppose that $u_{0} \in L^{2}$, and assume that $u$ is the solution of (6.1) given by Theorem 5.1. Then, the essential supremum of $u$ is bounded on each compact subset of $(0, T] \times \mathbb{R}^{d}$.

In addition, for any $Q \subset \subset(0, T] \times \mathbb{R}^{d}$, it holds the estimate

$$
\|u\|_{L^{\infty}(Q)} \leq C\left(Q,\left|u_{0}\right|_{L^{2}}, \lambda,\|f\|_{\mathscr{M}}, \omega_{B}, \alpha, r, q\right),
$$

for a constant depending only on the indicated quantities.

\subsection{The recursive estimate}

Our purpose in the present paragraph is to show that a suitable sequence $\left\{\Phi_{n}, n \in \mathbb{N}_{0}\right\}$ can be defined, so that Lemma 6.1 will be applicable and provide the claimed $L_{\text {loc }}^{\infty}$ estimate. Consider $u \in \mathcal{H}_{B}^{\alpha, 2} \cap L^{\infty}, L^{2}$-energy solution of (2.24), and let $\varkappa \geq 2$. Assuming for the moment that the conclusions of Theorem 2.4 are true, we have in the $L^{1}$-sense:

$$
\delta|u|_{s t}^{\varkappa}=\int_{s}^{t} \varkappa u_{r}\left|u_{r}\right|^{\varkappa-2}\left(A_{r} u_{r}+f_{r}\right) d r+\left(B_{s t}^{1}+B_{s t}^{2}\right)\left|u_{s}\right|^{\varkappa}+u_{s t}^{\varkappa, \natural} .
$$

Defining

$$
v_{t}(x):=\left|u_{t}(x)\right|^{\varkappa / 2},
$$

we have the identities:

$$
v \partial_{i} v=\frac{\varkappa}{2}\left(\partial_{i} u\right)|u|^{\varkappa-1} \quad \partial_{i} v \partial_{j} v=\frac{\varkappa^{2}}{4}\left(\partial_{i} u\right)\left(\partial_{j} u\right)|u|^{\varkappa-2} .
$$


Hence denoting by $\left(\mathbf{f}^{i}\right)$ any antiderivative of $f$, and by $v^{2, \natural}:=|u|^{\varkappa, \natural}$, it holds for every $\phi \in W^{3, \infty}$ :

$$
\begin{aligned}
& \left\langle\delta\left(v^{2}\right)_{s t}, \phi\right\rangle-\left\langle\left(B_{s t}^{1}+B_{s t}^{2}\right)\left(v_{s}^{2}\right)+v_{s t}^{2, \natural}, \phi\right\rangle \\
& =\iint_{[s, t] \times \mathbb{R}^{d}}\left[-4\left(\frac{\varkappa-1}{\varkappa}\right) a^{i j}\left(\partial_{i} v\right)\left(\partial_{j} v\right) \phi-2 a^{i j} v\left(\partial_{i} v\right)\left(\partial_{j} \phi\right)\right] \mathrm{d} x \mathrm{~d} r \\
& +\iint_{[s, t] \times \mathbb{R}^{d}}\left[-2(\varkappa-1) \mathbf{f}^{i}\left(\partial_{i} v\right) v^{1-\frac{2}{\varkappa}} \phi-\varkappa \mathbf{f}^{i} v^{2-\frac{2}{\varkappa}} \partial_{i} \phi\right] \mathrm{d} x \mathrm{~d} r=:\left\langle\delta \mathscr{D}_{s t}^{(\varkappa)}, \phi\right\rangle(.6 .11)
\end{aligned}
$$

Next, define two cylinders $Q, Q^{\prime}$ as follows: let $R, \tau>0$, and introduce

$$
\begin{gathered}
Q^{\prime}:=\{(t, x): \quad 2 \tau \leq t \leq T \text { and }|x| \leq R / 2\} \\
Q:=\{(t, x): \quad \tau \leq t \leq T \text { and }|x| \leq R\} .
\end{gathered}
$$

Since $\tau^{\prime}>0$ and $R>0$ are arbitrary, is is obviously sufficient to show the local $L^{\infty}$ estimate in $Q^{\prime}$ instead of any compact set of $(0, T] \times \mathbb{R}^{d}$.

To this end, let for each $n \geq 0$

$$
\begin{aligned}
R_{n} & :=\frac{R}{2}\left(1+2^{-n}\right) \underset{n \rightarrow \infty}{\searrow} \frac{R}{2} \\
\tau_{n} & :=\tau\left(2-2^{-n}\right) \underset{n \rightarrow \infty}{\nearrow} 2 \tau
\end{aligned}
$$

and define the cylinders $Q_{n}$ accordingly. With this definition, observe that $\tau_{0}=\tau, R_{0}=R$ and that for each $n \geq 0$

$$
Q^{\prime}=\cap_{k=0}^{\infty} Q_{k} \subset Q_{n+1} \subset Q_{n} \subset Q=Q_{0} .
$$

Now, choose any sequence of smooth test functions $\psi$. $(n ; \cdot)$ such that

$$
\psi_{t}(n ; x)=\left\{\begin{array}{l}
1 \text { for }(t, x) \in Q_{n+1} \\
0 \text { for }(t, x) \in\left([0, T] \times \mathbb{R}^{n}\right) \backslash Q_{n}
\end{array},\right.
$$

and such that

$$
\sup _{(t, x) \in[0, T] \times \mathbb{R}^{d}}\left(\left|\partial_{t} \psi_{t}(n ; x)\right|+\sum_{i=0}^{3}\left|\nabla^{i} \psi_{t}(n ; x)\right|\right) \leq C 8^{n},
$$

where the constant $C>0$ is independent of $n \geq 0$ (it is easy to see that such sequence exists).

Since $\psi(n)$ is smooth in time, thanks to the identity $\delta\left(v^{2} \psi(n)\right)_{s t}=\delta v_{s t}^{2} \psi_{s}+v_{t}^{2} \delta \psi_{s t}(n)$, we have for any $s, t \geq 0$ such that $\tau_{n} \leq s \leq t \leq T$ :

$$
\begin{aligned}
& \delta\left(\int_{\mathbb{R}^{d}} v^{2} \psi(n) \mathrm{d} x\right)_{s t}+\iint_{[s, t] \times \mathbb{R}^{d}}|\nabla v|^{2} \psi_{s}(n) \mathrm{d} x \mathrm{~d} r \\
& \lesssim \lambda \iint_{[s, t] \times \mathbb{R}^{d}}\left[\left|v_{t}\right|^{2}\left|\partial_{t} \psi(n)\right|+|v||\nabla v|\left|\nabla \psi_{s}(n)\right|+\varkappa|\mathbf{f}||\nabla v||v|^{1-\frac{2}{\varkappa}} \psi_{s}(n)\right. \\
& \left.+\varkappa|\mathbf{f}||v|^{2-\frac{2}{\varkappa}}\left|\nabla \psi_{s}(n)\right|\right] \mathrm{d} x \mathrm{~d} r \\
& \quad+\left(\left|\psi_{s}(n)\right|_{W^{1, \infty}} \omega_{B}(s, t)^{\alpha}+\left|\psi_{s}(n)\right|_{W^{2, \infty}} \omega_{B}(s, t)^{2 \alpha}\right) \int_{\mathbb{R}^{d}} v_{s}^{2} \mathrm{~d} x \\
& +\left|v_{s t}^{2, \natural}\right|_{W^{-3,1}}\left|\psi_{S}(n)\right|_{W^{3, \infty}} .
\end{aligned}
$$

Making use of the following estimates for $\varkappa \geq 2$ :

$$
v^{1-2 / \varkappa} \leq 1+v, \quad v^{2-2 / \varkappa} \leq 1+v^{2},
$$


and then letting

$$
\rho:=\frac{2 r}{r-1} \quad \text { and } \quad \sigma:=\frac{2 q}{q-1},
$$

we infer thanks to Hölder Inequality that

$$
\begin{aligned}
& \left|\delta \mathscr{D}_{s t}^{(\varkappa)}\right|_{W^{-1,1}} \lesssim\|\nabla v\|_{2,2}^{2}+\|v \nabla v\|_{1,1} \\
& +\varkappa\left(\|\mathbf{f}\|_{2 r, 2 q}\|\nabla v\|_{2,2}\|v\|_{\rho, \sigma}+\|\mathbf{f}\|_{r, q}\|v\|_{\rho, \sigma}^{2}+\|\mathbf{f}\|_{2,2}\|\nabla v\|_{2,2}+\|\mathbf{f}\|_{1,1}\right) .
\end{aligned}
$$

where for notational ease we now use the shorthand notation:

$$
\|\cdot\|_{a, b}:=\|\cdot\|_{L^{a}\left(s, t ; L^{b}\right)} \cdot
$$

Going back to (6.12) and applying Proposition 3.3 and Hölder Inequality, we obtain the inequality

$$
\begin{aligned}
& E_{Q_{n+1}}:=\sup _{\tau_{n+1}<t<T} \int_{|x|<R_{n+1}}\left|v_{t}\right|^{2} \mathrm{~d} x+\iint_{\tau_{n+1}<t<T,|x|<R_{n+1}}\left|\nabla v_{t}\right|^{2} \mathrm{~d} x \mathrm{~d} t \\
& \leq C(r, q, \lambda) 8^{n} \varkappa^{2}\left(E_{Q_{n}}+\|\mathbf{f}\|_{2,2}^{2}+\|\mathbf{f}\|_{1,1}+\left(\|\mathbf{f}\|_{2 r, 2 q}^{2}+\|\mathbf{f}\|_{r, q}\right)\left\|v \mathbf{1}_{Q_{n}}\right\|_{\rho, \sigma}^{2}\right) .
\end{aligned}
$$

where $\mathbf{1}_{Q_{n}}(x)$ is the indicator function of $Q_{n}$, and where the above constant depends on the indicated quantities but not on $\varkappa \geq 2$.

We now want to apply Lemma 6.1. To this end, observe first that thanks to (6.8), there exists $\epsilon>0$ such that

$$
\frac{1}{r}+\frac{d(1+\epsilon q)}{2 q} \leq 1
$$

For such $\epsilon>0$, is is easily seen that

$$
\frac{1}{\rho(1+\epsilon)}+\frac{d}{2(1+\epsilon) \sigma} \geq \frac{d}{4},
$$

which means in particular that the exponents

$$
\rho(1+\epsilon), \quad \sigma(1+\epsilon)
$$

still satisfy the condition (6.2).

Let $n \geq 0$. In (6.14), making the substitution $\varkappa:=\varkappa_{n}=2(1+\epsilon)^{n}$, we obtain thanks to Proposition 6.1

$$
\begin{aligned}
& \left\||u|^{(1+\epsilon)^{n}} \mathbf{1}_{Q_{n+1}}\right\|_{\rho(1+\epsilon), \sigma(1+\epsilon)} \\
& \leq C\left(E_{Q_{n+1}}\right)^{1 / 2} \\
& \leq \widetilde{C} 8^{n}(1+\epsilon)^{n}\left(1+\left(E_{Q_{n}}\right)^{1 / 2}+\left\||u|^{(1+\epsilon)^{n}} \mathbf{1}_{Q_{n}}\right\|_{\rho, \sigma}\right) .
\end{aligned}
$$

from which it follows that

$$
\left\|u \mathbf{1}_{Q_{n+1}}\right\|_{\rho(1+\epsilon)^{n}}^{\left(1+\epsilon, \sigma(1+\epsilon)^{n+1}\right.} \leq \widetilde{C} 8^{n}(1+\epsilon)^{n}\left(1+\left(E_{Q_{n}}\right)^{1 / 2}+\left\|u \mathbf{1}_{Q_{n}}\right\|_{\rho(1+\epsilon)^{n}, \sigma(1+\epsilon)^{n}}^{(1+\epsilon)^{n}}\right),
$$

where to obtain the first estimate we have used the interpolation inequality (6.5) on $|u|^{(1+\epsilon)^{n}}$. Otherwise stated, if one defines the sequence

$$
\Phi_{n}:=1+E_{Q_{n}}^{1 / 2}+\left\|u \mathbf{1}_{Q_{n}}\right\|_{\rho(1+\epsilon)^{n+1}, \sigma(1+\epsilon)^{n+1}}^{(1+\epsilon)^{n}}, \quad n \geq 0,
$$

one sees that for every $n \geq 0$ :

$$
\Phi_{n+1} \leq \gamma[8(1+\epsilon)]^{n} \Phi_{n}^{1+\epsilon}
$$


for some constant $\gamma=\gamma\left(\lambda, r, q,\|f\| \mathscr{M}, \omega_{B}, \alpha\right)>0$. Applying now (6.7), this yields for every $n \in \mathbb{N}_{0}$ :

$$
\Phi_{n} \leq \gamma^{\frac{(1+\epsilon)^{n}-1}{\epsilon}}[8(1+\epsilon)]^{\frac{(1+\epsilon)^{n}-1}{\epsilon^{2}}-\frac{n}{\epsilon}}\left\|u \mathbf{1}_{Q}\right\|_{\frac{2 r}{r-1}, \frac{2 q}{q-1}}^{(1+\epsilon)^{n}},
$$

and it follows that

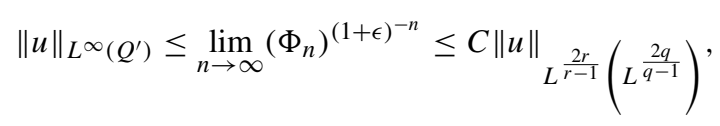

for another constant $C>0$ as above. By estimating the right hand side thanks to another application of the interpolation inequality, Proposition 6.1, we obtain the following $L^{\infty}$ bound

$$
\|u\|_{L^{\infty}\left(Q^{\prime}\right)} \leq C^{\prime}\left(\|u\|_{L^{\infty}\left(L^{2}\right)}+\|u\|_{L^{2}\left(H^{1}\right)}\right),
$$

but using the same Gronwall argument as in Section 5, this quantity is in turn bounded in terms of $\lambda, \alpha, \omega_{B},\left|u_{0}\right|_{L^{2}}$ and $\|f\|_{L^{2}\left(H^{-1}\right)}$.

Having this apriori estimate at hand, we can now proceed to the proof of Proposition 6.2.

\subsection{Proof of Proposition 6.2}

Consider an approximating sequence $\mathbf{B}(n)=S_{2}(\mathbf{B}(n))$ as in Definition 2.2. By the classical PDE theory, if we denote by $u(n)$ the corresponding weak solution (in the sense of distributions) of

$$
\begin{array}{r}
\frac{\partial u(n)}{\partial t}-A u(n)=f+\dot{B}(n) u(n) \quad \text { on }[0, T] \times \mathbb{R}^{d}, \\
u_{0}(n)=u^{0} .
\end{array}
$$

then $u(n)$ is well defined and unique in the class $L^{\infty}\left(L^{2}\right) \cap L^{2}\left(H^{1}\right)$. It is easily seen that in fact, $u(n) \in \mathcal{H}_{B}^{\alpha, 2}$ and is an $L^{2}$-energy solution of

$$
\mathrm{d} u(n)=(A u(n)+f) \mathrm{d} t+\mathrm{d} \mathbf{B}(n) u(n) .
$$

Moreover, for $f$ as in (6.1), it is known that $u(n)$ is continuous as a mapping from $[0, T] \times$ $\mathbb{R}^{d}$ to $\mathbb{R}$ (it is even $\gamma$-Hölder for some $\gamma(\lambda)>0$ [53]). For such level of regularity, it is shown by classical arguments (see for instance [46, Chapter 3]) that $v(n):=|u(n)|^{\varkappa / 2}$ satisfies the chain rule (6.9), where $\mathbf{B}$ is replaced by $\mathbf{B}(n)$. Consequently, the analysis made in the above paragraph ensures that for any compact set

$$
Q \subset \subset(0, T] \times \mathbb{R}^{d}
$$

there is a constant $C_{Q}>0$ which is independent of $n \geq 0$ such that

$$
\|u(n)\|_{L^{\infty}(Q)} \leq C_{Q},
$$

Using Banach Alaoglu Theorem, the weak-* lower-semicontinuity of the essential supremum, and also the uniqueness of the limit $u$ in $L^{\infty}\left(L^{2}\right) \cap L^{2}\left(H^{1}\right)$, we see that $u$ satisfies the same estimate. This proves the proposition.

\section{Proof of Itô Formulas}

In order to prove Theorem 2.2, we first demonstrate that the Itô Formula holds when $u$ is locally bounded and $F$ is admissible. The proof of this fact is based on a reiteration 
of the product formula obtained in Section 4, allowing to show the claimed property on polynomials of a solution. The fact that polynomials are dense in $C^{2}$ is then used together with the remainder estimates of Section 4 (it should be noted that this approach is similar to that of [58, Theorem (3.3)]). Approximating our solution by a sequence of such locally bounded elements, we will then show that the latter formula is preserved at the limit, proving the result in the general case.

\subsection{Case when $u$ is locally bounded}

Let $u$ be an $L^{2}$-energy solution of

$$
\begin{array}{r}
\mathrm{d} u=(A u+f) \mathrm{d} t+\mathrm{d} \mathbf{B} u \\
u_{0} \in L^{2},
\end{array}
$$

where $f$ belongs to $L^{2}\left(H^{-1}\right)$, and such that moreover $\|u\|_{L^{\infty}(Q)}<\infty$, for any $Q \subset \subset$ $(0, T] \times \mathbb{R}^{d}$.

Fix a compact set of the form $Q:=[\tau, T] \times K$, where $K$ is compact and $\tau>0$. If $P$ is a polynomial we infer by linearity and Corollary 4.1 that $P \circ u \in \mathcal{H}_{B}^{\alpha, 1}(Q)$ and that

$$
\mathrm{d} P(u)=P^{\prime}(u)(A u+f) \mathrm{d} t+\mathrm{d} \mathbf{B} P(u), \quad \text { on }[\tau, T] \times K,
$$

in the $L^{1}(K)$-sense.

Since $P$ is admissible, i.e., $P^{\prime}(0)=P^{\prime \prime}(0)=0$ and $\left|P^{\prime \prime}\right|_{L \infty}<\infty$, then the inequalities

$$
\begin{aligned}
& |P(z)| \leq|z|^{2}\left|P^{\prime \prime}\right|_{L^{\infty}}, \\
& \left|P^{\prime}(z)\right| \leq|z|\left|P^{\prime \prime}\right|_{L^{\infty}}, \quad \forall z \in \mathbb{R},
\end{aligned}
$$

ensure that $P \circ u$ belongs to $L^{\infty}\left(0, T ; L^{1}\left(\mathbb{R}^{d}\right)\right)$ and similarly that $\left|\nabla u \| P^{\prime}(u)\right|$ is an element of $L^{1}\left(0, T ; L^{1}\left(\mathbb{R}^{d}\right)\right)$. Hence, a direct evaluation shows that for $P$ as above, it holds

$$
\|P(u)\|_{L^{\infty}\left(L^{1}\right) \cap L^{1}\left(W^{1,1}\right)} \leq C\left(\left|P^{\prime \prime}\right|_{L^{\infty}(\mathbb{R})}\right)\|u\|_{L^{\infty}\left(L^{2}\right) \cap L^{2}\left(H^{1}\right)} .
$$

Similarly, the drift term $\mathscr{D}:=\int_{0}^{\cdot} P^{\prime}(u)(A u+f) \mathrm{d} r$ belongs to $\mathcal{V}_{1}^{1}\left(0, T ; W^{-1,1}\left(\mathbb{R}^{d}\right)\right)$ as can be seen by the estimate

$$
\begin{aligned}
& \left|\delta \mathscr{D}_{s t}\right|_{W^{-1,1}} \leq \int_{s}^{t}\left|P^{\prime}(u)(A u+f)\right|_{W^{-1,1}} \mathrm{~d} r=: \omega_{\mathscr{D}}(s, t) \\
& \leq C\left(\lambda,\|u\|_{L^{\infty}\left(L^{2}\right) \cap L^{2}\left(H^{1}\right)},\|f\|_{L^{2}\left(H^{-1}\right)},\left|P^{\prime \prime}\right|_{L^{\infty}}\right) .
\end{aligned}
$$

Hence, from Proposition 3.3, we obtain the following estimate in $\mathcal{H}_{B}^{\alpha, 1}(Q)$ :

$$
\|P(u)\|_{\mathcal{H}_{B}^{\alpha, 1}(Q)} \leq C\left(\lambda,\|u\|_{L^{\infty}\left(L^{2}\right) \cap L^{2}\left(H^{1}\right)},\|f\|_{L^{2}\left(H^{-1}\right)},\left|P^{\prime \prime}\right|_{L^{\infty}(\mathbb{R})}\right) .
$$

Denote by $\mathcal{P}_{\text {adm }}$ the set of admissible polynomials as above, equipped with the norm

$$
|P|_{C_{\text {adm }}^{2}}:=\left|P^{\prime \prime}\right|_{L^{\infty}(\mathbb{R})}
$$

The estimate (7.4) shows that we have constructed a map

$$
\begin{gathered}
\varphi_{u}: \mathcal{P}_{\mathrm{adm}} \longrightarrow \mathcal{H}_{B, \mathrm{loc}}^{\alpha, 1}\left((0, T] \times \mathbb{R}^{d}\right), \\
P \longmapsto \varphi_{u}(P):=P \circ u,
\end{gathered}
$$

which is linear and bounded. By a classical result of functional analysis, it can therefore be uniquely extended to a mapping

$$
u^{*}: C_{\mathrm{adm}}^{2} \longrightarrow \mathcal{H}_{B, \mathrm{loc}}^{\alpha, 1}\left((0, T] \times \mathbb{R}^{d}\right)
$$


which satisfies the same estimates as $\varphi_{u}$, namely (7.4) holds with $F \in C_{\text {adm }}^{2}$ instead of $P$. Considering any converging sequence $P_{n} \rightarrow F$ in $C_{\text {adm }}^{2}$, and then making use of Lemma 4.1 , it is easily checked that $\left(u^{*}(F)\right)_{t}(x)=F\left(u_{t}(x)\right)$, for every $t \in[0, T]$ and almost every $x \in \mathbb{R}^{d}$. This demonstrates in particular that $F \circ u$ is a well-defined element of $\mathcal{H}_{B, \text { loc }}^{\alpha, 1}\left((0, T] \times \mathbb{R}^{d}\right)$ and that in the $L^{1}$-sense:

$$
\mathrm{d}(F(u))=F^{\prime}(u)(A u+f) \mathrm{d} t+\mathrm{d} \mathbf{B}(F(u)) \quad \text { on }[\tau, T] \times K .
$$

Since by assumption, $u$ belongs to the class $\mathcal{H}_{B}^{\alpha, 2}\left([0, T] \times \mathbb{R}^{d}\right)$ and $F$ is admissible, neither of the terms in the right hand side of (7.4), with $P$ replaced by $F$, depend on the choice of $Q \subset \subset \mathbb{R}^{d}$. It is therefore easy exercise left to the reader that the localization (with respect to both variables) can be removed. Hence (7.6) holds in fact on $[0, T] \times \mathbb{R}^{d}$, which shows the claimed Itô formula when $u$ is locally bounded.

We can now turn to the proof of the general case.

\subsection{Proof of Theorem 2.2}

By density one can consider sequences $(f(n))$ and $\left(u_{0}(n)\right)$ such that for every $n \in \mathbb{N}_{0}, f(n)$ satisfies Assumption 6.1, and such that as $n \rightarrow \infty$ :

$$
f(n) \rightarrow f \quad \text { strongly in } L^{2}\left(H^{-1}\right) .
$$

By Proposition 6.2, the corresponding solution $u(n) \in \mathcal{H}_{B}^{\alpha, 2}$ is locally bounded away from $t=0$, and moreover, by the continuity shown in Theorem 5.1 we have

$$
u(n) \rightarrow u \text { strongly in } L^{\infty}\left(L^{2}\right) \cap L^{2}\left(H^{1}\right) .
$$

Moreover, from (7.8), there exists a subsequence (still denoted by $u(n)$ in the sequel) such that

$$
u(n) \rightarrow u \quad \text { almost everywhere on }[0, T] \times \mathbb{R}^{d} .
$$

By the intermediate result shown in the above paragraph, if $F \in C_{\text {adm }}^{2}$, we have $F(u(n)) \equiv$ $u(n)^{*}(F) \in \mathcal{H}_{B}^{\alpha, 1}$, and moreover, for every $\phi \in W^{3, \infty}$ :

$$
\begin{aligned}
& \langle\delta F(u(n)), \phi\rangle-\left\langle\left(B_{s t}^{1}+B_{s t}^{2}\right)\left[F\left(u_{s}(n)\right)\right]+F(u(n))_{s t}^{\natural}, \phi\right\rangle \\
& =-\iint_{[s, t] \times \mathbb{R}^{d}}\left[a^{i j} F^{\prime}(u(n)) \partial_{j} u(n) \partial_{i} \phi+a^{i j} F^{\prime \prime}(u(n)) \partial_{j} u(n) \partial_{i} u(n) \phi\right. \\
& \left.+\mathbf{f}^{i}(n) \partial_{i} u(n) F^{\prime \prime}(u(n)) \phi+\mathbf{f}^{i}(n) F^{\prime}(u(n)) \partial_{i} \phi+\mathbf{f}^{0}(n) F^{\prime}(u(n)) \phi\right] \mathrm{d} x \mathrm{~d} r,
\end{aligned}
$$

where $\left(\mathbf{f}^{i}(n)\right)_{i=0, \ldots, d}$, denotes any anti-derivative associated with $f(n)$.

As mentioned before, for each $n \in \mathbb{N}$, the operator norm of the extended linear map $u(n)^{*}$, which is defined in (7.5), is the same as that of $\varphi_{u}$. As a consequence, the estimate (7.4) remains true if the polynomial $P$ is replaced by $F$. In particular, there is a constant $C$ such that for any $n \in \mathbb{N}$ :

$$
\|F(u(n))\|_{\mathcal{H}_{B}^{\alpha, 1}} \leq C .
$$

By Lemma 4.1, the conclusion will follow by (7.9) and identification of the weak limits, provided one can show that

$$
\left(v(n) ; g^{0}(n), g^{i}(n)\right):=\left(F(u(n)) ; a^{i j} \partial_{i} u(n) \partial_{j} u(n), a^{i j} \partial_{j} F^{\prime}(u(n))\right), \quad n \in \mathbb{N}_{0},
$$

is uniformly integrable. 
But using the pointwise estimates $|v(n)| \lesssim u(n)^{2},\left|g^{0}(n)\right| \lesssim|\nabla u(n)|^{2}$ and $\left|g^{i}(n)\right| \lesssim$ $|\nabla u(n)|^{2}+|u(n)|^{2}$, this property is an obvious consequence of the strong convergence (7.8). This finishes the proof of Theorem 2.2-(i). The proof of the second item is similar and therefore omitted.

\subsection{The $L^{p}$-norm of $L^{p}$ solutions: proof of Corollary 2.1}

For $R>0$ we define an admissible truncation $F_{R}$ of $|\cdot|^{p}$ as follows. Let $\theta \in C_{c}^{\infty}$, supported in $[0,2)$ such that $\theta=1$ on $[0,1]$ while $0 \leq \theta \leq 1$. Define

$$
F_{R}(z):=\int_{0}^{|z|} \mathrm{d} y \int_{0}^{y} \theta\left(\frac{|\tau|}{R}\right) p(p-1)|\tau|^{p-2} \mathrm{~d} \tau, \quad z \in \mathbb{R} .
$$

Clearly, $\left|F_{R}^{\prime \prime}\right|_{L^{\infty}}<\infty$, and $F_{R}(0)=F_{R}^{\prime}(0)=0$, so $F_{R}$ is admissible. Moreover, as $R \rightarrow$ $\infty, F_{R} \nearrow|\cdot|^{p}$ almost everywhere and locally uniformly.

We have by Theorem 2.2:

$$
\begin{aligned}
&\left\langle\delta F_{R}(u)_{s t}-\left(B_{s t}^{1}+B_{s t}^{2}\right)\left[F_{R}(u)\right]-F_{R}(u)_{s t}^{\natural}, \phi\right\rangle \\
&=-\iint_{[s, t] \times \mathbb{R}^{d}}\left[a^{i j} F_{R}^{\prime}(u) \partial_{j} u \partial_{i} \phi+a^{i j} F_{R}^{\prime \prime}(u) \partial_{j} u \partial_{i} u \phi\right. \\
&\left.+\mathbf{f}^{i} \partial_{i} u F_{R}^{\prime \prime}(u) \phi+\mathbf{f}^{i} F_{R}^{\prime}(u) \partial_{i} \phi\right] \mathrm{d} x \mathrm{~d} r \\
& \lesssim_{\lambda, p, \theta}|\phi|_{W^{1, \infty}}\left(\iint_{[s, t] \times \mathbb{R}^{d}}|u|^{p-1}|\nabla u|+|u|^{p-2}|\nabla u|^{2}+|\mathbf{f}\|\nabla u\| u|^{p-2}+|\mathbf{f}||u|^{p-1}\right) \\
& \lesssim_{\lambda, p, \theta}\|u\|_{L^{p}\left(L^{p}\right)}^{p-1}\left(\|\nabla u\|_{L^{p}\left(L^{p}\right)}+\|f\|_{L^{p}\left(W^{-1, p}\right)}\right) \\
& \quad+\|u\|_{L^{p}\left(L^{p}\right)}^{p-2}\left(\|\nabla u\|_{L^{p}\left(L^{p}\right)}+\|\nabla u\|_{L^{p}\left(L^{p}\right)}^{2}\|f\|_{L^{p}\left(W^{-1, p}\right)}\right) .
\end{aligned}
$$

The above drift term is therefore uniformly bounded in $R>0$, and so is $\left\|F_{R}(u)\right\|_{\mathcal{H}_{B}^{\alpha, 1}}$ by Proposition 3.3.

By Lemma 4.1, this implies that one can take limits as $R \rightarrow \infty$, in the above weak formulation. But this means that (2.33) holds, which finishes the proof.

\subsection{The $L^{p}$-norm in the general case: proof of Theorem 2.4}

Uniqueness is easy and therefore we only sketch the proof. If $u^{1}$ and $u^{2}$ are two such solutions, then $v:=u^{1}-u^{2}$ is also a solution of the same equation with 0 instead of $f$. Using the Itô formula on $|v|^{p}$, and testing against $\phi=1$, we find thanks to Proposition 3.3 that the $L^{\infty}\left(s, t ; L^{1}\right)$-norm of $v$ satisfies an incremental inequality of the form (3.7) with $\varphi(s, t)=0$. The conclusion then follows by the rough Gronwall argument, Lemma 3.1, and the fact that $v_{0}=0$.

To show existence, we first adapt the compactness argument used in Section 5 for the $L^{2}$-theory.

Step 1: compactness argument Let us first consider the case when $B=X \cdot \nabla+X^{0} \in$ $C^{\infty}\left(0, T ; \mathbb{D}_{1}\right)$, and let $u$ be the unique distributional solution of

$$
\begin{aligned}
\partial_{t} u-A u=f+\left(\dot{X} \cdot \nabla+\dot{X}^{0}\right) u & \text { on }(0, T] \times \mathbb{R}^{d}, \\
& u_{0}:=u^{0} \in L^{p} .
\end{aligned}
$$


From the classical PDE theory and our definition of the spaces $\mathcal{H}_{B}^{\alpha, p}$ it is straighforward to check that $u \in \mathcal{H}_{B}^{\alpha, 2}$. Moreover, it is standard that in the distributional sense

$$
\partial_{t}\left(|u|^{p}\right)=p u|u|^{p-2}\left(A u+\partial_{i} \mathbf{f}^{i}+\mathbf{f}^{0}\right)+\dot{X} \cdot \nabla\left(|u|^{p}\right)+p \dot{X}^{0}|u|^{p}
$$

and, by the consistence of rough integration with Lebesgue/Stieljes integration, it holds in that case

$$
\mathrm{d}|u|^{p}-p u|u|^{p-2}(A u+f) \mathrm{d} t=\mathrm{d} \mathbf{B}^{(p)}|u|^{p},
$$

in the sense of Definition 2.4 in $L^{1}$, and where $\mathbf{B}^{(p)}:=S_{2}\left(X \cdot \nabla+p X^{0}\right)$. Let $\mathbf{f}^{i} \in$ $L^{1}\left(L^{p}\right) \cap L^{2}\left(L^{2}\right), i=0, \ldots, d$ be any antiderivative of $f$. Integrating, we have using Hölder Inequality

$$
\begin{aligned}
\delta\left(|u|_{L^{p}}^{p}\right)_{s t} & +\iint_{[s, t] \times \mathbb{R}^{d}}|u|^{p-2}|\nabla u|^{2} \mathrm{~d} x \mathrm{~d} t \\
\lesssim \lambda, p & \delta\left(|u|_{L^{p}}^{p}\right)_{s t}+\iint_{[s, t] \times \mathbb{R}^{d}} p(p-1) a^{i j}|u|^{p-2} \partial_{i} u \partial_{j} u \mathrm{~d} x \mathrm{~d} t \\
= & \iint_{[s, t] \times \mathbb{R}^{d}}\left[p u|u|^{p-2} \mathbf{f}^{0}-p(p-1)|u|^{p-2} \partial_{i} u \mathbf{f}^{i}\right] \mathrm{d} x \mathrm{~d} t \\
& +\int_{\mathbb{R}^{d}}\left|u_{s}\right|^{p}\left(B_{s t}^{(p), 1, *}+B_{s t}^{(p), 2, *}\right) 1 \mathrm{~d} x+\left\langle\left|u_{s t}\right|^{p, \natural}, 1\right\rangle \\
\lesssim \lambda, p & \|u\|_{\infty, p}^{p-1}\left\|\mathbf{f}^{0}\right\|_{1, p}+\left\||u|^{p-2}|\nabla u|^{2}\right\|_{1,1}^{1 / 2}\|u\|_{\infty^{2}, p}^{\frac{p-2}{2}}\left\|\mathbf{f}^{i}\right\|_{2, p} \\
& \quad+\|u\|_{\infty, p}^{p}\left(\omega_{B}(s, t)^{\alpha}+\omega_{B}(s, t)^{2 \alpha}\right)+\llbracket|u|^{p, \natural \rrbracket} \rrbracket_{-3}^{[3 \alpha]}(s, t)
\end{aligned}
$$

where we recall the shorthand notation $\|\cdot\|_{a, b}:=\|\cdot\|_{L^{a}\left(s, t ; L^{b}\right)}$. But thanks to the remainder estimates, Proposition 3.3, we find for $|t-s| \leq L\left(\rho_{\alpha}(\mathbf{B})\right)$ small enough:

$$
\begin{aligned}
& \delta\left(|u|_{L^{p}}^{p}\right)_{s t}+\iint_{[s, t] \times \mathbb{R}^{d}}|u|^{p-2}|\nabla u|^{2} \mathrm{~d} x \mathrm{~d} t \\
& \lesssim_{\lambda, p}\left(\|u\|_{\infty, p}^{p-1}\left\|\mathbf{f}^{0}\right\|_{p, p}(t-s)^{\frac{p-1}{p}}+\left\||u|^{p-2}|\nabla u|^{2}\right\|_{1,1}^{1 / 2}\|u\|_{\infty, p}^{\frac{p-2}{2}}\left\|\mathbf{f}^{i}\right\|_{2, p}\right)\left(1+\omega_{B}(s, t)^{\alpha}\right) \\
& \quad \omega_{B}(s, t)^{\alpha}\left(\|u\|_{\infty, p}^{p-1}\left\|\mathbf{f}^{i}\right\|_{p, p}(t-s)^{\frac{p-1}{p}}+\left\||u|^{p-2}|\nabla u|^{2}\right\|_{1,1}^{1 / 2}\|u\|_{\infty, p}^{p / 2}(t-s)^{1 / 2}\right) \\
& +\|u\|_{\infty, p}^{p}\left(\omega_{B}(s, t)^{\alpha}+\omega_{B}(s, t)^{2 \alpha}+\omega_{B}(s, t)^{\alpha}\right)
\end{aligned}
$$

Using Young Inequality, taking $L\left(\rho_{\alpha}(\mathbf{B}), \lambda\right)$ smaller if necessary and then absorbing to the left, we end up with the inequality

$\delta\left(|u|_{L^{p}}^{p}\right)_{s t}+\iint_{[s, t] \times \mathbb{R}^{d}}|u|^{p-2}|\nabla u|^{2} \mathrm{~d} x \mathrm{~d} t \lesssim \lambda, p\|u\|_{\infty, p}^{p}\left[\omega_{B}(s, t)^{\alpha}+(t-s)\right]+\left\|\mathbf{f}^{0}, \mathbf{f}^{i}\right\|_{p, p}^{p}$

By the rough Gronwall Lemma, Lemma 3.1, we obtain the estimate on

$$
\|u\|_{L^{\infty}\left(L^{p}\right)}^{p}+\iint_{[0, T] \times \mathbb{R}^{d}}|u|^{p-2}|\nabla u|^{2} \mathrm{~d} x \mathrm{~d} t \leq C\left(\lambda, p, \rho_{\alpha}(\mathbf{B}),\|f\|_{L^{p}\left(W^{-1, p}\right)}\right) .
$$

Now, consider a sequence of canonical lifts $\mathbf{B}(n)=S_{2}\left(X(n) \cdot \nabla+X^{0}(n)\right)$ such that $X(n)$ is smooth in time, $\mathbf{B}(n) \rightarrow \mathbf{B}$, and define the differential rough driver $\mathbf{B}^{(p)}(n)$ correspondingly. Note that for each $n \geq 0$, the map $v(n):=|u(n)|^{p}$ belongs to $\mathcal{H}_{B^{(p)}(n)}^{\alpha, 1}$, since the smoothness of $X(n)$ in time makes trivial the statement about the remainder

$$
R_{s t}^{v(n)}=\delta v_{s t}(n)-B_{s t}^{(p), 1}(n) v_{s}(n),
$$


in the definition of the controlled path space $\mathcal{D}_{B(n)}^{\alpha, 1}$. Thanks to the convergence of $\mathbf{B}(n)$, it is immediately checked that $\rho_{\alpha}\left(\mathbf{B}^{(p)}(n), \mathbf{B}^{(p)}\right) \rightarrow 0$ (the $\rho_{\alpha}$-convergence sense is equivalent to the convergence of the coefficients, see Appendix A.1).

Moreover, thanks to the identities (6.10) and the remainder estimates (Proposition 3.3), the estimate (7.15) implies the following uniform estimate on $v(n)=|u(n)|^{p / 2}$

$$
\left\||u(n)|^{p / 2}\right\|_{\mathcal{H}_{B(p / 2)(n)}^{\alpha, 2}} \leq C\left(\lambda, p,\|f\|_{L^{p}\left(W^{-1, p}\right)}\right) .
$$

Applying Lemma 4.1, one infers the existence of $v \in \mathcal{H}_{B^{(p / 2)}}^{\alpha, 2}$ such that $v(n) \rightarrow v$ weakly-* in $L^{\infty}\left(L^{2}\right) \cap L^{2}\left(H^{1}\right)$. Interpolating the $L^{2}\left(H^{1}\right)$-estimate with the $\mathcal{V}^{\alpha}\left(H^{-1}\right)$ estimate, it is easily seen that the convergence of $v(n)$ holds strongly in $L^{2}\left(L_{\text {loc }}^{2}\right)$ and thus, upon taking a subsequence we can assume that

$$
\begin{aligned}
|u(n)|^{p / 2} & \rightarrow|u|^{p / 2}, \quad \text { in } L^{2}\left(0, T ; L_{\text {loc }}^{2}\right) \text { strong, and } \\
u(n) & \rightarrow u \text { almost everywhere in }[0, T] \times \mathbb{R}^{d} .
\end{aligned}
$$

Using again the remainder estimates, Proposition 3.3, it follows from the equation on $|u(n)|^{p}$ that

$$
\left\||u(n)|^{p}\right\|_{\mathcal{D}_{B(n)}^{\alpha, 1}} \leq C .
$$

Therefore, by the same compactness argument as in the proof of Lemma 4.1, there exists $w$ and $g^{i}, i=0 \ldots, d$ in $\left(L^{\infty}\right)^{*}$ so that for any $\Phi \in L^{\infty}\left([0, T] \times \mathbb{R}^{d}\right)$,

$$
\begin{aligned}
& \iint_{[0, T] \times \mathbb{R}^{d}}|u(n)|^{p} \Phi \mathrm{d} x \mathrm{~d} t \rightarrow\langle w, \Phi\rangle_{\left(L^{\infty}\right)^{*}, L^{\infty}} \\
& p(p-1) \iint_{[0, T] \times \mathbb{R}^{d}} a^{i j}|u(n)|^{p-2} \partial_{i} u(n) \partial_{j} u(n) \Phi \mathrm{d} x \mathrm{~d} t \rightarrow\left\langle g^{0}, \Phi\right\rangle_{\left(L^{\infty}\right)^{*}, L^{\infty}} \\
& p \iint_{[0, T] \times \mathbb{R}^{d}} u(n)|u(n)|^{p-2} \partial_{j} u(n) \Phi \mathrm{d} x \mathrm{~d} t \rightarrow\left\langle g^{i}, \Phi\right\rangle_{\left(L^{\infty}\right)^{*}, L^{\infty}, \quad i=1, \ldots, d .}
\end{aligned}
$$

It remains to show that the above limits are the expected ones (thereby proving that the above convergences hold in $L^{1}$-weak).

Identification of the limits and conclusion Using the strong convergence of $v(n)=$ $|u(n)|^{p / 2}$, we also find

$$
\begin{aligned}
\iint_{[0, T] \times \mathbb{R}^{d}}|u(n)|^{p} \Phi \mathrm{d} x \mathrm{~d} t= & \iint_{[0, T] \times \mathbb{R}^{d}}|u(n)|^{p / 2}\left(|u(n)|^{p / 2} \Phi\right) \mathrm{d} x \mathrm{~d} t \\
& \rightarrow \iint_{[0, T] \times \mathbb{R}^{d}}|u|^{p} \Phi \mathrm{d} t \mathrm{~d} x,
\end{aligned}
$$

and therefore we see that $w=|u|^{p}$. To conclude, it remains to show that

$$
\begin{aligned}
& g^{0}=a^{i j}|u|^{p-2} \partial_{i} u \partial_{j} u \\
& g^{j}=u|u|^{p-2} \partial_{j} u .
\end{aligned}
$$

We content ourselves to show the first assertion since the other one is similar.

In order to prove (7.19), observe first that since $p \geq 4$, it is also larger than 2 and thus the sequences $\left\{u(n), n \in \mathbb{N}_{0}\right\}$ and $\left\{u^{2}(n), n \in \mathbb{N}_{0}\right\}$ are also uniformly bounded in the $\mathcal{H}_{B(n) \text {,loc }}^{\alpha, 2}$ (respectively $\mathcal{H}_{B^{(2)}(n)}^{\alpha, 1}$ )-sense. The Banach Alaoglu Theorem implies the existence of $\mu \in\left(L^{\infty}\right)^{*}$ so that $a^{i j} \partial_{i} u(n) \partial_{j} u(n) \rightarrow \mu$ weakly-*. On the other hand $\nabla u(n) \rightarrow \nabla u$ in $L_{\mathrm{w}}^{2}$, and thus applying the local product formula of $u$ with itself, we find that necessarily

$$
\mu=a^{i j} \partial_{i} u \partial_{j} u \text {. }
$$


But since $p \geq 4$, replacing $p$ by $p-2$ in the previous step, we see that there exists $h^{0}$ in $\left(L^{\infty}\right)^{*}$ so that $(7.17)$ holds with $\left(p-2, h^{0}\right)$ instead of $\left(p, g^{0}\right)$, and it is easily seen that

$$
p(p-1) u^{2} h^{0}=(p-2)(p-3) g^{0} .
$$

Applying the product formula, Proposition 4.1, to $|u|^{p-2}$ with $u^{2}$, we see thanks to (7.21) that $g^{0}=u^{2} h^{0}+2 \mu|u|^{p-2}=\frac{(p-2)(p-3)}{p(p-1)} g^{0}+2 a^{i j} \partial_{i} u \partial_{j} u|u|^{p-2}$, which after simplification provides the relation (7.19).

Hence the chain rule (7.13) remains true for $u$ which we recall is the unique solution in the class described by the hypotheses of the theorem. This finishes the proof.

\section{Proof of Theorem 2.5}

We start with the following elementary observation. For a domain $D \subset \mathbb{R}^{d}$ with smooth boundary, elements of $W_{0}^{k, p}(D)$ for $0 \leq k \leq 3$ and $p \in[1, \infty]$ are naturally identified in $W^{k, p}\left(\mathbb{R}^{d}\right)$ through the embedding map

$$
\iota_{D}: W_{0}^{3, p}(D) \hookrightarrow W^{3, p}\left(\mathbb{R}^{d}\right),
$$

where for any $\phi$ in $W_{0}^{3, p}(D)$, we define

$$
\iota_{D} \phi(x):=\left\{\begin{array}{l}
\phi(x) \text { if } x \in D \\
0 \text { if } x \notin D .
\end{array}\right.
$$

This operation is of course linear and continuous. In particular, by duality, for every distribution $g \in W^{-3, p^{\prime}}\left(\mathbb{R}^{d}\right)$, the restriction $\left.g\right|_{D} \equiv \iota_{D}^{*} g$ to a smooth domain $D$ is well defined.

\subsection{Proof of the solvability}

Identify the test functions $W_{0}^{k, p}(D)$ as elements of $W^{k, p}\left(\mathbb{R}^{d}\right)$ as in the above discussion, and then define

$$
\tilde{\sigma}:=\iota_{D}(\sigma), \quad \tilde{\mathbf{B}}:=\left(\tilde{B}^{1}, \tilde{B}^{2}\right):=\left(Z^{1} \tilde{\sigma} \cdot \nabla, Z^{2}(\tilde{\sigma} \cdot \nabla)^{2}\right) .
$$

Moreover, let $\tilde{u}_{0}:=\iota_{D}\left(u_{0}\right)$. Concerning the elliptic part, we define

$$
\tilde{a}^{i j}(t, x):=\left\{\begin{array}{l}
a^{i j}(t, x) \text { if }(t, x) \in[0, T] \times D \\
\mathbf{1}_{i=j} \text { otherwise, }
\end{array}\right.
$$

and we let $\tilde{A}:=\partial_{i}\left(\tilde{a}^{i j} \partial_{j} \cdot\right)$. With these definitions, $\tilde{A}, \tilde{\mathbf{B}}$, fulfill the hypotheses of Theorem 5.1 so that there exists a unique $L^{2}$-energy solution $u \in \mathcal{H}_{B}^{\alpha, 2}\left([0, T] \times \mathbb{R}^{d}\right)$ to

$$
\mathrm{d} u=\tilde{A} u \mathrm{~d} t+\mathrm{d} \tilde{\mathbf{B}} u, \quad \text { on } \quad[0, T] \times \mathbb{R}^{d} .
$$

The restriction $v:=\left.u\right|_{[0, T] \times D}$ is the natural candidate to solve the Dirichlet problem (2.35). In order to check that this is indeed the case, let us remark that $w:=\left.u\right|_{[0, T] \times\left(\mathbb{R}^{d} \backslash D\right)}$ is a classical solution to

$$
\partial_{t} w=\Delta w \quad \text { on }[0, T] \times\left(\mathbb{R}^{d} \backslash D\right), \quad w_{0}=0,
$$

and hence $w=0$. This shows that $u$ is supported in $[0, T] \times D$. Since on the other hand $u$ belongs to $L^{2}\left(H^{1}\left(\mathbb{R}^{d}\right)\right)$, this implies that its trace onto $[0, T] \times \partial D$ is well defined, so that $v \in L^{2}\left(H_{0}^{1}(D)\right)$. This shows that $v$ solves the Dirichlet problem (2.35). 


\subsection{Proof of the maximum principle}

The proof uses the so-called Stampacchia truncatures approach. We first assume that

$$
a \in L^{1}\left(0, T ; W_{0}^{1, \infty}(D)\right) .
$$

Namely, let us fix a map $G \in C^{1}(\mathbb{R})$ such that the following properties are satisfied:

$$
\left[\begin{array}{l}
\left|G^{\prime}\right|_{L^{\infty}(\mathbb{R})}<\infty \\
G \text { is increasing on }(0, \infty) \\
G(x)=0 \text { whenever } x \leq 0
\end{array}\right.
$$

Let $F \in C^{2}(\mathbb{R})$ be defined by

$$
F(x):=\int_{0}^{x-M} G(y) \mathrm{d} y, \quad x \in \mathbb{R},
$$

where we denote by

$$
M=\max \left(0, \operatorname{ess} \sup _{D} u_{0}\right)<\infty .
$$

By Theorem 2.2 applied to $F$ (note that $u$ has compact support) the following equation holds:

$$
\left\langle\delta F(u)_{s t}, \phi\right\rangle=\int_{s}^{t}\left\langle G\left(u_{r}-M\right) A u_{r}, \phi\right\rangle \mathrm{d} r+\left\langle\left(B^{1}+B^{2}\right)_{s t} F\left(u_{s}\right), \phi\right\rangle+\left\langle F_{s t}^{\natural}, \phi\right\rangle,
$$

for some remainder $F^{\natural} \in \mathcal{V}^{1+}\left(0, T ; W^{-3,1}\right)$. Next, we arrange the drift term as follows:

$$
\begin{aligned}
\langle G(u-M) A u, \phi\rangle+\left\langle a^{i j} G^{\prime}(u-M) \partial_{i} u \partial_{j} u, \phi\right\rangle & =\left\langle-a^{i j} G(u-M) \partial_{j} u, \partial_{i} \phi\right\rangle \\
& =\left\langle F(u), \partial_{j}\left(a^{i j} \partial_{i} \phi\right)\right\rangle .
\end{aligned}
$$

Hence, denoting by $\mathscr{D}:=\int_{0}^{\cdot} G\left(u_{r}-M\right) A_{r} u_{r} \mathrm{~d} r$, we have for each $(s, t) \in \Delta$ :

$$
\left|\delta \mathscr{D}_{s t}\right|_{W^{-2,1}} \leq \lambda^{-1} \iint_{[s, t] \times D} G^{\prime}(u-M)|\nabla u|^{2} \mathrm{~d} x \mathrm{~d} r+\|a\|_{L^{1}\left(s, t ; W^{1, \infty}\right)}\|F(u)\|_{L^{\infty}\left(s, t ; L^{1}\right)} .
$$

Therefore, testing the equation against $\phi=1$ and then using Assumption 2.1 gives

$$
\begin{aligned}
& \delta\left(|F(u)|_{L^{1}}\right)_{s t}+\iint_{[s, t] \times D} G^{\prime}(u-M)|\nabla u|^{2} \mathrm{~d} x \mathrm{~d} r \\
& \lesssim_{\lambda} \lambda^{-1}\|F(u)\|_{L^{\infty}\left(s, t ; L^{1}\right)} \omega_{B}(s, t)^{\alpha}+\|F(u)\|_{L^{\infty}\left(s, t ; L^{1}\right)}\|a\|_{L^{1}\left(s, t ; W^{1, \infty}\right)},
\end{aligned}
$$

for any $(s, t)$ such that $\omega_{B}(s, t) \leq L(\lambda)$. Applying Lemma 3.1, we obtain that

$$
\|F(u)\|_{L^{\infty}\left(L^{1}\right)} \leq C\left(\lambda,\|a\|_{L^{1}\left(W^{1, \infty}\right)}, \omega_{B}, \alpha\right)\left|F\left(u_{0}\right)\right|_{L^{1}} \equiv 0,
$$

from which we conclude that $u \leq M$ a.e. The proof of the estimate below is similar, hence omitted. This proves the desired inequality, when (8.2) holds.

For general coefficients $a^{i j}$, we consider an approximating sequence $a^{i j}(n), n \in \mathbb{N}$, which converges almost everywhere and in $L^{1}$ to $a^{i j}$, and such that for each $n$, Assumption 2.1 is satisfied (with a uniform $\lambda$ ) and (8.2) holds. By Lemma 4.1, we can assume without loss of generality that the corresponding solution $u(n)$ converges almost everywhere to that associated with $a^{i j}$. Taking the limit in (2.38) then proves the result. This finishes the proof of Theorem 2.5.

Acknowledgements Open Access funding provided by Projekt DEAL. The authors would like to thank the anonymous referee who significantly helped to improve the quality of this manuscript. 
Open Access This article is licensed under a Creative Commons Attribution 4.0 International License, which permits use, sharing, adaptation, distribution and reproduction in any medium or format, as long as you give appropriate credit to the original author(s) and the source, provide a link to the Creative Commons licence, and indicate if changes were made. The images or other third party material in this article are included in the article's Creative Commons licence, unless indicated otherwise in a credit line to the material. If material is not included in the article's Creative Commons licence and your intended use is not permitted by statutory regulation or exceeds the permitted use, you will need to obtain permission directly from the copyright holder. To view a copy of this licence, visit http://creativecommonshorg/licenses/by/4.0/.

\section{Appendix A: some technical proofs}

\section{A.1 Proof of Lemma 2.1}

It is well-known that a multiplication operator $M_{f}$ of the form $M_{f} h:=x \mapsto f(x) h(x)$ for $h \in L^{2}$, is bounded if and only if $|f|_{L^{\infty}}<\infty$, and that the map $f \in L^{\infty} \mapsto M_{f} \in$ $\mathscr{L}\left(L^{2}, L^{2}\right)$ is an isometry (see for instance [57]). By an immediate generalization, for $i=$ 1,2 , we see that the couple $\left(j_{1}, j_{2}\right)$ defined as

$$
\begin{array}{ll}
j_{1}:\left(W^{3, \infty}\right)^{d} \times W^{2, \infty} \rightarrow \mathbb{D}_{1} & (X, Y) \mapsto X^{i} \partial_{i}+Y, \\
j_{2}:\left(W^{3, \infty}\right)^{d \times d} \times\left(W^{2, \infty}\right)^{d} \times W^{1, \infty} \rightarrow \mathbb{D}_{2} & (\mathbb{X}, \mathbb{Y}, \mathbb{Z}) \mapsto \mathbb{X}^{i j} \partial_{i j}+\mathbb{Y}^{i} \partial_{i}+\mathbb{Z},(\text { A. } 1)
\end{array}
$$

is a continuous isomorphism, where $\mathbb{D}_{i}, i=1,2$, are equipped with the operator-norm topologies as in Definition 2.1 .

Let $t \mapsto B_{t}=X_{t} \cdot \nabla+X_{t}^{0}$ be in $C^{1}\left(0, T ; \mathbb{D}_{1}\right)$ and, as in (2.11), define the canonical lift

$$
\left(B^{1}, B^{2}\right):=S_{2}(B) \text {. }
$$

By definition of $B_{s t}^{2}$, we have for $0 \leq s \leq t \leq T$ :

$$
\begin{aligned}
B_{s t}^{2} & :=\int_{s}^{t} \mathrm{~d} B_{r} \circ \delta B_{s r} \\
& =\int_{s}^{t}\left(\mathrm{~d} X_{r}^{i} \partial_{i}+\mathrm{d} X_{r}^{0}\right) \circ\left(X_{s r}^{j} \partial_{j}+X_{s r}^{0}\right) \\
& =\mathbb{X}_{s t}^{i j} \partial_{i j}+\left(\mathbb{L}_{s t}^{i}+2 \mathbb{S}_{s t}^{0 i}\right) \partial_{i}+\mathbb{L}_{s t}^{0}+\mathbb{S}_{s t}^{00},
\end{aligned}
$$

where we recall the notation $X_{s t}:=X_{t}-X_{s}$, and where we introduce

$$
\left[\begin{array}{l}
\mathbb{X}_{s t}^{i j}=\int_{s}^{t} X_{s r}^{i} \mathrm{~d} X_{r}^{j} \\
\mathbb{L}_{s t}^{i}=\int_{s}^{t} \mathrm{~d} X_{r}^{\mu} \partial_{\mu} X_{s r}^{i}, \\
\mathbb{S}_{s t}^{i j}=\operatorname{sym} \mathbb{X}_{s t}^{i j}:=\frac{1}{2}\left(\int_{s}^{t} X_{s r}^{i} \mathrm{~d} X_{r}^{j}+\int_{s}^{t} X_{s r}^{j} \mathrm{~d} X_{r}^{i}\right), \quad \text { for all } 0 \leq i, j \leq d .
\end{array}\right.
$$

The above integrals are understood in the sense of Bochner, in $W^{3, \infty}, W^{2, \infty}, W^{1, \infty}$. As seen through immediate algebraic computations, the generalized Chen's relations (2.17) hold in this case, since

$$
\begin{aligned}
\delta \mathbb{X}_{s \theta t} & =\mathbb{L}_{s t}^{i}-\mathbb{L}_{s \theta}^{i}-\mathbb{L}_{\theta t}^{i} \\
& =\left(\int_{s}^{t}-\int_{s}^{\theta}\right)\left(\mathrm{d} X_{r}^{\mu} \partial_{\mu} X_{s r}^{i}\right) \mathrm{d} r-\int_{\theta}^{t}\left(\mathrm{~d} X_{r}^{\mu} \partial_{\mu} X_{\theta r}^{\mu}\right) \mathrm{d} r \\
& =X_{\theta t}^{\mu} \partial_{\mu} X_{s \theta}^{i} .
\end{aligned}
$$


Next, for almost every $x \in \mathbb{R}^{d}$, an integration by parts in the time variable yields the identity

$$
\mathbb{S}_{s t}^{i j}(x)=\frac{1}{2} X_{s t}^{i}(x) X_{s t}^{j}(x), \quad i=0, \ldots d .
$$

Denoting by $\mathbb{A}_{s t}^{i j}:=\mathbb{X}_{s t}^{i j}-\mathbb{S}_{s t}^{i j}$, we further observe that Schwarz Theorem implies

$$
\mathbb{X}_{s t}^{i j} \partial_{i j}=\mathbb{S}_{s t}^{i j} \partial_{i j}+\mathbb{A}_{s t}^{i j} \partial_{i j}=\mathbb{S}_{s t}^{i j} \partial_{i j},
$$

since $\mathbb{A}_{s t}$ is antisymmetric. Hence, only the symmetric part of $\mathbb{X}$ contributes to the second order part of $B_{s t}^{2}$ in (A.2). This yields the desired expression, namely

$$
B_{s t}^{2}=\frac{1}{2} X_{s t}^{i} X_{s t}^{j} \partial_{i j}+\left(\mathbb{L}_{s t}^{i}+X_{s t}^{0} X_{s t}^{i}\right) \partial_{i}+\mathbb{L}_{s t}^{0}+\frac{1}{2}\left(X_{s t}^{0}\right)^{2} .
$$

To show (2.1), note that

$$
\begin{aligned}
B_{s t}^{1} \circ B_{s t}^{1} & =\left(X_{s t}^{i} \partial_{i}+X_{s t}^{0}\right) \circ\left(X_{s t}^{j} \partial_{j}+X_{s t}^{0}\right) \\
& =X_{s t}^{i} X_{s t}^{j} \partial_{i j}+\left(X_{s t}^{j} \partial_{j} X_{s t}^{i}+2 X_{s t}^{0} X_{s t}^{i}\right) \partial_{i}+X_{s t}^{j} \partial_{j} X_{s t}^{0}+\left(X_{s t}^{0}\right)^{2}
\end{aligned}
$$

This yields, by definition of $[\mathbf{B}]$ :

$$
\begin{aligned}
{[\mathbf{B}]_{s t} } & \equiv B_{s t}^{2}-\frac{1}{2} B_{s t}^{1} \circ B_{s t}^{1} \\
& =\left(\mathbb{L}_{s t}^{i}-\frac{1}{2} X_{s t}^{j} \partial_{j} X_{s t}^{i}\right) \partial_{i}+\mathbb{L}_{s t}^{0}-X_{s t}^{j} \partial_{j} X_{s t}^{0}
\end{aligned}
$$

which is the claimed equality.

Now, pick any geometric differential rough driver $\mathbf{B}$, and let $\mathbf{B}(n) \in C^{1}\left(0, T ; \mathbb{D}_{1}\right), n \in$ $\mathbb{N}_{0}$, be such that $\mathbf{B}(n) \equiv S_{2}(B(n)) \rightarrow_{\rho_{\alpha}}$ B. Making use of the isomorphisms $\left(j_{1}, j_{2}\right)$ we see that the coefficients

$$
(X(n), Y(n) ; \mathbb{X}(n), \mathbb{Y}(n), \mathbb{Z}(n)) \equiv\left(j_{1}^{-1} B^{1}(n) ; j_{2}^{-1} B^{2}(n)\right)
$$

converge to $\left(j_{1}^{-1} B^{1} ; j_{2}^{-1} B^{2}\right)$, in the space

$$
\left(\left(W^{3, \infty}\right)^{d} \times W^{2, \infty}\right) \times\left(\left(W^{3, \infty}\right)^{d \times d} \times\left(W^{2, \infty}\right)^{d} \times W^{1, \infty}\right) .
$$

In particular, one can take the limits in the identities (A.4), (A.6), (A.7), proving the corresponding relations for the limit $\mathbf{B}$.

\section{A.2 Renormalization property for geometric differential rough drivers}

In what follows, we fix $D \subset U \subset \mathbb{R}^{d}$ as in Section 4 and, recalling Notation 4.15, we will further denote by $\Omega:=\Omega^{D}$ while $\Omega_{\epsilon}:=\Omega_{\epsilon}^{D}$.

Given $\Phi(\cdot, \cdot)$, we have for $(x, y) \in \Omega$, by definition of $T_{\epsilon}$ :

$$
T_{\epsilon} \Phi(x, y):=\frac{1}{(2 \epsilon)^{d}} \Phi\left(x_{+}+\frac{x_{-}}{\epsilon}, x_{+}-\frac{x_{-}}{\epsilon}\right),
$$

where we introduce the new coordinates

$$
x_{+}:=\frac{x+y}{2}, \quad, x_{-}:=\frac{x-y}{2} .
$$

Note that the Jacobian determinant of the map $\chi: \Omega \rightarrow \mathbb{R}^{d} \times B_{1},(x, y) \mapsto\left(x_{+}, x_{-}\right)$is equal to $2^{-d}$ (in fact $\sqrt{2} \chi$ is a rotation). By a common abuse of notation, we will denote by $\nabla_{ \pm}$the gradient with respect to the new coodinates $x_{+}(x, y)$ and $x_{-}(x, y)$. Formally, we have the relation $\nabla_{ \pm}=\nabla_{x} \pm \nabla_{y}$. 
The proof of Theorem 4.1 is based on the following result, whose proof is implicitly contained in [19], and therefore omitted.

Lemma A.1 Let $V=\sigma^{i}(\cdot) \partial_{i}$ be in $\mathbb{D}_{1}$. For a generic function $\psi: \mathbb{R}^{d} \rightarrow \mathbb{R}$, denote by $\Psi(x, y):=\psi((x-y) / 2)$, and let $V_{x}\left(\right.$ resp. $\left.V_{y}\right)$ be a shorthand for $V \otimes \mathrm{id},($ resp. id $\otimes V)$. For each $k=1,2,3$ and $\psi \in W^{3, \infty}$ with compact support in the unit ball $B_{1} \subset \mathbb{R}^{d}$, it holds uniformly in $\epsilon \in(0,1]$ :

$$
\left|\left(\nabla_{ \pm}\right)^{k-1} \circ T_{\epsilon}^{-1} \circ\left(V_{x}+V_{y}\right) \circ T_{\epsilon}[\Psi(x, y)]\right| \leq|\sigma|_{W^{k, \infty}}|\psi|_{W^{k, \infty}} .
$$

for a.e. $(x, y) \in \mathbb{R}^{d} \times \mathbb{R}^{d}$.

Proof of the Theorem Step 1: the key estimate. We first show that for $\Phi \in W_{0}^{k, \infty}(\Omega)$, and with $V$ as in Lemma A.1:

$$
\left|\left(\nabla_{ \pm}\right)^{k-1}\left(T_{\epsilon}\right)^{-1}\left(V_{x}+V_{y}\right) T_{\epsilon} \Phi\right|_{L^{\infty}(\Omega)} \leq C|\sigma|_{W^{k, \infty}}|\Phi|_{W_{0}^{k, \infty}(\Omega)} .
$$

By density, it will be enough to show (A.9) on functions of the form $\Phi(x, y)=$ $\phi\left(\frac{x+y}{2}\right) \psi\left(\frac{x-y}{2}\right)$, with $\psi$ compactly supported in $B_{1}$. For such $\Phi$, we have

$$
\begin{aligned}
& T_{\epsilon}^{-1}\left(V_{x}+V_{y}\right) T_{\epsilon} \Phi(x, y)=T_{\epsilon}^{-1}\left(V_{x}+V_{y}\right)\left[\phi\left(\frac{x+y}{2}\right)\right] \psi\left(\frac{x-y}{2 \epsilon}\right) \\
& +\phi\left(\frac{x+y}{2}\right) T_{\epsilon}^{-1}\left(V_{x}+V_{y}\right)\left[\psi\left(\frac{x-y}{2 \epsilon}\right)\right]=I_{\epsilon}+I I_{\epsilon} .
\end{aligned}
$$

Using the new coordinates, we have the following expression for the first term:

$$
I_{\epsilon}=\frac{1}{2}\left(\sigma\left(x_{+}+\epsilon x_{-}\right)+\sigma\left(x_{+}-\epsilon x_{-}\right)\right) \cdot \nabla \phi\left(x_{+}\right) \psi\left(x_{-}\right) .
$$

By the commutation relations

$$
\nabla_{+} T_{\epsilon}=T_{\epsilon} \nabla_{+}, \quad \text { and } \quad \nabla_{-} T_{\epsilon}=\epsilon^{-1} T_{\epsilon} \nabla_{-} .
$$

it is then easily seen (see [38, Proposition 6.1] for details) that for $k=1,2,3$ :

$$
\operatorname{ess} \sup _{x_{+}, x_{-}}\left|\left(\nabla_{ \pm}\right)^{k-1} I_{\epsilon}\right| \leq|\sigma|_{W^{k, \infty}}|\Phi|_{W^{k, \infty}} \leq C \omega_{B}(s, t)^{i \alpha}|\Phi|_{W^{k, \infty}}
$$

For the second term, we can use Lemma A.1, since by assumption $\psi$ is supported on the unit ball of $\mathbb{R}^{d}$. We have

$$
\operatorname{ess} \sup _{x_{+}, x_{-}}\left|\left(\nabla_{ \pm}\right)^{k-1}\left[I I_{\epsilon}\right]\right| \leq|\sigma|_{W^{k}}|\Phi|_{W^{k, \infty}} .
$$

Step 2: uniform estimates on the first component. For $V \in \mathbb{D}_{1}$ define

$$
\mathbb{\Gamma}(V):=V \otimes \mathrm{id}+\mathrm{id} \otimes V,
$$

and further let

$$
\mathbb{\Gamma}^{\epsilon}(V):=T_{\epsilon}^{*} \mathbb{\Gamma}(V)\left(T_{\epsilon}^{*}\right)^{-1}
$$


Particularizing (A.9) with $V=B_{s t}^{1} \in \mathbb{D}_{1}$ for fixed $s, t$, we see by definition of $\Gamma_{s t}^{1, \epsilon}(B)$ that

$$
\begin{aligned}
\left|\Gamma_{s t}^{1, \epsilon}(\mathbf{B})\right|_{\mathscr{L}\left(W^{-k+1,1}(\Omega), W^{-k, 1}(\Omega)\right)} \equiv\left|\Gamma^{\epsilon}\left(B_{s t}^{1}\right)\right|_{\mathscr{L}\left(W^{-k+1,1}(\Omega), W^{-k, 1}(\Omega)\right)} \\
\leq\left|\Gamma^{\epsilon}\left(B_{s t}^{1}\right)^{*}\right|_{\mathscr{L}\left(W_{0}^{k, \infty}(\Omega), W_{0}^{k-1, \infty}(\Omega)\right)} \\
\equiv\left|T_{\epsilon}^{-1}\left(B_{x, s t}^{1, *}+B_{y, s t}^{1, *}\right) T_{\epsilon}\right|_{\mathscr{L}\left(W_{0}^{k, \infty}(\Omega), W_{0}^{k-1, \infty}(\Omega)\right)} \\
\leq C \omega_{B}(s, t)^{\alpha},
\end{aligned}
$$

for any $k \in\{1,2,3\}$. This yields the first part of the claimed estimate.

Note that, since the bracket $[\mathbf{B}]_{s t}$ has order one (B is geometric), we can let $V=[\mathbf{B}]_{s t}$ in the previous computations in order to obtain

$$
\left|\mathbb{\Gamma}^{\epsilon}\left([\mathbf{B}]_{s t}\right)\right|_{\mathscr{L}\left(W^{-k+1,1}(\Omega), W^{-k, 1}(\Omega)\right)} \leq C \omega_{B}(s, t)^{2 \alpha} .
$$

Step 3: uniform estimates on the second component. Recalling that $[\mathbf{B}]:=B^{2}-B^{1} \circ B^{1} / 2$, we have by definition of $\Gamma_{s t}^{2}(B)$ :

$$
\begin{aligned}
\Gamma_{s t}^{2, \epsilon}(\mathbf{B}) & =T_{\epsilon}^{-1}\left(B_{x}^{2}+B_{x}^{1} B_{y}^{1}+B_{y}^{2}\right)_{s t} T_{\epsilon} \\
& \equiv T_{\epsilon}^{-1}\left(\frac{1}{2} B_{x}^{1} B_{x}^{1}+[\mathbf{B}]_{x}+B_{x}^{1} B_{y}^{1}+\frac{1}{2} B_{y}^{1} B_{y}^{1}+[\mathbf{B}]_{y}\right)_{s t} T_{\epsilon} \\
& =T_{\epsilon}^{-1}\left(\frac{1}{2}\left(B_{x}^{1}+B_{y}^{1}\right)^{2}+[\mathbf{B}]_{x}+[\mathbf{B}]_{y}\right)_{s t} T_{\epsilon} .
\end{aligned}
$$

Otherwise said, we have the algebraic identity $\Gamma_{s t}^{2, \epsilon}(\mathbf{B})=\frac{1}{2} \Gamma_{s t}^{1, \epsilon}(\mathbf{B}) \circ \Gamma_{s t}^{1, \epsilon}(\mathbf{B})+\mathscr{B}_{s t}^{\epsilon}, \quad$ where $\mathscr{B}_{s t}^{\epsilon}:=T_{\epsilon}^{-1}\left([\mathbf{B}]_{x}+[\mathbf{B}]_{y}\right)_{s t} T_{\epsilon}$.

But if $k \in\{-1,0\}$, the estimate (A.13) shows that

$$
\left|\mathscr{B}_{s t}^{\epsilon}\right|_{\mathscr{L}\left(W^{k, 1}, W^{k-1,1}\right)} \leq C \omega_{B}(s, t)^{2 \alpha} .
$$

We can now conclude thanks to (A.15) and Step 2, since for $k=0,-1$ :

$$
\begin{aligned}
\left|\Gamma_{s t}^{2, \epsilon}(\mathbf{B})\right|_{\mathscr{L}\left(W^{k, 1}, W^{k-2,1}\right) \leq} & \frac{1}{2}\left|\Gamma_{s t}^{1, \epsilon}(\mathbf{B})\right|_{\mathscr{L}\left(W^{k, 1}, W^{k-1,1}\right)}\left|\Gamma_{s t}^{1, \epsilon}(\mathbf{B})\right|_{\mathscr{L}\left(W^{k-1,1}, W^{k-2,1}\right)} \\
& +\left|\mathscr{B}_{s t}^{\epsilon}\right|_{\mathscr{L}\left(W^{k, 1}, W^{k-2,1}\right)} \leq C \omega_{B}(s, t)^{2 \alpha},
\end{aligned}
$$

which finishes the proof of Theorem 4.1.

\section{Appendix B: Further remarks and comments}

\section{B.1 Uniqueness of the Gubinelli derivative}

Let $u$ be such that

$$
\mathrm{d} u=f \mathrm{~d} t+\mathrm{d} \mathbf{B}\left(g, g^{\prime}\right),
$$


where $f \in L\left(0, T ; W^{-1, p}\right)$ while $\left(g, g^{\prime}\right) \in \mathcal{D}_{B}^{\alpha, p}$, and write

$$
u \simeq\left(f ; g, g^{\prime}\right) \text {. }
$$

It is natural to ask under which condition one can have uniqueness of the triple $\left(f ; g, g^{\prime}\right)$ such that $u \simeq\left(f ; g, g^{\prime}\right)$, a question that relates the Doob-Meyer decomposition for semimartingales. Such uniqueness is certainly not true in general because our definition of a differential rough driver could accomodate that of $\dot{B}:=\dot{Z} \partial_{x}$, where $Z \in C^{\infty}(0, T ; \mathbb{R})$. Indeed, in this case one can arbitrarily choose $g^{\prime}=0$ for any $u$ and alternatively represent the element $u \simeq(f ; g, 0)$ by writing instead $u \simeq\left(f+\dot{Z} \partial_{x} g ; 0,0\right)$.

In the finite-dimensional case however (for instance replacing $B$ by a path $Z$ of $\frac{1}{\alpha}$-finite variation with values in $\mathbb{R}$ ), the decomposition (3.12) is indeed unique in the case where $Z$ is truly rough [25], i.e. when there exists a dense set of times $t \in[0, T]$ such that

$$
\limsup _{s \rightarrow t} \frac{\left|Z_{s t}\right|}{\omega_{Z}(s, t)^{2 \alpha}}=\infty \text {. }
$$

The situation here is different in the sense that assuming $B=Z \sigma \cdot \nabla$ with $Z$ as in (B.1) does not guarantee uniqueness of the couple $(f, g)$ in (3.12). To wit, assume that $d=2$, and let $B$ as above with $\sigma=(0,1)$. If $(f, g)$ satisfy (3.12), then it is immediately seen that any path of the form $t \mapsto g_{t}(x, y)+\tilde{g}_{t}(x)$ where $\tilde{g} \in \mathcal{V}_{1}^{\alpha}\left(0, T ; L^{2}(\mathbb{R})\right)$ is a function of the first variable only, will also satisfy (3.12). In this counterexample, one sees that the space variable plays an important role in the discussion, and that if one aims at the uniqueness of the above decomposition, then some "non-degeneracy" assumptions on the differential operator $\sigma \cdot \nabla$ are in order. Let us now formulate a natural sufficient condition under which uniqueness of the Gubinelli derivative holds.

Assume that we are given a family $B_{t}$ of (non-necessarily differential) operators such that the mapping $[0, T] \rightarrow \cap_{-2 \leq k \leq 0} \mathscr{L}\left(H^{k}, H^{k-1}\right), t \mapsto B_{t}$ is $\alpha$-Hölder continuous, where as before $\alpha>1 / 3$. For notational simplicity, we denote in the sequel $B_{s t}:=\delta B_{s t}$.

Theorem B.1 Assume the existence of $\gamma \in\left[\alpha, \frac{3}{2} \alpha\right)$, such that the following ellipticity condition is satisfied: there is a constant $\Lambda>0$, such that for every $\varphi$ in $H^{-1}$, and for each $(s, t) \in \Delta \cap D^{2}$,

$$
\left|B_{s t} \varphi\right|_{H^{-2}} \geq \Lambda(t-s)^{\gamma}|\varphi|_{H^{-1}}
$$

where we are given some dense subset $D$ of $[0, T]$.

Let $u \in L^{\infty}\left(0, T ; L^{2}\right) \cap C^{\alpha}\left(0, T ; H^{-1}\right)$ and suppose that $g, \tilde{g} \in C^{\alpha}\left(0, T ; H^{-1}\right)$ are both Gubinelli derivatives for $u$ in the Hölder sense, by which we mean that

$$
\sup _{0 \leq s<t \leq T} \frac{\left|R_{s t}^{g}\right|_{H^{-2}}}{(t-s)^{2 \alpha}}=\sup _{0 \leq s<t \leq T} \frac{\left|\delta u_{s t}-B_{s t} g_{s}\right|_{H^{-2}}}{(t-s)^{2 \alpha}}<\infty,
$$

and similar for $\tilde{g}$. Then, $g=\tilde{g}$.

Proof Fix $(s, t) \in \Delta \cap D^{2}$. The assumption (B.2) implies that the bilinear form

$$
a_{s t}: H^{-1} \times H^{-1} \rightarrow \mathbb{R}, \quad a_{s t}(u, v):=\left(B_{s t} u, B_{s t} v\right)_{H^{-2}}
$$

is $H^{-1}$-coercive. Therefore, if $F: H^{-1} \rightarrow \mathbb{R}$ is linear and continuous, the variational problem

admits a unique solution

$$
\left\{\begin{array}{l}
\text { Find } u \in V:=H^{-1} \text { such that } \\
\forall v \in V, \quad a_{s t}(u, v)=F(v) .
\end{array}\right.
$$

$$
u=\mathbf{T}_{s t} F \in H^{-1} .
$$


Moreover, it is easily seen that the Riesz isomorphism between $H^{-2}$ and its dual identifies the dual of $H^{-1}$ with $H^{-3}$, hence the operator norm of $\mathbf{T}_{s t}:\left(H^{-1}\right)^{*} \simeq H^{-3} \rightarrow H^{-1}$ is estimated above as

$$
\left|\mathbf{T}_{s t}\right|_{\mathscr{L}\left(H^{-3}, H^{-1}\right)} \leq \Lambda^{-2}(t-s)^{-2 \gamma} .
$$

Furthermore, if $B_{s t}^{\dagger}$ denotes the adjoint of $B_{s t}$ with respect to the $H^{-2}$-inner product, observe thanks to (B.3) that $\mathbf{T}_{s t}$ is the inverse transform of

$$
B_{s t}^{\dagger} \circ B_{s t}: H^{-1} \rightarrow H^{-3} \text {. }
$$

Let $g$ be a Gubinelli derivative for $u$. From the above discussion, one infers the relation

$$
g_{s}=\mathbf{T}_{s t} B_{s t}^{\dagger} \delta u_{s t}-\mathbf{T}_{s t} B_{s t}^{\dagger} R_{s t}^{u}=: I+I I .
$$

By assumption on $R_{s t}^{u}:=\delta u_{s t}-B_{s t} g_{s}$, it holds

$$
|I I|_{H^{-1}} \leq \Lambda^{-2}(t-s)^{-2 \gamma}\left|B_{s t}^{\dagger} R_{s t}^{u}\right|_{H^{-3}} \leq \Lambda^{-2}(t-s)^{3 \alpha-2 \gamma}\left\|R^{u}\right\|_{C_{2}^{\alpha}\left(H^{-2}\right)} .
$$

Hence, letting $t_{n} \searrow s, t_{n} \in D$, one sees that

$$
|I I|_{L^{2}} \leq C\left(t_{n}-s\right)^{3\left(\alpha-\frac{2}{3} \gamma\right)} \rightarrow 0 \quad \text { as } n \rightarrow \infty .
$$

This implies that $g_{t}$ is uniquely determined by the relation

$$
g_{t}=\lim _{s \rightarrow t, s \in D} \mathbf{T}_{s t} B_{s t}^{\dagger} \delta u_{s t} \text { in } H^{-1}
$$

thus proving our claim.

Example B.1 Let $d=1$, and consider a 1-dimensional, $\alpha$-Hölder rough path $\left(Z^{1}, Z^{2}\right) \in$ $\mathscr{C}^{\alpha}(0, T ; \mathbb{R})$ such that for some $D$ as above it holds

$$
\left|Z_{s t}\right| \geq c(t-s)^{\gamma}, \quad \text { for every }(s, t) \in \Delta \cap D^{2},
$$

where we are given some constant $\gamma \in[\alpha, 2 \alpha$ ) (this implies in particular true roughness for $Z$, in the sense of (B.1)). Moreover, let $\sigma \in W^{3, \infty}$ be bounded below, namely such that there exist constants $\underline{\sigma}>0$ with the property that $\sigma(x) \geq \underline{\sigma}$, for almost every $x \in \mathbb{R}^{d}$.

Then, it is easily seen that (B.2) holds with the differential rough driver B given by Example 2.1 with $\rho=0$, where $\Lambda=\Lambda(c, \underline{\sigma})>0$.

\section{B.2 Brackets}

For a geometric rough path $\left(Z^{1, \mu}, Z^{2, \mu \nu}\right)_{1 \leq \mu, v \leq m}$ it is well-known that the symmetric part of $Z^{2}$ is expressed in terms of $Z^{1}$, as follows

$$
\operatorname{sym} Z_{s t}^{2, \mu \nu} \equiv \frac{Z_{s t}^{2, \mu \nu}+Z_{s t}^{2, v \mu}}{2}=\frac{Z_{s t}^{1, v} Z_{s t}^{1, \mu}}{2}, \quad \text { for all } 1 \leq \mu, v \leq m,
$$

and every $(s, t) \in \Delta$ (see [50]). Alternatively, this means that the bracket $[Z]_{s t}:=\operatorname{sym} Z_{s t}^{2}-$ $\frac{1}{2}\left(Z_{s t}^{1}\right)^{2}$ vanishes for geometric rough paths. By analogy, in the case of an differential rough driver $\mathbf{B}$, we introduced the bracket as the following family of differential operators:

$$
[\mathbf{B}]_{s t}:=B_{s t}^{2}-\frac{1}{2} B_{s t}^{1} \circ B_{s t}^{1}, \quad(s, t) \in \Delta,
$$

(see Lemma 2.1). In contrast with what is encountered in the classical theory, note that the bracket does not vanish in general for $\mathbf{B}$ geometric, which is a side effect of the noncommutativity of the algebra of differential operators. Nevertheless, we saw in Lemma 2.1 that, as a consequence of geometricity, $[\mathbf{B}]$ takes values in the space of $\mathbb{D}_{1}$. In particular, 
unless $B_{s t}^{1} \in \mathbb{D}_{0}$, we see that a a cancellation occurs, since in that case $[\mathbf{B}]_{s t}$ has stricly lower order than $B_{s t}^{2}$. This can be seen as a non-commutative counterpart of the fact that the bracket of geometric rough paths is zero.

Remark B.1 If B denotes a differential rough driver, then by definition of the bracket [B] in (B.5), we have

$$
B_{s t}^{2}(\phi \psi)=\left(B_{s t}^{2} \phi\right) \psi+\left(B_{s t}^{1} \phi\right)\left(B_{s t}^{1} \psi\right)+\phi\left(B_{s t}^{2} \psi\right)-\mathfrak{l}_{s t}(\phi, \psi)
$$

where $\mathfrak{l}_{s t}$ denotes the (generally unbounded) bilinear operator

$$
\phi, \psi \mapsto \mathfrak{l}_{s t}(\phi, \psi)=[\mathbf{B}]_{s t}(\phi \psi)-\left([\mathbf{B}]_{s t} \phi\right) \psi-\phi\left([\mathbf{B}]_{s t} \psi\right) .
$$

To give a concrete example, consider a filtered probability space $\left(\Omega, \mathcal{A}, \mathbb{P},\left\{\mathcal{F}_{t}\right\}_{t \in[0, T]}\right)$, let $W: \Omega \times[0, T] \rightarrow \mathbb{R}$ be a Brownian motion, and fix $V \in \mathbb{D}_{1} \backslash \mathbb{D}_{0}$. Define the (random) differential rough driver $\mathbf{B}^{\text {Itô }}(\omega)$ by $B_{s t}^{\text {Itô, } 1}:=\left(W_{t}-W_{s}\right) V$ and, observing that $\mathbb{P}$-a.s., $\int_{s}^{t}\left(W_{r}-W_{s}\right) \mathrm{d} W_{r}=\frac{1}{2}\left[\left(W_{t}-W_{s}\right)^{2}-(t-s)\right]$ (Itô sense), let

$$
B_{s t}^{\mathrm{It} \hat{\mathrm{t}}, 2}:=\frac{1}{2}\left[\left(W_{t}-W_{s}\right)^{2}-(t-s)\right] V^{2} .
$$

With this definition, we have

$$
\left[\mathbf{B}^{\mathrm{It} \hat{0}}\right]_{s t}=-\frac{(t-s)}{2} V^{2},
$$

showing that $[\mathbf{B}] \in \mathbb{D}_{2} \backslash \mathbb{D}_{1}$, almost surely.

Remark B.2 As seen in the above remark, if B is not geometric, its bracket [B] (see (B.5)) is generally not first order. In the stochastic context, this has to do with the violation of stochastic parabolicity assumption, as can be seen as follows. Using the notations of Remark (B.1), we see that in the proof of the product formula, the (4.34) must be changed to

$$
\begin{aligned}
\lim _{\epsilon \rightarrow 0}\left\langle\Gamma_{s t}^{2, \epsilon}(\mathbf{B})(u \otimes v)_{s}^{\epsilon}, \Phi\right\rangle & =\left\langle\left(B_{s t}^{2} u_{s}\right) v_{s}+\left(B_{s t}^{1} u_{s}\right)\left(B_{s t}^{1} v_{s}\right)+u_{s}\left(B_{s t}^{2} v_{s}\right), \phi\right\rangle \\
& \equiv\left\langle B_{s t}^{2}\left(u_{s} v_{s}\right), \phi\right\rangle+\left\langle\mathfrak{l}_{s t}\left(u_{s}, v_{s}\right), \phi\right\rangle .
\end{aligned}
$$

If we let furthermore $u=v$ where $u$ is an $L^{2}$-energy solution of (2.24), $\mathbf{B}=\mathbf{B}^{\mathrm{It} \hat{\mathrm{o}}}$, and $\phi=1$, we have

$$
\left\langle\mathfrak{l}_{s t}\left(u_{s}, u_{s}\right), 1\right\rangle=(t-s) \int_{U}\left(V u_{s}\right)^{2} \mathrm{~d} x .
$$

The latter competes with the term $-2 \lambda \iint_{[s, t] \times U}|\nabla u|^{2} \mathrm{~d} x \mathrm{~d} r$, which is brought by the elliptic part of the equation. In particular, the usual technique to obtain the energy estimate on $u$ fails, unless the coefficients of $V$ are taken small with respect to $\lambda$. This illustrates the importance of the geometricity assumption in our results.

\section{References}

1. Albiac, F., Kalton, N.J.: Topics in Banach space theory, vol. 233, Springer (2016)

2. Ambrosio, L.: Transport equation and Cauchy problem for BV vector fields. Inventiones Mathematicae 158(2), 227-260 (2004)

3. Bailleul, I., Bernicot, F.: Higher order paracontrolled calculus. Forum of Mathematics, Sigma. Vol. 7. Cambridge University Press (2019)

4. Bailleul, I., Gubinelli, M.: Unbounded rough drivers. Annales Mathématiques de la Faculté des Sciences de Toulouse 26(4), 795-830 (2017) 
5. Bailleul, I., Riedel, S.: Rough flows. Journal of the Mathematical Society of Japan (2019)

6. Bellingeri, C.C.: An Itô type formula for the additive stochastic heat equation. Electronic Journal of Probability 25 (2020)

7. Bogachev, V.I.: Measure theory, vol. 1, Springer Science \& Business Media (2007)

8. Brezis, H.: Functional analysis, Sobolev spaces and partial differential equations. Springer Science \& Business Media (2010)

9. Cannizzaro, G., Chouk, K.: Multidimensional SDEs with singular drift and universal construction of the polymer measure with white noise potential. The Annals of Probability 46(3), 1710-1763 (2018)

10. Caruana, M., Friz, P.K.: Partial differential equations driven by rough paths. Journal of Differential Equations 247(1), 140-173 (2009)

11. Caruana, M., Friz, P.K., Oberhauser, H.: A (Rough) Pathwise Approach to a Class of Non-Linear Stochastic Partial Differential Equations. In: Annales De L'institut Henri Poincaré (C) Non Linear Analysis, vol. 28, pp. 27-46. Elsevier, Amsterdam (2011)

12. Catellier, R.: Rough linear transport equation with an irregular drift. Stochastics and Partial Differential Equations: Analysis and Computations 4(3), 477-534 (2016)

13. Coutin, L., Lejay, A.: Perturbed linear rough differential equations [Équations Différentielles Linéaires Rugueuses Perturbées]. Annales Mathématiques Blaise Pascal 21, 103-150 (2014)

14. Davie, A.M.: Differential Equations Driven by Rough Paths: an Approach via Discrete Approximation. In: Applied Mathematical Research Express. Citeseer (2007)

15. Delarue, F., Diel, R.: Rough paths and 1d SDE with a time dependent distributional drift: application to polymers. Probab. Theory Relat. Fields 165(1-2), 1-63 (2016)

16. De Lellis, C.: Ordinary differential equations with rough coefficients and the renormalization theorem of Ambrosio. Séminaire Bourbaki 972, 2006-2007 (2007)

17. Diehl, J., Friz, P.K., Stannat, W.: Stochastic partial differential equations: a rough path view. Annales Mathématiques de la Faculté des Sciences de Toulouse, 26(4) (2017)

18. Deya, A., Gubinelli, M., Tindel, S.: Non-linear rough heat equations. Probab. Theory Relat. Fields 153(12), 97-147 (2012)

19. Deya, A., Gubinelli, M., Hofmanová, M., Tindel, S.: A priori estimates for rough PDEs with application to rough conservation laws. J. Funct. Anal. 276(12), 3577-3645 (2019)

20. DiPerna, R.J., Lions, P.L.: Ordinary differential equations, transport theory and Sobolev spaces. Inventiones Mathematicae 98(3), 511-547 (1989)

21. Feyel, D., de La Pradelle, A., Mokobodzki, G.: A non-commutative sewing lemma. Electron. Commun. Probab. 13, 24-34 (2008)

22. Flandoli, F., Gubinelli, M., Priola, E.: Well-posedness of the transport equation by stochastic perturbation. Inventiones Mathematicae 180(1), 1-53 (2010)

23. Friz, P.K., Hairer, M.: A course on rough paths: with an introduction to regularity structures. Springer (2014)

24. Friz, P.K., Oberhauser, H.: On the splitting-up method for rough (partial) differential equations. Journal of Differential Equations 251(2), 316-338 (2011)

25. Friz, P.K., Shekkar, A.: Doob-meyer for rough paths. Special Varadhan issue of Bulletin of Institute of Mathematics Academia Sinica New Series (2012)

26. Friz, P.K., Oberhauser, H.: Rough path stability of (semi-) linear SPDEs. Probab. Theory Relat. Fields 158(1-2), 401-434 (2014)

27. Friz, P.K., Victoir, N.B.: Multidimensional Stochastic Processes as Rough Paths: Theory and Applications, vol. 120. Cambridge University Press, Cambridge (2010)

28. Gerasimovics, A., Hairer, M.: Hörmander's theorem for semilinear SPDEs. Electronic Journal of Probability 24 (2019)

29. Gerasimovics, A., Hocquet, A., Nilssen, T.: Non-autonomous rough semilinear PDEs and the multiplicative Sewing Lemma. arXiv preprint arXiv:1907.1339

30. Gubinelli, M., Imkeller, P., Perkowski, N.: Paracontrolled Distributions and Singular PDEs. In: Forum of Mathematics, Pi, vol. 3, p. e6. Cambridge Univ. Press, Cambridge (2015)

31. Gubinelli, M., Tindel, S.: Rough evolution equations. The Annals of Probability 38(1), 1-75 (2010)

32. Gubinelli, M.: Controlling rough paths. J. Funct. Anal. 216, 86-140 (2004)

33. Gess, B.: Regularization and well-posedness by noise for ordinary and partial differential equations. In: International Conference on Stochastic Partial Differential Equations and Related Fields, pp. 43-67. Springer, Cham (2016)

34. Gyöngy, I., Shmatkov, A.: Rate of convergence of Wong-Zakai approximations for stochastic partial differential equations. Appl. Math. Optim. 54(3), 341-341 (2006)

35. Hairer, M.: Solving the KPZ equation. Annals of Mathematics, pp. 559-664 (2013)

36. Hairer, M.: A theory of regularity structures. Inventiones Mathematicae 198(2), 269-504 (2014) 
37. Hairer, M., Weber, H.: Rough Burgers-like equations with multiplicative noise. Probab. Theory Relat. Fields 155(1-2), 71-126 (2013)

38. Hocquet, A., Hofmanová, M.: An energy method for rough partial differential equations. Journal of Differential Equations 265(4), 1407-1466 (2018)

39. Hofmanová, M., Leahy, J.M., Nilssen, T.: On the Navier-Stokes equation perturbed by rough transport noise. J. Evol. Equ. 19(1), 203-247 (2019)

40. Kim, K.-H.: On $l_{p}$-theory of stochastic partial differential equations of divergence form in $c^{1}$ domains. Probability Theory and Related Fields 130(4), 473-492 (2004)

41. Kotelenez, P.M., Kurtz, T.G.: Macroscopic limits for stochastic partial differential equations of McKeanVlasov type. Probab. Theory Relat. Fields 146(1-2), 189 (2010)

42. Krylov, N.V.: An analytic approach to SPDEs. Stochastic Partial Differential Equations: Six Perspectives 64, 185-242 (1999)

43. Krylov, N.V.: On the itô-Wentzell formula for distribution-valued processes and related topics. Probab. Theory Relat. Fields 150(1-2), 295-319 (2011)

44. Krylov, N.V.: A relatively short proof of itô's formula for SPDEs and its applications. Stochastic Partial Differential Equations: Analysis and Computations 1(1), 152-174 (2013)

45. Krylov, N.V., Rozovskii, B.L.: Stochastic evolution equations. Journal of Soviet Mathematics 16(4), 1233-1277 (1981)

46. Ladyzhenskaya, O., Solonnikov, V., Uraltseva, N.: Linear and quasilinear parabolic equations of second order. Translation of Mathematical Monographs, AMS Rhode Island (1968)

47. Lejay, A.: An Introduction to Rough Paths. In: Séminaire De ProbabilitéS XXXVII, pp. 1-59. Springer, Berlin (2003)

48. Lions, P.L., Souganidis, P.E.: Fully nonlinear stochastic partial differential equations. Comptes Rendus de l'Académie des Sciences-Series I-Mathematics 326(9), 1085-1092 (1998)

49. Lions, P.L., Souganidis, P.E.: Fully nonlinear stochastic partial differential equations: non-smooth equations and applications. Comptes Rendus de l'Académie des Sciences-Series I-Mathematics 327(8), 735-741 (1998)

50. Lyons, T.J.: Differential equations driven by rough signals. Revista Matemática Iberoamericana 14(2), 215-310 (1998)

51. Lyons, T.J., Qian, Z., Qian, Z.: System control and rough paths. Oxford University Press (2002)

52. Mohammed, S.E.A., Nilssen, T.K., Proske, F.N.: Sobolev differentiable stochastic flows for SDEs with singular coefficients. Applications to the Transport Equation. The Annals of Probability 43(3), 1535$1576(2015)$

53. Moser, J.: A Harnack inequality for parabolic differential equations. Communications on Pure and Applied Mathematics 17(1), 101-134 (1964)

54. Nilssen, T.: Rough linear PDE's with discontinuous coefficients - existence of solutions via regularization by fractional Brownian motion. Electronic Journal of Probability 25 (2020)

55. Otto, F., Weber, H.: Quasilinear SPDEs via rough paths. Arch. Ration. Mech. Anal. 232(2), 873-950 (2019)

56. Pardoux, E.: Stochastic partial differential equations and filtering of diffusion processes. Stochastics 3(1-4), 127-167 (1980)

57. Reed, M., Simon, B.: Methods of modern mathematical physics, volume 1 Functional analysis Academic (1980)

58. Revuz, D., Yor, M.: Continuous martingales and Brownian motion, vol. 293, (3rd edn) Springer (1999)

59. Young, L.C.: An inequality of the hölder type, connected with Stieltjes integration. Acta Mathematica 67(1), 251-282 (1936)

60. Zambotti, L., di Milano, P.: Itô-tanaka's formula for stochastic partial differential equations driven by additive space-time white noise. Stochastic Partial Differential Equations and Applications-VII 245, 337-347 (2006)

Publisher's Note Springer Nature remains neutral with regard to jurisdictional claims in published maps and institutional affiliations. 